\title{
The Impact of COVID-19 on Kidney Transplant Recipients in Pre-Vaccination and Delta Strain Era: A Systematic Review and Meta-Analysis
}

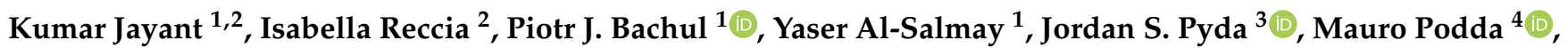 \\ Angelica Perez-Gutierrez ${ }^{1}$, Frank J. M. F. Dor ${ }^{5}$, Yolanda Becker ${ }^{1}$, Diego di Sabato ${ }^{1}$, John LaMattina ${ }^{1}$, Rolf Barth ${ }^{1}$, \\ John Fung ${ }^{1}$ and Piotr Witkowski ${ }^{1, *}$
}

Citation: Jayant, K.; Reccia, I.; Bachul, P.J.; Al-Salmay, Y.; Pyda, J.S.; Podda, M.; Perez-Gutierrez, A.; Dor, F.J.M.F.; Becker, Y.; di Sabato, D.; et al. The Impact of COVID-19 on Kidney Transplant Recipients in

Pre-Vaccination and Delta Strain Era: A Systematic Review and Meta-Analysis. J. Clin. Med. 2021, 10, 4533. https://doi.org/10.3390/ jcm10194533

Academic Editor: Hirofumi Noguchi

Received: 15 August 2021

Accepted: 25 September 2021

Published: 30 September 2021

Publisher's Note: MDPI stays neutral with regard to jurisdictional claims in published maps and institutional affiliations.

Copyright: (c) 2021 by the authors. Licensee MDPI, Basel, Switzerland. This article is an open access article distributed under the terms and conditions of the Creative Commons Attribution (CC BY) license (https:/ / creativecommons.org/licenses/by/ $4.0 /)$.
1 Transplantation Institute, Department of Surgery, University of Chicago, Chicago, IL 60637, USA; Kumar.Jayant@uchospitals.edu (K.J.); Piotr.Bachul@uchospitals.edu (P.J.B.); Yaser.Al-Salmay@uchospitals.edu (Y.A.-S.); rperezgutierrez@bsd.uchicago.edu (A.P.-G.); ybecker@surgery.bsd.uchicago.edu (Y.B.); ddisabato@surgery.bsd.uchicago.edu (D.d.S.); jlamatti@bsd.uchicago.edu (J.L.); rbarth@bsd.uchicago.edu (R.B.); jfung@surgery.bsd.uchicago.edu (J.F.)

2 Department of Surgery and Cancer, Imperial College Healthcare NHS Trust, London W12 0HS, UK; isabella.reccia@nhs.net

3 Department of Surgery, Beth Israel Deaconess Medical Center, Boston, MA 02215, USA; jordanpyda@gmail.com

4 Department of Emergency Surgery, Azienda Ospedaliero-Universitaria Di Cagliari, University Hospital Policlinico Duilio Casula, 09124 Cagliari, Italy; mauropodda@ymail.com

5 Imperial College Renal and Transplant Centre, Hammersmith Hospital, Imperial College Healthcare NHS Trust, London W12 0HS, UK; frank.dor@nhs.net

* Correspondence: pwitkowski@surgery.bsd.uchicago.edu; Tel.: +1-773-702-2447

Abstract: Herein, we performed a meta-analysis of published clinical outcomes of corona virus disease 2019 (COVID-19) in hospitalized kidney transplant recipients. A systematic database search was conducted between December 1, 2019 and April 20, 2020. We analyzed 48 studies comprising 3137 kidney transplant recipients with COVID-19. Fever (77\%), cough (65\%), dyspnea (48\%), and gastrointestinal symptoms $(28 \%)$ were predominant on hospital admission. The most common comorbidities were hypertension (83\%), diabetes mellitus (34\%), and cardiac disease $(23 \%)$. The pooled prevalence of acute respiratory distress syndrome and acute kidney injury were $58 \%$ and $48 \%$, respectively. Invasive ventilation and dialysis were required in $24 \%$ and $22 \%$ patients, respectively. In-hospital mortality rate was as high as $21 \%$, and increased to over $50 \%$ for patients in intensive care unit (ICU) or requiring invasive ventilation. Risk of mortality in patients with acute respiratory distress syndrome (ARDS), on mechanical ventilation, and ICU admission was increased: OR = 19.59, $\mathrm{OR}=3.80$, and $\mathrm{OR}=13.39$, respectively. Mortality risk in the elderly was $\mathrm{OR}=3.90$; however, no such association was observed in terms of time since transplantation and gender. Fever, cough, dyspnea, and gastrointestinal symptoms were common on admission for COVID-19 in kidney transplant patients. Mortality was as high as $20 \%$ and increased to over $50 \%$ in patients in ICU and required invasive ventilation.

Keywords: COVID-19; kidney transplantation; SARS-CoV-2

\section{Introduction}

The initial strain of coronavirus, severe acute respiratory syndrome coronavirus 2 (SARS-CoV-2), is highly transmissible and has led to a global pandemic afflicting around sixty-five million people in the first year. To date, the reported mortality rate is $2-6 \%$, but rises to as high as $26 \%$ among hospitalized patients and represents a significant health concern in the elderly and populations with underlying comorbidities [1-3]. The implications of immunosuppression in the context of both SARS-CoV-2 infection and potential donor 
transmission are still being explored [4,5]. This uncertainty contributed to the decline in the kidney transplantation services during the initial stage of pandemic due to unquantified but potential high risk of morbidity and mortality [6,7]. On the other hand, pre-COVID-19, chronic kidney disease patients on dialysis are particularly vulnerable with an approximately ten-fold higher risk of mortality than the general population $[8,9]$. Consequently, Boyarsky et al. [10] recently reported a 2.2-fold (95\% CI, 1.88-2.62) increase in mortality for patients in the United States on the kidney transplantation waitlist during the early part of the pandemic according to the Scientific Registry of Transplant Recipients data. Given the significant overall mortality benefit following kidney transplantation for patients with end stage renal disease (ESRD), there is unprecedented need and urgency to quantify the risk of SARS-CoV-2 infection in these patients before and after transplant [10]. The objective of this meta-analysis was to better understand the clinical course of COVID-19 in kidney transplant recipients and to identify factors associated with clinical outcomes and mortality. Furthermore, this systematic review focuses on the understanding of attributes influencing mortality following COVID-19 in kidney transplant recipients in contrast to general population and chronic kidney disease patients.

\section{Materials and Methods}

\subsection{Search Strategy}

We performed a systematic literature search of articles indexed in PubMed, EMBASE, MedRxiv, Cochrane, Crossref, Scopus, and clinical trial registries. The search strategy was based on recommendations from the Cochrane Handbook for Systematic Reviews of Intervention and reported according to the guidelines of meta-analysis of observational studies in epidemiology [11]. The following MeSH terms were used: "COVID-19" AND "kidney transplantation"; "Coronavirus" AND "kidney transplantation"; "COVID-19" AND "kidney transplantation" AND "mortality"; "COVID-19" AND "kidney transplantation" AND "clinical outcomes". We performed additional free texts searches including renal transplantation as applicable. The initial database search was performed on 10 November 2020. Additional studies were identified via a manual search of preprints, case reports, abstracts, bibliographies, and a citation list of relevant articles using the free search terms "2019 novel coronavirus", "SARS-CoV-2", and "2019-nCoV infection". The additional search was completed on 20 April 2020. This study was registered in PROSPERO, which is an international database of prospectively registered systematic reviews (CRD42020189637).

\subsection{Inclusion and Exclusion Criteria}

All observational studies available in the literature pertaining to COVID-19 and kidney transplantation were included. All other articles or publication types including editorials, letters, reviews, case reports, case series with less than five cases, and articles with duplicate data were excluded. The selected studies were reviewed and the following parameters were extracted: clinical presentation, severity of respiratory disease, hospital admission, rate of intensive care unit (ICU) admission, diagnosis of acute respiratory distress syndrome (ARDS), need for mechanical ventilation, presence of acute kidney injury (AKI), need for renal replacement therapy (RRT), changes in white cell count (WBC), laboratory evidence of inflammation (e.g., ESR, CRP), modifications to the immunosuppressive regimen, whether other treatment was administered, presence of graft rejection, and mortality. Furthermore, since older age, male gender, and impact of time since transplantation were evaluated to determine the mortality risk in regard to these factors [12,13]. In addition, mortality risk was computed following COVID-19 in a setting of chronic kidney disease (CKD) patients on waitlist/dialysis or both with respect to kidney transplant recipients. Preferred Reporting Items for Systematic Reviews and Meta-analyses (PRISMA) guidelines were used to complete the search and the article selection. Figure 1 demonstrates the PRISMA flowchart and identifies the number of search results, articles meeting criteria, and articles selected for data extraction. 


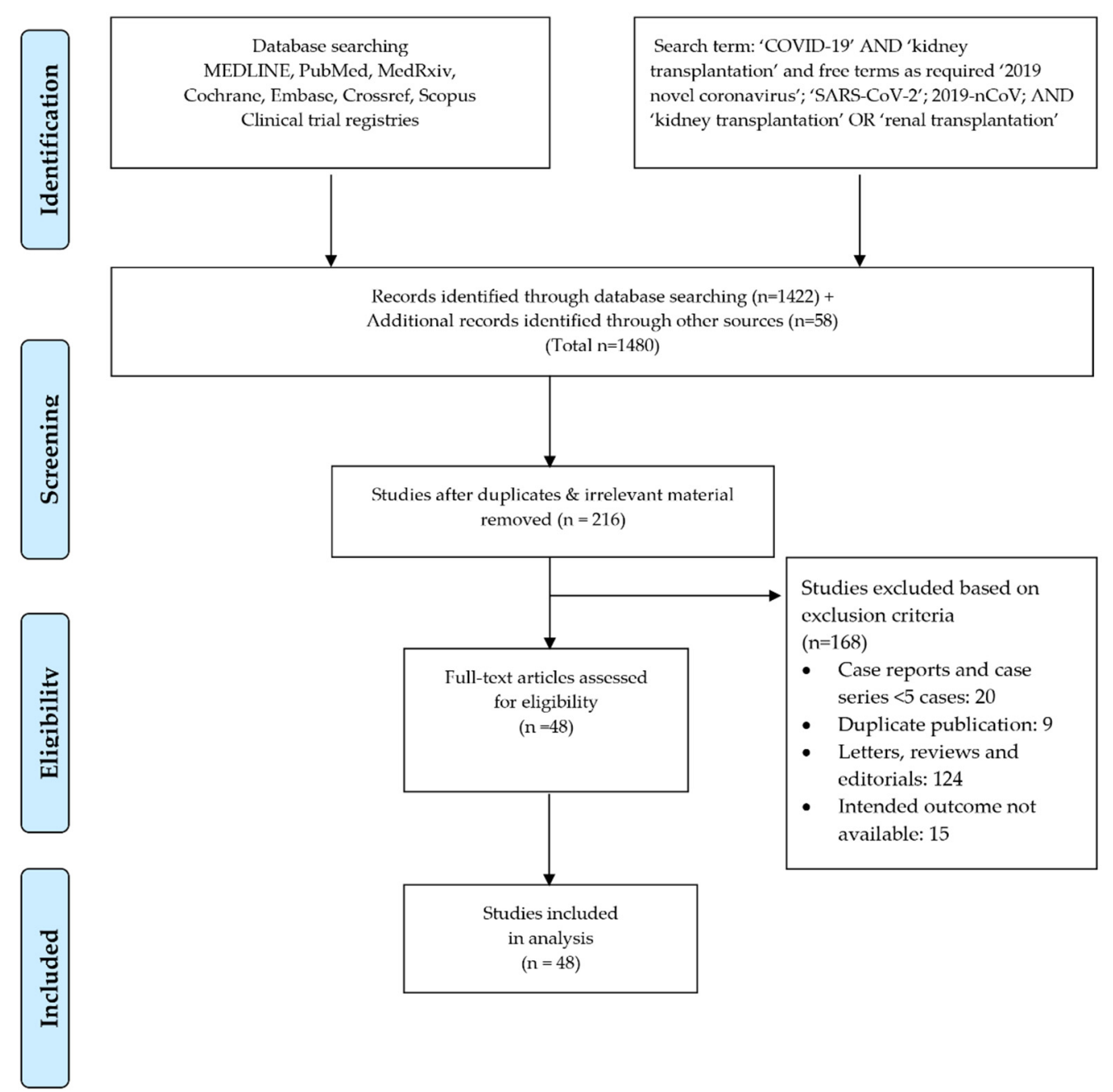

Figure 1. Search strategy and study selection used in this systematic review as per the PRISMA protocol.

\subsection{Data Extraction}

Two separate physician reviewers, KJ and IR, employed a two-stage method to independently screen the identified articles using a shared online form. During the initial review, the titles and abstracts were evaluated for the purpose of excluding ineligible articles. During the secondary review, the full or limited text (e.g., posters) were reviewed. In case of a discrepancy between the two initial reviewers, the items were discussed until a consensus was achieved with a third reviewer serving as the arbitrator (PW). Pre-defined data were extracted from the articles meeting criteria into a dataset and preliminary descriptive statistical analysis was performed to generate central tendency (i.e., mean or median) and dispersion (i.e., 95\% CI, IQR or range) for continuous variables. In scenarios where reported means and standard deviations of the variables of interest were not available (i.e., not reported in the identified articles), the values were inputted from the reported statistics (i.e., median, IQR or range) $[14,15]$. Heterogeneity among included studies was investigated using I2-statistics and classified in the following fashion: $\mathrm{I} 2$ of $\leq 25 \%$ was interpreted as low heterogeneity; I2 of $25-75 \%$ indicated moderate heterogeneity, and $\geq 75 \%$ was interpreted as higher heterogeneity [16]. Due to the heterogeneity of data within individual studies, and between different studies, the pooled prevalence of all attributes was calculated using the random-effect model in STATA/SE 16 (Stata, College Station, TX, USA). The risk of bias for observational studies was appraised through the quality assessment tool published by the National Institutes of Health (i.e., Quality Assessment 
Tool for Case Series Studies) [17], which is the preferred tool for assessments of risk of bias in systematic reviews registered in the PROSPERO protocols (Figure 2) [18].

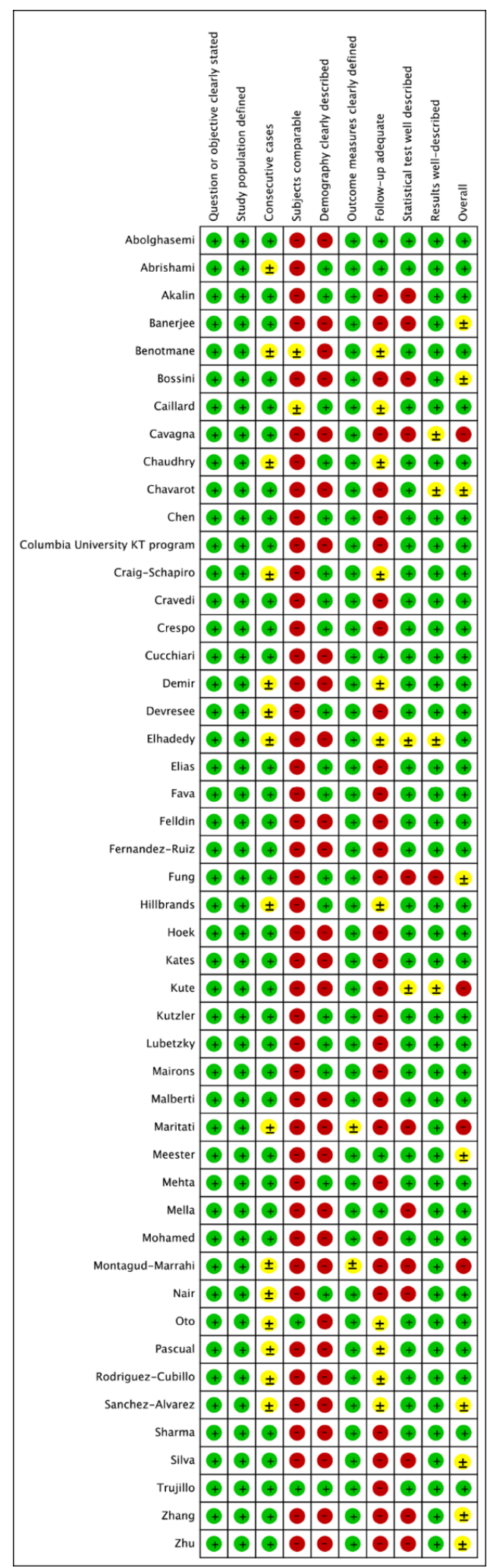

Figure 2. Quality assessment of included studies. (green-low risk of bias; yellow-unclear risk of bias; red-high risk of bias). 


\section{Results}

\subsection{Search Results}

The primary literature search yielded a total of 216 articles meeting the preliminary selection criteria. After careful evaluation, 168 articles were removed based on the exclusion criteria as detailed above. In the case of duplicate publications, the most recent available data were considered and included in the analysis. After the resolution of differences between reviewers and removal of duplicate reports, all available observational retrospective studies and case series were selected for data extraction [19-66]. This process identified 48 individual studies reporting a total of 3137 renal transplant patients with COVID-19 from 12 different countries located in North America, Europe, and Asia (Tables A1-A3). The detailed results of the meta-analysis are presented below.

\subsection{Demographic Data}

The pooled estimated mean age of the patients was 57.08 years (95\% CI $54.55-59.03$ years). The gender distribution in the reported data population was $66 \%$ (95\% CI 61-70\%) male. The post-transplant period was reported in 33 studies with a mean of 7.06 years ( $95 \%$ CI, 5.91-9.02 years). Of these, 17 studies provided sufficient data to assess the time between kidney transplantation and the diagnosis of COVID. Overall, $66.3 \%(827 / 1248)$ of COVID-19 positive patients were more than 1-2 years post kidney transplant and 33.7\% (421/696) were within 1-2 years of transplantation. Duration of COVID-19 symptoms before hospitalization was inconsistently reported and thus not analyzed.

\subsection{Comorbidities}

Meta-analysis utilizing a random-effects model was performed in order to identify the effect size (ES) of comorbidities in kidney transplant recipients with confirmed COVID-19 and expressed as a percentage. The estimated pooled prevalence of individual comorbidities is presented in Table A4. Type 2 diabetes mellitus (T2DM) was reported in 33 studies, in 621 out of 1785 patients with COVID-19 equating to a pooled prevalence of $34 \%$ (95\% CI, 29-40\%). Hypertension was determined from data available in 32 studies, with 1404 cases of hypertension out of 1733 COVID-19 patients, resulting in a prevalence of $83 \%$ (95\% CI, 78-88\%). Overall, the prevalence of COVID-19 was $23 \%$ (95\% CI, 17-28\%) in kidney transplant recipients with underlying heart disease. Based on 14 studies, the estimated pooled prevalence of obesity was $36 \%(95 \% \mathrm{CI}, 26-46 \%)$; positive smoking status $15 \%$ (95\% CI, $10-20 \%)$; chronic lung disease $15 \%$, (95\% CI, 5-24\%); and malignancy $8 \%$ (95\% CI, $6-11 \%)$.

\subsection{Clinical Characteristics and Laboratory Results}

The pooled estimate, with $95 \% \mathrm{CI}$ was determined via random-effect analysis of the reported proportions. The most predominant clinical feature reported during hospitalization was fever, which was identified in $77 \%$ (95\% CI, 72-81\%) of patients, followed by cough $65 \%$ (95\% CI, 61-69\%), dyspnea $48 \%$ (95\% CI, 42-53\%), and gastrointestinal symptoms $29 \%$ (95\% CI, 25-34\%). Several articles have noted that higher levels of inflammatory markers equate with increased disease severity and a poor prognosis in COVID-19 infected patients [67]. The pooled proportion of patients with lymphocytopenia $\left(<1000 \mathrm{cells} / \mathrm{mm}^{3}\right)$ and high CRP ( $>5 \mathrm{mg} / \mathrm{dL}$ ) was 79\% (95\% CI, 70-89\%) and 60\% (95\% CI, 35-84\%), respectively. The pooled prevalence of radiological evidence of pneumonia was documented in $81 \%(95 \% \mathrm{CI}, 74-88 \%)$ of patients (Table A4).

\subsection{Immunosuppression Modulation and COVID-19 Drug Treatment}

The pooled prevalence of patients who received hydroxychloroquine and tocilizumab was $73 \%$ (95\% CI, 66-80\%) and 21\% (95\% CI, 16-26\%), respectively (Table A4). Data from relevant studies reporting modifications to immunosuppression were extracted and analyzed. Mycophenolate mofetil/mycophenolic acid (MMF/MPA) was the most commonly withheld or reduced immunosuppressive medication in $91 \%$ (95\% CI, 88-94\%) of patients 
followed by CNI $43 \%$ (95\% CI 30-56\%). Additionally, pulse steroids were administered in $40 \%(95 \%$ CI, $30-50 \%)$ of patients.

\subsection{Disease Severity and Mortality}

Based upon data available in the analyzed studies, the pooled prevalence of COVID19 associated hospital mortality was $21 \%$ (95\% CI, 19-24\%) for inpatients (718 of 3137) with a significantly higher mortality of 53\% (95\% CI, 44-63\%) among patients admitted to the intensive care unit (ICU) (184 of 355) $(p<0.0001)$ (Figure 3a,b). An ICU admission was required in $26 \%$ (95\% CI, 22-30\%) of kidney transplant recipients hospitalized with COVID-19, albeit data might not be representative of the actual requirement of ICU-level care due to limited availability in the midst of the global pandemic (Figure 3c). The pooled prevalence of ARDS and AKI in COVID-19 infection was 58\% (95\% CI, 48-69\%) and 48\% ( $95 \%$ CI, $43-53 \%$ ), respectively (Figure 3d). Similarly, the pooled prevalence of mechanical ventilation was $24 \%$ (95\% CI, 20-28\%) (Figure 3e) and of dialysis was 22\% (95\% CI, 16-29\%). Furthermore, the mortality among those who required mechanical ventilation was $68 \%$ (95\% CI, 58-79\%) for inpatients (206/285) (Figure 3f).

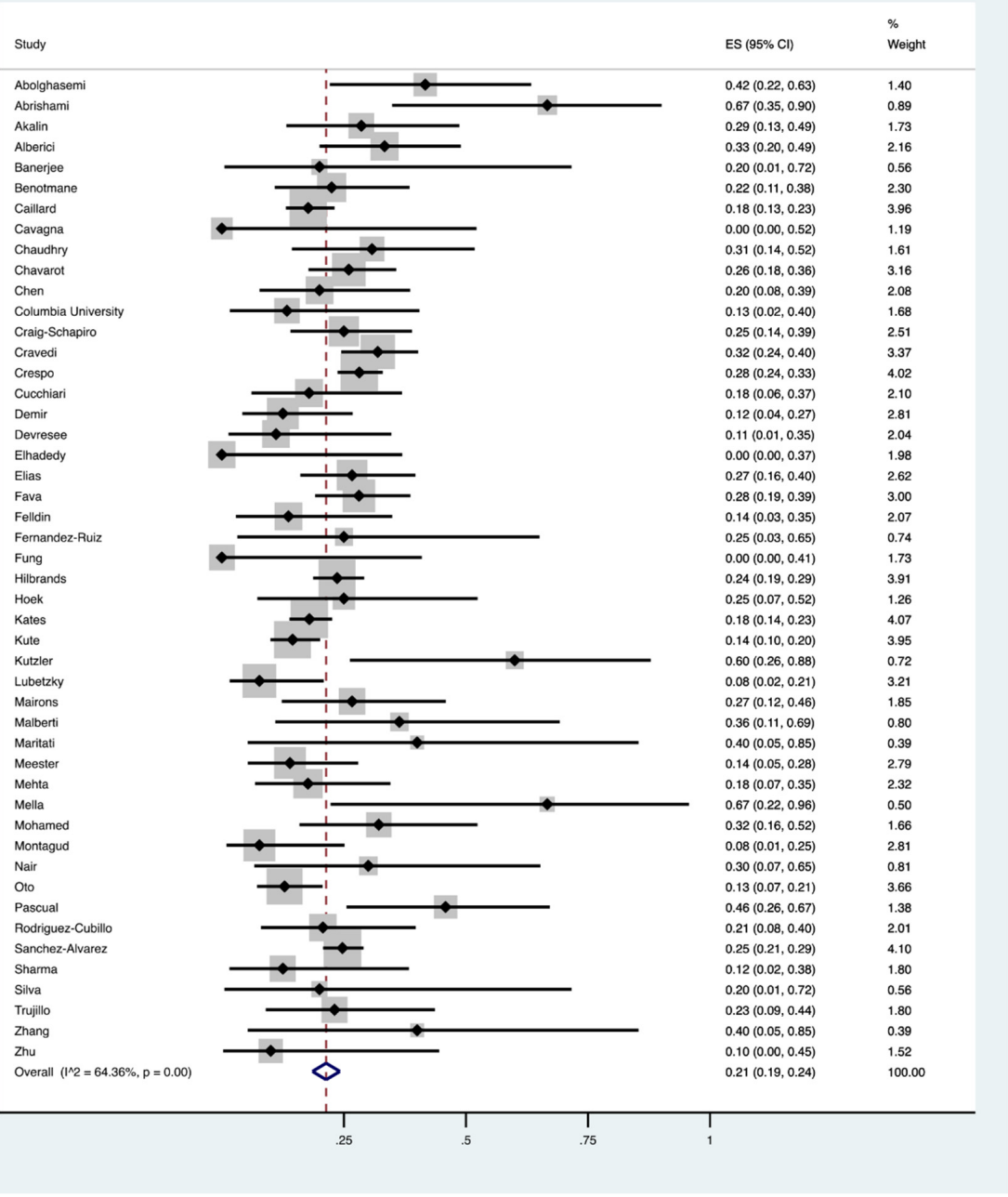

(a)

Figure 3. Cont. 


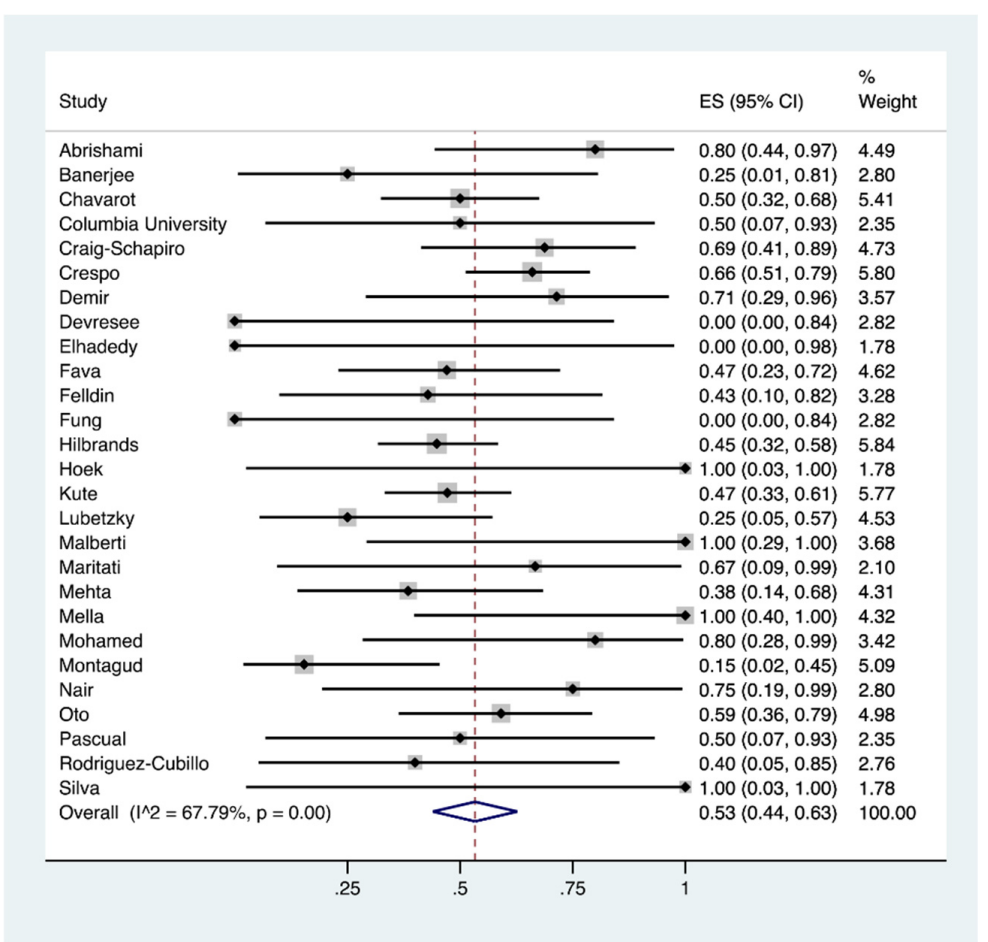

(b)

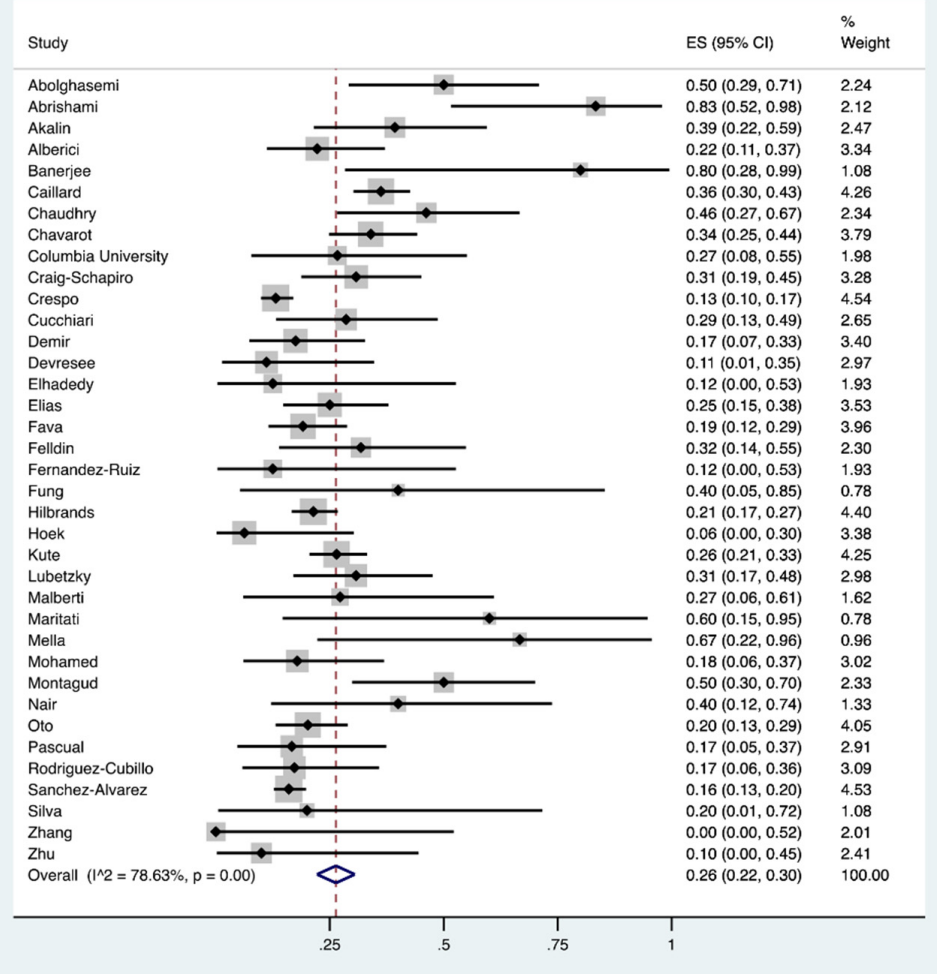

(c)

Figure 3. Cont. 


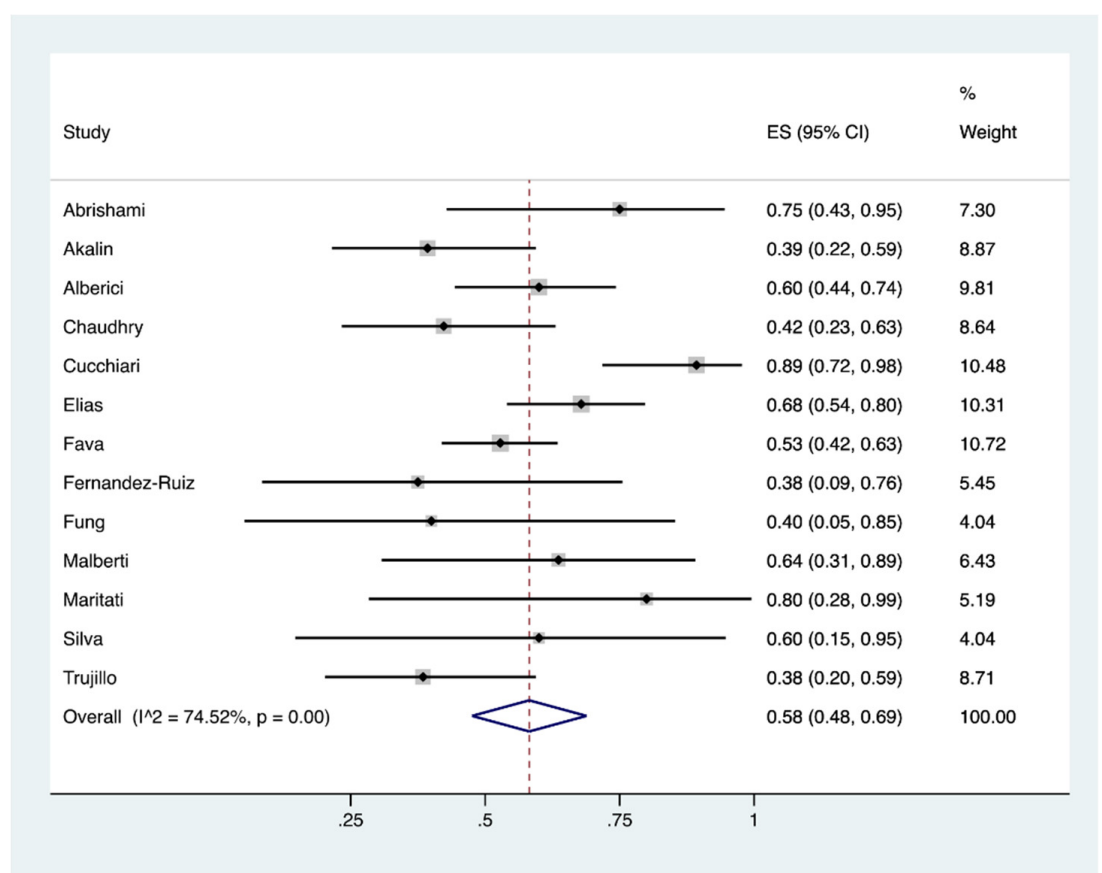

(d)

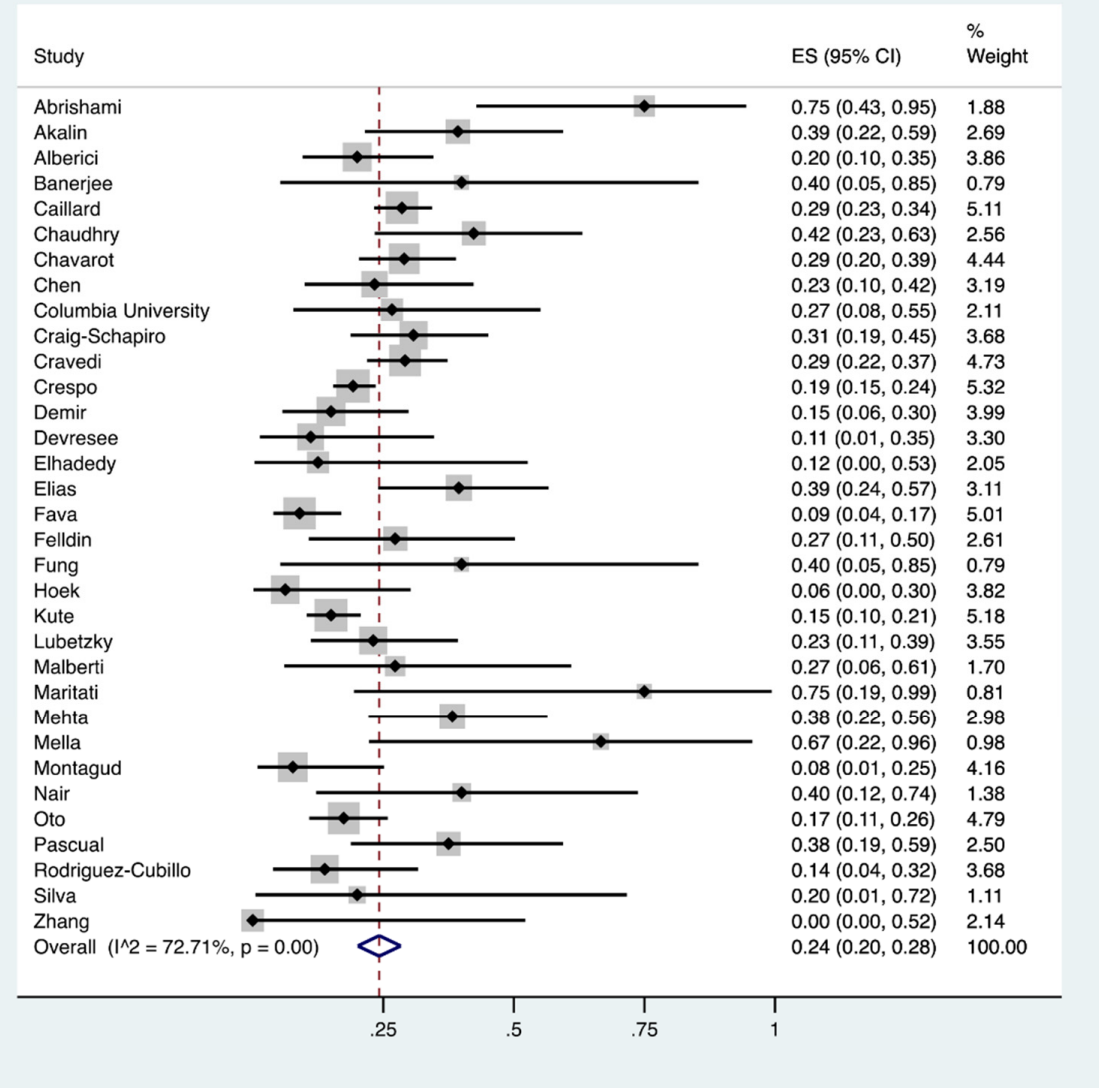

(e)

Figure 3. Cont. 


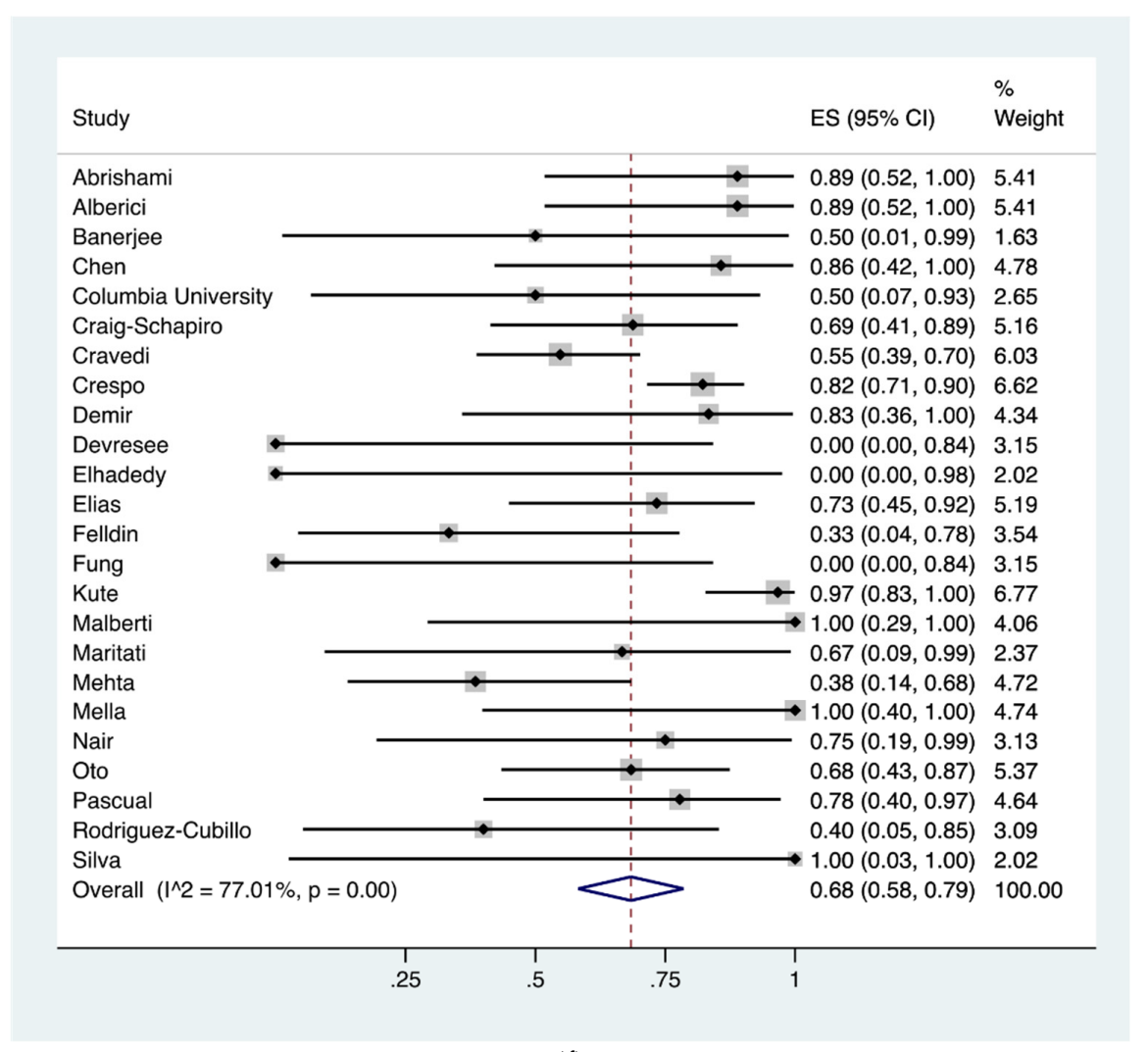

(f)

Figure 3. (a) Pooled prevalence of in-hospital mortality in kidney transplant recipients diagnosed with corona virus disease 2019 (COVID-19). In-hospital mortality was outlined in 718/3137 patients (48 studies). The vertical red dashed line represents the overall mean effect size of the studies (0.22) and prevalence of $22 \%$. The edges of the blue diamond represent 95\% confidence intervals (0.19-0.24); (b) Pooled prevalence of intensive care unit (ICU) mortality in kidney transplant recipients diagnosed with COVID-19. ICU mortality was outlined in 184/355 patients (24 studies). The vertical red dashed line represents the overall mean effect size of the studies (0.53) and prevalence of $53 \%$. The edges of the blue diamond represent 95\% confidence intervals (0.44-0.63); (c) Pooled prevalence of intensive care unit (ICU) admission in kidney transplant recipients diagnosed with COVID-19. ICU admission data were required in 570/2439 patients (37 studies). The vertical red dashed line represents the overall mean effect size of the studies (0.26) and a prevalence of $26 \%$. The edges of the blue diamond represent 95\% confidence intervals (0.22-0.30); (d) Pooled prevalence of acute respiratory distress syndrome (ARDS) in kidney transplant recipients diagnosed with COVID-19. ARDS was present in 197/344 patients as reported in 13 studies. The vertical red dashed line represents the overall mean effect size of the studies (0.58) and a prevalence of $58 \%$. The edges of the blue diamond represent $95 \%$ confidence intervals $(0.48-0.69)$; (e) Pooled prevalence of mechanical ventilation requirement in kidney transplant recipients diagnosed with COVID-19. Mechanical ventilation was needed in $433 / 1848$ patients (33 studies). The vertical red dashed line represents the overall mean effect size of the studies (0.24) and prevalence of $25 \%$. The edges of the blue diamond represent $95 \%$ confidence intervals (0.20-0.28); (f) Pooled prevalence of mortality while on invasive ventilation in kidney transplant recipients diagnosed with COVID-19. Mortality in patients requiring invasive ventilation was 206/285 as reported (24 studies). The vertical red dashed line represents the overall mean effect size of the studies (0.68) and prevalence of $68 \%$. The edges of the blue diamond represent $95 \%$ confidence intervals (0.58-0.79).

Moreover, a group-wise comparison was made to determine the association of mortality and ARDS in the index population. The analysis revealed significantly higher risk of mortality in COVID-19 patients with ARDS in contrast to patients without ARDS (OR = 19.59; 95\% CI, 6.64-57.78) (Figure 4a). Similarly, our analysis showed a significantly higher mortality risk in kidney transplant recipients on mechanical ventilation following COVID-19 (OR = 3.80; 95\% CI, 2.35-6.14) (Figure 4b). Furthermore, our analysis demonstrated a significantly higher mortality risk in patients' receiving ICU care (OR $=13.39 ; 95 \% \mathrm{CI}, 7.27-24.68)$ (Figure 4c). 


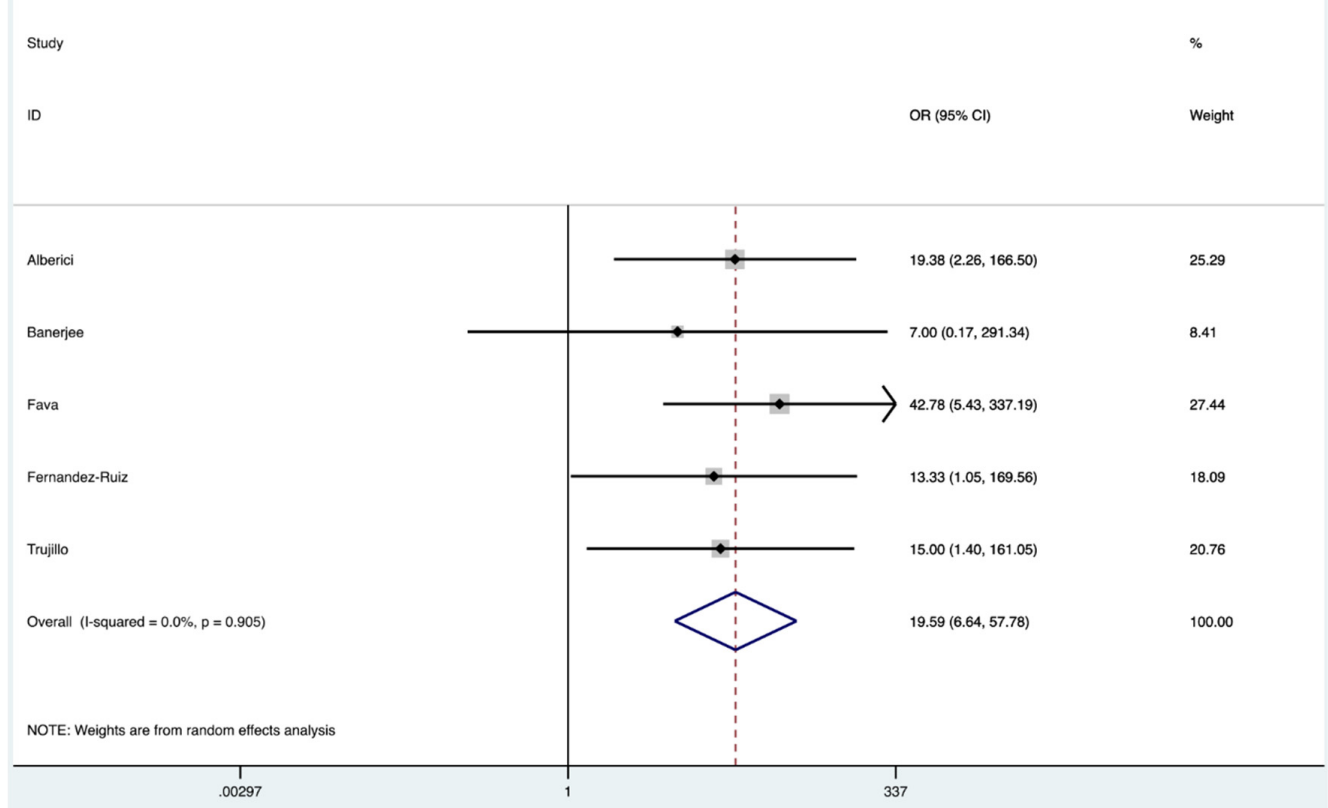

(a)

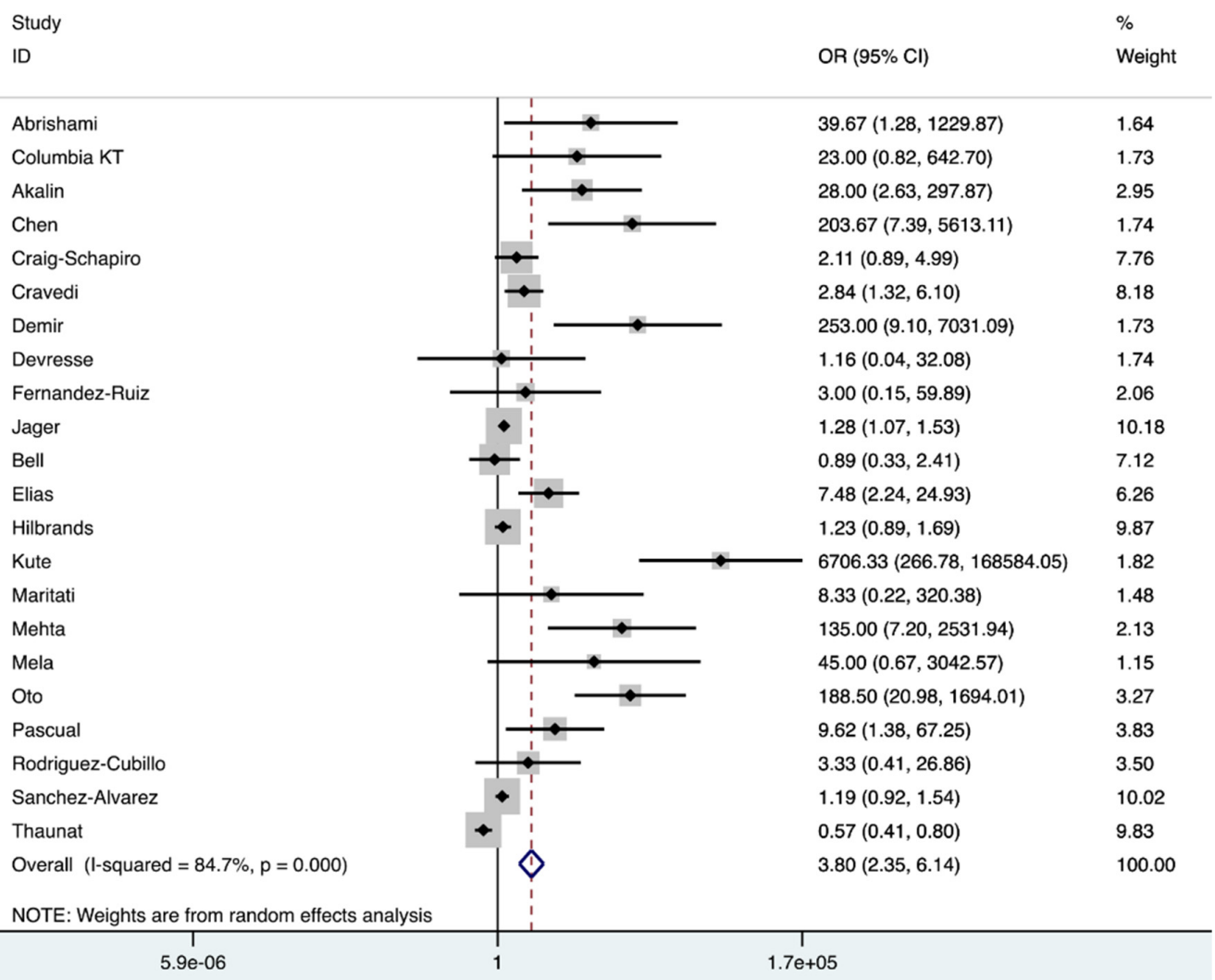

(b)

Figure 4. Cont. 


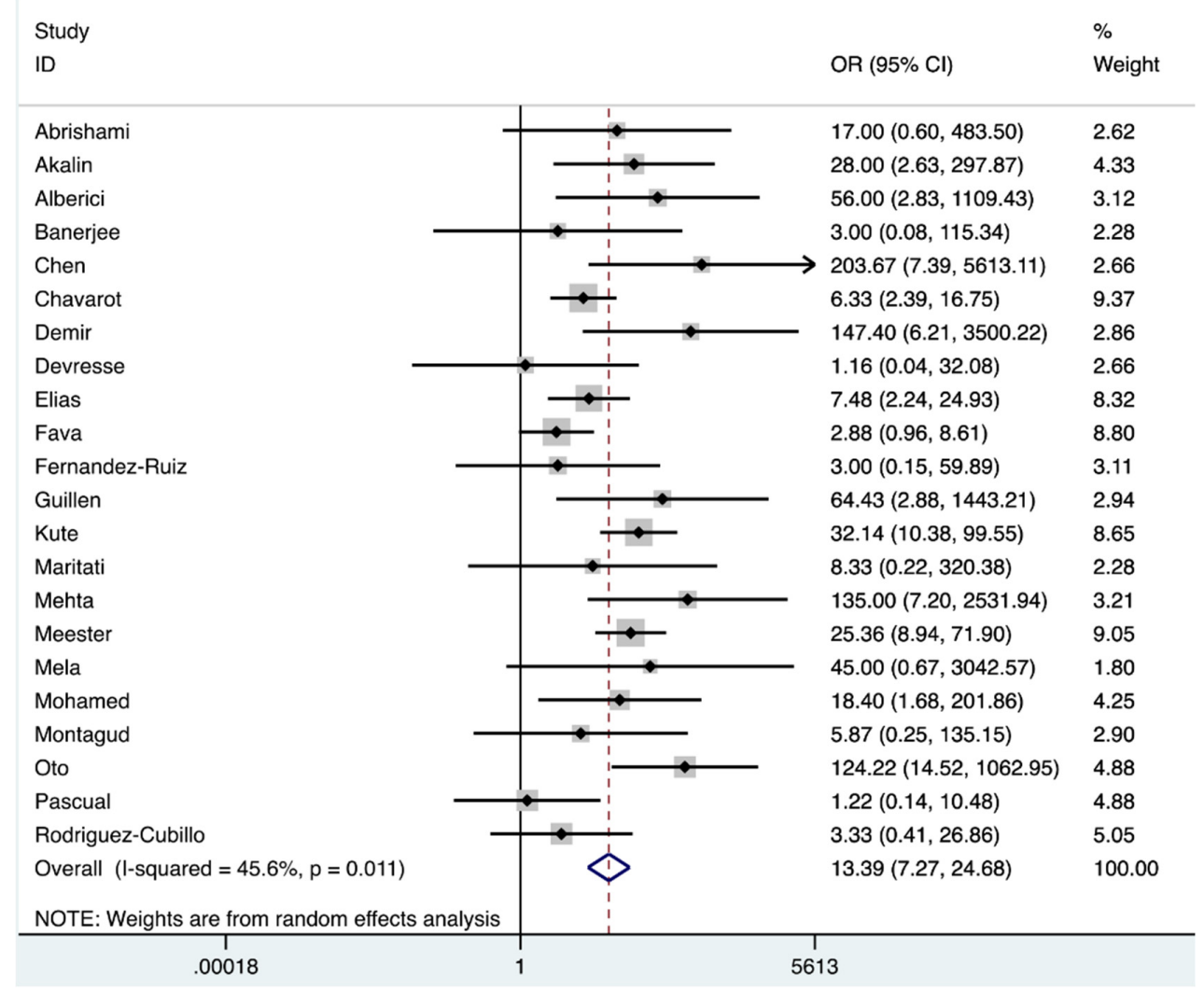

(c)

Figure 4. (a) Forest plot depicting COVID-19 associated mortality risk in kidney transplant recipients with ARDS in contrast to no ARDS. The diamond shows increased risk in patients with ARDS, OR = 19.59 (red dashed line). The edges of the blue diamond represent 95\% confidence intervals (6.64-57.78). ARDS: Acute respiratory distress syndrome; (b) Forest plot depicting COVID-19 associated mortality risk in kidney transplant recipients on invasive ventilation in contrast to not on invasive ventilation. The diamond shows increased risk in patients receiving invasive ventilation, OR 3.80 represented by red dashed line with the edges of the blue diamond representing 95\% confidence intervals (2.35-6.14); (c) Forest plot depicting COVID-19 associated mortality risk in kidney transplant recipients receiving critical care in contrast to hospital admission without critical care. The diamond shows increased risk in patients receiving critical care group, OR 13.39 represented by red dashed line with the edges of the blue diamond representing 95\% confidence intervals (7.27-24.68). OR: Odds ratio.

Additionally, a group-wise comparison of mortality was conducted for the following attributes: age ( $\geq 60$ years vs. $<60$ years), time since kidney transplantation $(<2$ years vs. $\geq 2$ years), and reported gender (male vs. female). The odds ratio of COVID-19 related death was significantly higher in the age group $\geq 60$ years ( $\mathrm{OR}=3.90 ; 95 \% \mathrm{CI}, 2.56-5.94)$; however, neither such association existed for time since transplant (i.e., $\geq 2$ years) $(\mathrm{OR}=1.37 ; 95 \% \mathrm{CI}$, $0.72-2.58)$ nor for gender (OR $=0.71 ; 95 \% \mathrm{CI}, 0.50-1.01)$ (Figure $5 \mathrm{a}-\mathrm{c}$ ). 


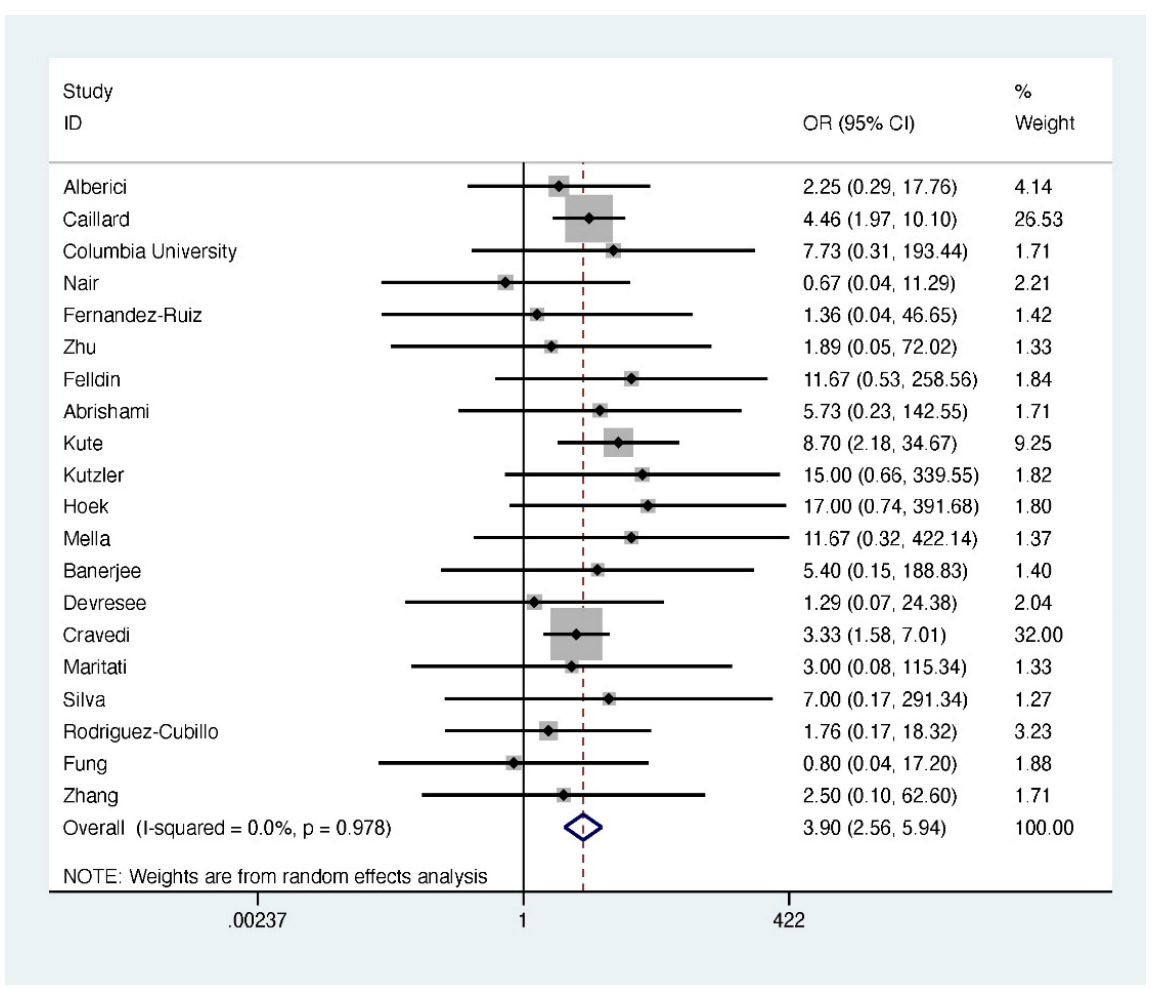

(a)

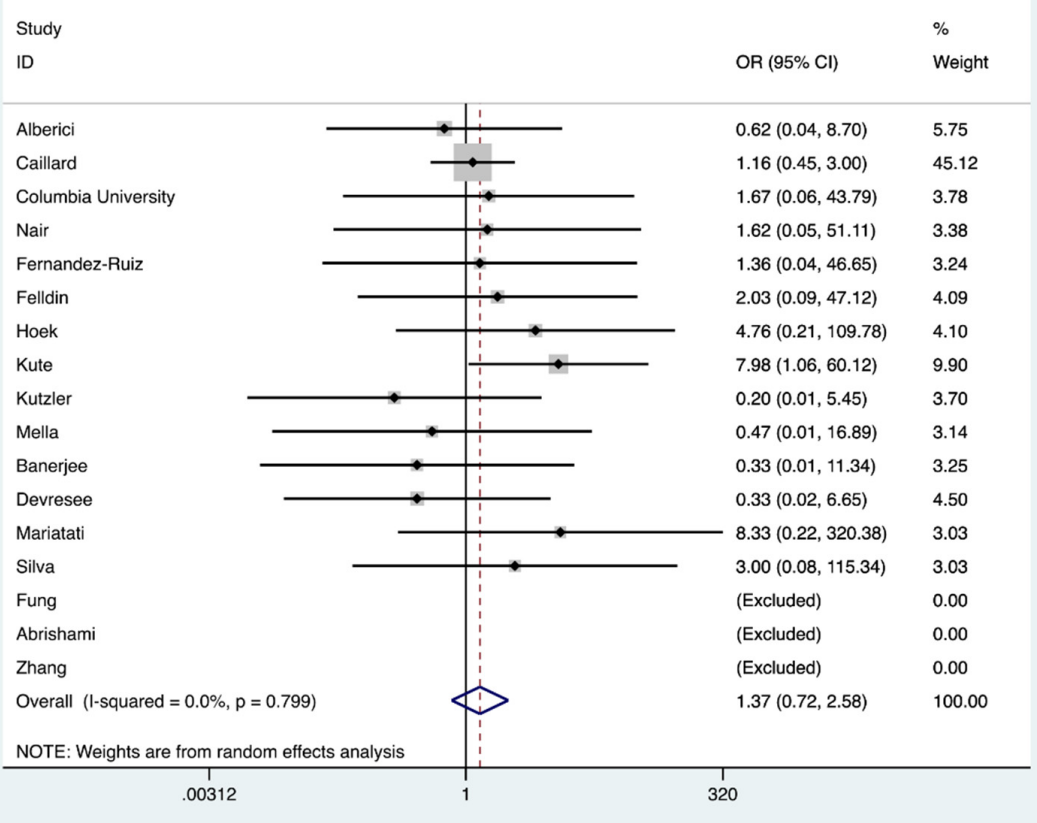

(b)

Figure 5. Cont. 


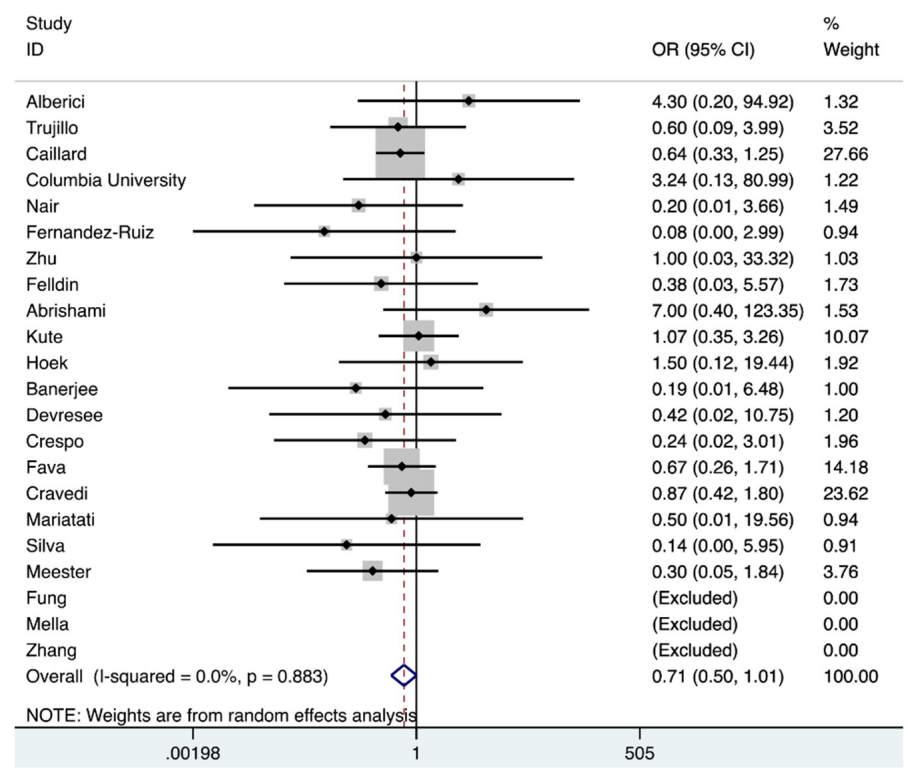

(c)

Figure 5. (a) Forest plot depicting COVID-19 associated mortality risk in kidney transplant recipients. The diamond shows higher risk in patient group $\geq 60$ years of age, $\mathrm{OR}=3.90$ (red dashed line). The edges of the blue diamond represent $95 \%$ confidence intervals (2.56-5.94); (b) Forest plot depicting COVID-19 associated mortality risk in kidney transplant recipients in the late post-transplant period group ( $>2$ years) in contrast to the early post-transplant period ( $\leq 2$ years). The diamond shows no increased risk between the groups; OR 1.37 represented by red dashed line with the edges of the blue diamond representing 95\% confidence intervals (0.72-2.58); (c) Forest plot depicting sex related mortality risk in COVID-19 suffering kidney transplant recipients. The diamond shows no increased risk between the groups; OR 0.71 represented by vertical red dashed line with the edges of the blue diamond representing 95\% confidence intervals (0.50-1.01). OR: Odds ratio.

Based upon the availability of data in the included studies, comparative mortality risk following COVID-19 was assessed in CKD patients on the waitlist or on dialysis in comparison to kidney transplant recipients and analysis demonstrated no significant increased risk OR $=1.24$ (95\% CI, 0.92-1.66) (Figure 6).

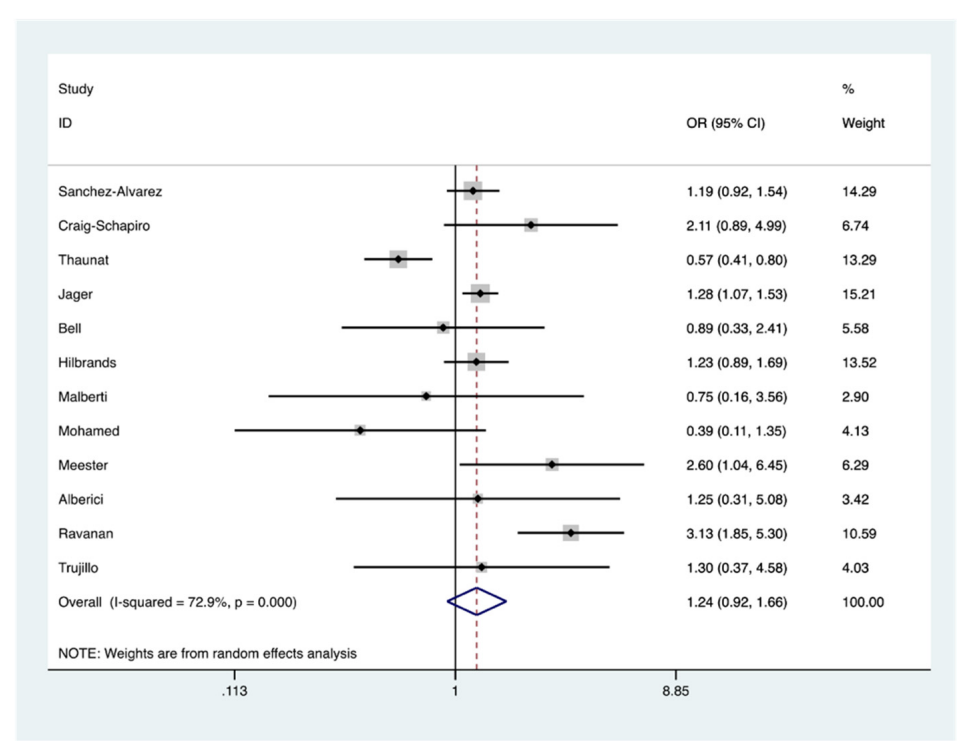

Figure 6. Forest plot depicting COVID-19 associated mortality risk in chronic kidney disease (CKD) patients on waitlist/dialysis in contrast to kidney transplant recipients. The diamond shows no increased risk of death; OR 1.24 represented by red dashed line with the edges of the blue diamond representing $95 \%$ confidence intervals (0.92-1.66). OR: Odds ratio. 


\section{Discussion}

Kidney transplantation provides benefits of improved quality of life and reduced mortality compared to other renal replacement therapies [68-70]. Since the beginning of the pandemic, there has been sense of disquietude that kidney transplant recipients might predispose to abysmal outcomes after SARS-CoV-2 infection and as the pandemic evolved, most policy-makers have advised minimizing social contacts. A detailed understanding of COVID-19 susceptibility, disease process and outcomes has been essential to create a customized health advice to this subset of population.

First, transplant-associated immunosuppression renders patients generally at an increased risk of infection. Early reports suggest that risk is also true for SARS-CoV2. Second, SARS-CoV-2 has been shown to have an affinity for angiotensin converting enzyme 2 (ACE2) receptors, which are predominantly found in the lungs and kidneys and this may be partially responsible for organ injury secondary to the infection. Additionally, uncertainty and fear of the sequalae of COVID-19 infection have resulted in a significant decline in renal transplantation, particularly during the initial stage of the pandemic $[4,5]$.

The exact mechanism by which the viral infection causes kidney injury has not been elucidated. One leading proposed mechanism is that the virus causes direct damage to the renal tubular epithelium and podocytes via an angiotensin converting enzyme 2 mediated process, which leads to a cascade of mitochondrial dysfunction, acute tubular necrosis, formation of protein resorption vacuoles, protein leakage in Bowman's capsule, and collapsing glomerulopathy [71,72]. Viremia may also directly lead to the damage of endothelial cells in the kidney [73]. The severe inflammatory response in the setting of dysregulated immune activation and a cytokine syndrome following SARS-CoV-2 infection might prompt acute respiratory syndrome, septic shock, and multi-organ failure including acute renal failure [74]. Other contributing factors include macrophage activation syndrome, endothelilitis, and hypercoagulability, leading to microthrombus formation, micro-embolism, and rhabdomyolysis [75].

Some insights into the mechanism of injury may be elucidated by analysis of the clinical evolution of infected patients. According to the WHO-China joint commission report on COVID-19 in the general population, fever $(88 \%)$ and cough $(68 \%)$ are reported to be the most common initial symptoms [76]. Interestingly, despite a decreased immune response secondary to chronic immunosuppression, we observed a similar rate cough $(65 \%)$ among kidney transplant recipients with COVID-19. However, our analysis revealed a lower prevalence of fever ( $77 \%$ vs. $88 \%)$, but higher prevalence of dyspnea ( $48 \%$ vs. $18.6 \%)$ and gastrointestinal symptoms (29\% vs. $3.7 \%)$ when compared to the general population studied in the WHO report [76]. A plausible explanation of the lower prevalence of fever could be because of the immunosuppressive state, whilst higher prevalence of gastrointestinal symptoms in the index cohort might be due to an exacerbation of the known toxic effects of antimetabolites on the gastrointestinal tract. Furthermore, the increased frequency of dyspnea in kidney transplant (KT) recipients could be compounded by the 10 -fold higher prevalence of chronic lung disease (15\%) among KT recipients compared to $1.5 \%$ in the general population with COVID-19 [77].

The mortality rate of SARS-CoV-2 in hospitalized patients as reported in recent metaanalysis of 14 studies was $4.8 \%$ [13]. Importantly, several risk factors for increased mortality have been identified including male sex (ORs $=1.50)$, advanced age (ORs $=4.59)$, hypertension $(\mathrm{ORs}=2.70)$, cardiac disease $(\mathrm{CVDs})(\mathrm{ORs}=3.72)$, T2DM $(\mathrm{ORs}=2.41)$, lung disorders $(\mathrm{ORs}=3.53)$, or malignancy $(\mathrm{ORs}=3.04)$ [13]. The observed mortality rate among kidney transplant recipients hospitalized due to COVID-19 in our meta-analysis was $21 \%$; although there have been wide inconsistencies throughout the literature regarding mortality data as several studies have been published upon in-complete follow-up, hence mortality figures could be higher and future studies with complete follow-up are required to completely elucidate the disease process.

Multitudes of studies have outlined the association of higher case-fatality with COVID19 to increased prevalence of comorbidities [78-80]. Yang et al. performed a meta-analysis 
of seven studies including 1576 patients with COVID-19 and determined that the most common comorbidities were hypertension $(21.1 \%)$, diabetes $(9.7 \%)$, cardiovascular disease $(8.4 \%)$, and chronic lung disease (1.5\%) [77]. Our meta-analysis demonstrated over $\sim 3-4$ fold higher prevalence of these comorbidities among kidney transplant recipients and COVID-19 with numbers being $83 \%$ for hypertension, $34 \%$ for T2DM, $23 \%$ for cardiac disease, and $15 \%$ or $~ 10$ fold higher for chronic lung diseases. When considering the attributed risk of age, our analysis revealed a statistically significant heightened risk in the elderly population (OR 3.90) with no increased risk when comparing sex and time since transplant after which infection was acquired. Thus, this group remains a vulnerable population due to increasing age and associated comorbidities.

Some reports have demonstrated that the natural course of COVID-19 may lead to multiorgan failure in certain subsets of the population. with regard to renal injury, a recent meta-analysis reported that $17 \%(0.5-80.3 \%)$ of patients had acute kidney insufficiency (AKI) with 5\% (0.8-14.7\%) requiring hemodialysis (HD) [81]. However, the observed prevalence of $\mathrm{AKI}$ and $\mathrm{HD}$ requirement in our study was much higher (i.e., $48 \%$ and $22 \%$, respectively). These findings are consistent with factors specific for kidney transplant recipients including lower functional reserve of kidney allograft, and the toxic effect of tacrolimus in combination with increased susceptibility to prerenal causes of renal dysfunction (dehydration, hypotension, and metabolic disarray), which are absent in the general population.

For COVID-19 patients, clinical outcomes in kidney transplant recipients are worse than in non-transplant patients overall, however, they seem to be comparable to patients with end stage renal disease (ESRD). Two studies from the same medical center in New York City, NY reported a comparable case-fatality rate of 25\% for both ESRD or kidney transplant recipient COVID-19 positive patients [82,83].

The high comorbidity rate in ESRD patients and kidney transplant recipients are wellrecognized attributes, resulting in a similarly elevated risk of acute organ injury during SARS-CoV-2 infection, and also a consequently higher mortality risk than the general population [78,84-86]. A retrospective study involving 3988 critically ill patients from Lombardy, Italy reported increased mortality in CKD patients (i.e., 41 (78.8\%) out of 52 patients and univariate analysis revealed HR 2.78 (95\% CI; 2.19-3.53) [87]), although our meta-analysis demonstrated no significant increased mortality risk $(\mathrm{OR}=1.24 ; 95 \% \mathrm{CI}$, 0.92-1.66) when both were compared. Fortunately, this observation demands further studies as recent study from NHS England "OpenSAFELY" has outlined causal factors for 10,926 COVID-19 related deaths and reported CKD as an important attribute for mortality. Furthermore, when the data from the CKD subgroups were compared, ESRD and CKD at stage 5 (HR 2.52) were found to be associated with higher COVID-19 related mortality than other isolated chronic ailments such as hypertension, obesity, cardiac disease, or chronic respiratory disease [88]. However, the risk of contracting SARS-CoV-2 in patients attending dialysis sessions three times a week (and cumulative risk from inherent comorbidities of not being transplanted) needs to be weighed against risk of immunosuppression, especially in acute transplantation. For the latter, the risk of surgery, cardiovascular mortality, infection, and increased immunosuppression burden will be elevated for some months before reaching equipoise.

A summary of studies outlined outcomes in critically ill COVID-19 patients and reported a higher mortality for the non-transplanted patients. The mortality rate among over 10,000 critically ill non-transplant patients was reported to be as high as $41 \%$ in a meta-analysis of 24 studies by Armstrong et al. [89]. However, critical care unit mortality for patients with similar characteristics in a transplant cohort reached as high as $53 \%$ with a stepwise increase in frequency of ICU admission (26\%), invasive ventilation (24\%), and mortality of $68 \%$ whilst on a mechanical ventilator. The estimated prevalence of ARDS in our meta-analysis was $58 \%$ (95\% CI, $48-69 \%)$ and $24 \%$ of KT patients required invasive ventilation, which was comparable to the non-transplant patient cohorts in which $41.8 \%$ of 201 hospitalized COVID-19 patients developed ARDS [85] with the number rising to $68 \%$ 
in critical care settings [79]. Thus, the trajectory of mortality in kidney transplant recipients showed a significant upward trend, with those requiring critical care support and was further high in patients with respiratory failure, although explicit details underpinning this observation remain elusive. However, it is plausible that the hypercoagulable state of CKD along with pulmonary thromboembolic disease of critical COVID-19 are mounting the risk of thrombotic complication and increased mortality. Hence, enhanced thromboembolic prophylaxis should be considered in hospitalized cohorts of COVID-19 patients, although at present, wide variations in clinical guidelines exist and unified disease management protocol with risk stratification are yet to be established.

Inefficiency of the health system in response to the initial face of the pandemic, in addition to major concerns about the detrimental effect of the immunosuppression on a clinical course of COVID-19, has led to halting kidney transplantation procedures in most programs globally. However, delaying kidney transplantation also has negative consequences. A recent study reported a 2.2-fold rise in mortality rate in CKD patients on the wait-list for transplantation during the ongoing pandemic [10]. A study of the Spanish Society of Nephrology Registry reported higher rates of SARS-CoV-2 infection and a $23 \%$ mortality rate among ESRD patients on hemodialysis [90]. Moreover, no direct evidence is available to suggest that immunosuppression has a negative effect on the clinical course of COVID-19 in kidney transplant recipients and hence, despite the on-going pandemic, clinical transplantation services around the world have largely resumed following the initial interruption. Of note, most of the reports included in our metanalysis presented outcomes during the first phase of the pandemic and the high mortality rates reported may also be related to the transient but drastic limitations of vital resources like critical care capacity [89]. At the same time, COVID-19 vaccines were not available and the Delta variant not described. Therefore, our study provides insight on the impact of COVID-19 on kidney transplant population in such conditions. Nevertheless, it provides valuable information and may serve as a reference for future studies assessing the impact of patient vaccination as well as the Delta variant of COVID-19 in the kidney transplant population in the future.

At the beginning of the pandemic, SARS-CoV-2 infection was thought to pose a significant challenge for kidney transplant recipients due to the immunosuppressive state; however, the current understanding suggests that the immunosuppression by itself does not seem to confer an increased risk of poor outcome in SARS-CoV-2 infection, which is consistent with other observations. First, typical immunosuppression for kidney transplant recipients primarily limits the adaptive immune response rather than the innate response, with the latter seeming primary in determining COVID-19 outcome. The only form of immunosuppression strongly linked with poor outcomes identified to date is of specific deficits in the type I interferon innate response. Second, reactive corticosteroid immunosuppression with high-dose dexamethasone reduces mortality in severe COVID-19. Third, as noted by others, immunosuppression, did not emerge as a risk factor for poor outcome in SARS-CoV-1 or Middle Eastern Respiratory Syndrome infections, whereas comorbidities were analogous to those conferring risk in SARS-CoV-2 infection [91-93]. Interestingly, the fact that kidney transplant recipients have similar outcomes to the matched general population is in stark contrast to the very high rates of mortality reported in those with ESRD. This finding might suggest that, in the context of COVID-19, risk of ESRD and associated mortality has a more deleterious effect than pharmacological immunosuppression. It therefore remains the case that major international guidelines currently recommend against the routine cessation or reduction in immunosuppressive therapy in KT recipients before any SARS-CoV-2 infection and that modification following SARS-CoV-2 be considered under special circumstances such as in superadded bacterial infection or worsening respiratory failure. Consequently, subsequent CD8+ T cell and B cell recovery has been observed with progressive resolution of the disease $[94,95]$. Hence, some degree of reinstatement of a functioning immune system may be desired in KT recipients in order to limit the severity of host response to SARS-CoV-2 infection. Neither the lymphopenia observed in many 
patients with COVID-19 nor the established practice of antimetabolite dose reduction or even cessation during the treatment of other viral infections in transplant patients (cytomegalovirus, BK virus infections) have led to increased rates of rejection [96-99]. Recent reports have recognized the utility of steroids in critically ill COVID-19 patients, which may limit the severity of ARDS and the associated cytokine storm [100-102]. Consequently, the RECOVERY trial outlined the benefit of high dose dexamethasone in critically ill COVID-19 patients on mechanical ventilation [103]. Thus, there is some basis for the administration of pulsed steroids doses as was given to $41 \%$ of the patients in our meta-analysis. There are few limitations of our meta-analysis. The relatively large number of small population studies has made our composite data heterogeneous despite using a random effect model for analysis. Additionally, differences in reported clinical trajectories, management approach, and outcomes is likely to be significantly dependent on the available resources and other variable factors such as admission criteria across the different health care facilities reporting the cases.

The strengths of this meta-analysis are the extensive and exhaustive nature of the search, the independent process of study selection and data abstraction, and the random effects model used for the analysis of observational studies. We hope that this meta-analysis will contribute to a more detailed understanding of the SARS-CoV-2 disease process in the KT patient and guide future clinical decision making for this patient population.

In summary, the clinical presentation of COVID-19 in kidney transplant recipients seems to differ from the general population with lower prevalence of fever and a higher prevalence of dyspnea and gastrointestinal symptoms. The clinical course of COVID-19 in kidney transplant recipients revealed higher mortality in different settings of health care facilities including hospital, ICU, and those requiring invasive ventilation. within the cohorts' studies of kidney transplant recipients, age and presence of multitudes of comorbidities were strongly associated with complicated disease course (i.e., increased prevalence of ARDS, AKI, and HD requirement and mortality). Whilst data regarding impact of SARS-CoV-2 in this cohort are still accumulating; however, to date, it seems clear that weight of comorbidity are the most important attributes in determining the outcome than transplant surgery alone. As being in an immunosuppressed state constitutes less risk from SARS-CoV-2 than ESRD patients on hemodialysis, by that mean, we advocate for the continuation of kidney transplantation programs.

Author Contributions: Concept and study design-P.W. and K.J.; Methodology and data acquisitionK.J., I.R., and K.J.; Data analysis and interpretation-P.W., K.J., I.R., M.P., Y.A.-S., J.S.P., P.J.B., and A.P.-G.; Drafting of the article-P.W., Y.B., D.d.S., J.L., R.B., J.F., F.J.M.F.D., K.J., I.R., Y.A.-S., M.P., J.S.P., and P.J.B.; Critically revised the article-P.W., K.J., I.R., Y.A.-S., M.P., A.P.-G., F.J.M.F.D., Y.B., D.d.S., J.L., R.B., J.F., J.S.P., and P.J.B. All authors have read and agreed to the published version of the manuscript.

Funding: This research received no external funding.

Institutional Review Board Statement: Not applicable.

Informed Consent Statement: Not applicable.

Data Availability Statement: No new data were created in this study.

Conflicts of Interest: The authors declare no conflict of interest. 


\section{Appendix A}

Table A1. Baseline demographic and clinical data of post-kidney transplant COVID-19 patients in a sequence of publication time.

\begin{tabular}{|c|c|c|c|c|c|c|c|c|c|c|}
\hline Study & Country & $\begin{array}{l}\text { Number of } \\
\text { Patients in the } \\
\text { Study }\end{array}$ & $\operatorname{Sex}(M / F)$ & Age (Years) & Ethnicity & Donor Type & Comorbidities & $\begin{array}{l}\text { Medications (Immunosuppression } \\
\text { + Others) }\end{array}$ & $\begin{array}{l}\text { Time Since } \\
\text { Renal-Transplant, } \\
\text { Median (Range) or } \\
\text { Mean } \pm \text { SD }\end{array}$ & $\begin{array}{l}\text { Baseline Serum } \mathrm{Cr}_{\mathrm{r}} / \mathrm{d} \\
\text { Median (Range) mg/dL }\end{array}$ \\
\hline Akalin et al. [19] & United States & 36 & $26(72 \%) / 10(28 \%)$ & $57.25 \pm 12.9$ & $\begin{array}{l}\text { African American: } 14 \\
\text { (39\%) } \\
\text { Hispanics: } 15(42 \%) \\
\text { Others: } 7(19 \%)\end{array}$ & $\begin{array}{l}\text { Deceased: } 27(75 \%) \\
\text { Living: } 9(25 \%)\end{array}$ & $\begin{array}{l}\text { HTN: } 34(94 \%) \\
\text { DM: } 25(69 \%) \\
\text { CAD: } 6(17 \%) \\
\text { Lung disease: } 4(11 \%) \\
\text { Cancer: } 2(6 \%) \\
\text { Smoking: } 6(17 \%)\end{array}$ & $\begin{array}{l}\text { Tacrolimus: } 35(97 \%) \\
\text { Prednisone: } 34(94 \%) \\
\text { MMFF MPA: } 31(86 \%) \\
\text { ARB: } 8(22 \%)\end{array}$ & $\mathrm{NA}$ & $2.4 \pm 1.63$ \\
\hline Bossini et al. [20] & Italy & 53 & $42(79 \%) / 11(21 \%)$ & $59.25 \pm 4.9$ & $\mathrm{NA}$ & $\begin{array}{l}\text { Deceased: } 48(91 \%) \\
\text { Living: } 5(9 \%)\end{array}$ & $\begin{array}{l}\text { ITN: } 42(79 \%) \\
\text { DM: } 11(21 \%) \\
\text { CAD : 10 (19\%) } \\
\text { Previous DVT: } 4(8 \%)\end{array}$ & $\begin{array}{l}\text { CNIs: } 48(90 \%) \\
\text { Prednisone: } 30(57 \%) \\
\text { MMF/MPA: } 32(60 \%) \\
\text { mTORi: } 6(11 \%)\end{array}$ & $9.6 \pm 3.5$ years & $1.89 \pm 0.33$ \\
\hline Trujillo et al. [21] & Spain & 26 & $12(46 \%) / 14(54 \%)$ & $61 \pm 14$ & $\begin{array}{l}\text { Caucasian: } 22(85 \%) \\
\text { Hispanics: } 4(15 \%)\end{array}$ & $\mathrm{NA}$ & 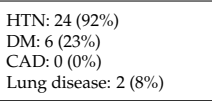 & $\begin{array}{l}\text { CNIs: } 24 \text { (92\%) } \\
\text { Prednisone: } 22(84 \%) \\
\text { MMF/MPA: } 14(54 \%) \\
\text { mTORi: } 7(27 \%)\end{array}$ & $7(4-15)$ years & $1.9 \pm 0.33$ \\
\hline $\begin{array}{l}\text { Columbia University } \\
\text { KT program [22] }\end{array}$ & United States & 15 & $10(67 \%) / 5(33 \%)$ & $50.25 \pm 16.5$ & $\mathrm{NA}$ & $\begin{array}{l}\text { Deceased: } 12(80 \%) \\
\text { Living: } 3(20 \%)\end{array}$ & $\mathrm{NA}$ & $\begin{array}{l}\text { Tacrolimus: } 14(93 \%) \\
\text { Prednisone: } 10(67 \%) \\
\text { MMF/MPA: } 12(80 \%) \\
\text { Azathioprine: } 1(7 \%) \\
\text { Leflunomide: } 1(7 \%) \\
\text { Belatacept: } 2(13 \%)\end{array}$ & $\begin{array}{l}49(0-232) \text { months } \\
\text { (one recent transplant } \\
<3 \text { months) }\end{array}$ & NA \\
\hline Nair et al. [23] & United States & 10 & $6(60 \% / 4(40 \%)$ & $56.05 \pm 14.3$ & $\begin{array}{l}\text { African American: } 3(30 \%) \\
\text { Caucasian: } 4(40 \%) \\
\text { Asian: } 1(10 \%) \\
\text { Others: } 2(20 \%)\end{array}$ & $\begin{array}{l}\text { Deceased: } 4(40 \%) \\
\text { Living: } 5(50 \%) \\
\text { Unknown: } 1(10 \%)\end{array}$ & $\begin{array}{l}\text { HTN: } 10(100 \%) \\
\text { DM: } 8(80 \%) \\
\text { CAD: } 2(20 \%) \\
\text { Cancer 1 } 1(10 \%) \\
\text { Smoking history: } 2(20 \%)\end{array}$ & $\begin{array}{l}\text { Tacrolimus: } 9(90 \%) \\
\text { Prednisone : } 7(70 \%) \\
\text { MMF/MP: } 9(90 \%) \\
\text { mTORi: } 2(20 \%)\end{array}$ & $\begin{array}{l}94.0(4.1-248.0) \\
\text { months }\end{array}$ & $2.25 \pm 1.15$ \\
\hline $\begin{array}{l}\text { Fernandez-Ruiz et al. } \\
\text { [24] }\end{array}$ & Spain & 8 & $7(87 \%) / 1(13 \%)$ & $65.75 \pm 11.8$ & $\mathrm{NA}$ & $\mathrm{NA}$ & $\begin{array}{l}\text { HTN: } 8(100 \%) \\
\text { DM: 4(50\%) } \\
\text { CAD: } 1(12 \%) \\
\text { PAD 1 } 1(12 \%) \\
\text { Obesity: } 1(12 \%)\end{array}$ & 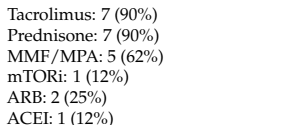 & $7.4(1.8-30.1)$ years & NA \\
\hline Zhu et al. [25] & China & 10 & $8(80 \%) / 2(20 \%)$ & $47 \pm 11.8$ & $\mathrm{NA}$ & $\mathrm{NA}$ & $\begin{array}{l}\text { HTN: } 5(50 \%) \\
\text { CAD } 3(12 \%) \\
\text { PAD: 1 (12\%) } \\
\text { Obesity: 1(12\%) }\end{array}$ & $\begin{array}{l}\text { Tacrolimus: } 9(90 \%) \\
\text { Cyclosporine: } 1(10 \%) \\
\text { Prednisone: } 7(70 \%) \\
\text { MMF /MPA: }(90 \%) \\
\text { Mizoribine: } 1(10 \%)\end{array}$ & $\mathrm{NA}$ & NA \\
\hline Cavagna et al. [26] & Italy & 6 & $5(83 \%) / 1(17 \%)$ & $57.5 \pm 3.8$ & $\mathrm{NA}$ & $\mathrm{NA}$ & NA & $\begin{array}{l}\text { Tacrolimus: } 3(50 \%) \\
\text { Cyclosporine: } 3(50 \%)\end{array}$ & $\mathrm{NA}$ & $\mathrm{NA}$ \\
\hline Fung et al. [27] & United States & 7 & $5(71 \%) / 2(29 \%)$ & $60.75 \pm 9.5$ & $\begin{array}{l}\text { African American: } 3(43 \%) \\
\text { Asian: 2 } 2(29 \%)) \\
\text { Hispanics: } 1(11 \%) \\
\text { Others: } 1(14 \%)\end{array}$ & $\begin{array}{l}\text { Deceased: } 4(57 \%) \\
\text { Living: } 3(43 \%)\end{array}$ & $\begin{array}{l}\text { HTN: } 4(57 \%) \\
\text { CAD: } 4(57 \%) \\
\text { DM: } 1(14 \%)\end{array}$ & $\begin{array}{l}\text { Tacrolimus: } 7(100 \%) \\
\text { Prednisone: } 6(86 \%) \\
\text { MMF/MPA: } 5(100 \%)\end{array}$ & $\begin{array}{l}2.9(0.3-10.8) \text { years } \\
4.2 \pm 3.0\end{array}$ & $154 \pm 0.75$ \\
\hline
\end{tabular}


Table A1. Cont.

\begin{tabular}{|c|c|c|c|c|c|c|c|c|c|c|}
\hline Study & Country & $\begin{array}{l}\text { Number of } \\
\text { Patients in the } \\
\text { Study }\end{array}$ & $\operatorname{Sex}(M / F)$ & Age (Years) & Ethnicity & Donor Type & Comorbidities & $\begin{array}{l}\text { Medications (Immunosuppression } \\
\text { + Others) }\end{array}$ & $\begin{array}{l}\text { Time Since } \\
\text { Renal-Transplant, } \\
\text { Median (Range) or } \\
\text { Mean } \pm \text { SD }\end{array}$ & $\begin{array}{l}\text { Baseline Serum } \mathrm{Cr} \text {, } \\
\text { Median (Range) mg/dL }\end{array}$ \\
\hline Abrishami et al. [28] & Iran & 12 & $9(75 \%) / 3(25 \%)$ & $47.5 \pm 10.7$ & $\mathrm{NA}$ & $\mathrm{NA}$ & $\begin{array}{l}\text { HTN: } 2(57 \%) \\
\text { CAD: } 4(57 \%) \\
\text { DM: } 1(14 \%)\end{array}$ & $\mathrm{NA}$ & $\begin{array}{l}13(3.0-18) \text { years } \\
11.8 \pm 4.3\end{array}$ & $2.14(0.9-4.6)$ \\
\hline Hoek et al. [29] & Netherland & $\begin{array}{l}16 \\
\text { (1 kidney with } \\
\text { heart }\end{array}$ & $11(69 \%) / 5$ & $56.0 \pm 17.3$ & $\mathrm{NA}$ & $\begin{array}{l}\text { Deceased: } 5(31 \%) \\
\text { Living: } 11(69 \%)\end{array}$ & $\mathrm{NA}$ & $\begin{array}{l}\text { CNI: } 16(100 \%) \\
\text { Steroid: } 3(18 \%) \\
\text { MMF/MPA: } 15(94 \%)\end{array}$ & $6(1.5-13)$ years & $\mathrm{NA}$ \\
\hline Mella et al. [30] & Italy & 6 & $6(100 \%) / 0(0 \%)$ & $55.5 \pm 6.9$ & $\mathrm{NA}$ & $\begin{array}{l}\text { Deceased: } 2(33 \%) \\
\text { Living: } 0(0 \%) \\
\text { Unknown: } 4(67 \%)\end{array}$ & $\begin{array}{l}\text { HTN: } 4(67 \%) \\
\text { CAD: } 5(83 \%) \\
\text { DM: } 1(17 \%)\end{array}$ & $\begin{array}{l}\text { CNI: } 6(100 \%) \\
\text { Steroid: 6(100\%) } \\
\text { MMF: } 3(50 \%)\end{array}$ & $10.7(0.02-14.7)$ years & $\mathrm{NA}$ \\
\hline $\begin{array}{l}\text { Montagud-Marrahi } \\
\text { et al. [31]] }\end{array}$ & Spain & $\begin{array}{l}33 \\
(2 \mathrm{KP})\end{array}$ & $19(57.6 \%) / 14(42.4 \%)$ & $57.3 \pm 17$ & $\mathrm{NA}$ & $\mathrm{NA}$ & $\mathrm{NA}$ & $\begin{array}{l}\text { CNIs: } 29(87.8 \%) \\
\text { Prednisone: } 26(78.8 \%) \\
\text { MMF/MPA: } \\
\text { mTORi: } 14(42.4 \% 2.5 \%)\end{array}$ & $10.7(4-14.7)$ years & $\mathrm{NA}$ \\
\hline Banerjee et al. [32] & UK & 7 & $4(57 \%) / 3(43 \%)$ & $55.5 \pm 6.9$ & $\mathrm{NA}$ & $\begin{array}{l}\text { Deceased: } 5(71 \%) \\
\text { Living : N } \mathrm{N} \\
\text { (No details available in } 2 \\
\text { cases, hal has second } \\
\text { transplant) }\end{array}$ & $\begin{array}{l}\text { HTN: } 6(86 \%) \\
\text { DM: } 3(43 \%)\end{array}$ & 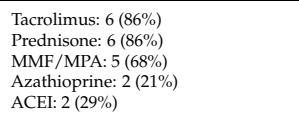 & $\begin{array}{l}\text { (01-360) months } \\
\text { NA } \\
(2 \text { in } \leq 3 \text { months) }\end{array}$ & $2.04(1.5-5.1)$ \\
\hline Malberti et al. [33] & Italy & 11 & $10(91 \%) / 1(9 \%)$ & $58.5 \pm 11$ & $\mathrm{NA}$ & $\mathrm{NA}$ & $\begin{array}{l}\text { HTN: NA } \\
\text { DM: } 1(9.1 \%) \\
\text { CAD: } 0(0 \%) \\
\text { Cancer: } 0(0 \%)\end{array}$ & $\begin{array}{l}\text { Tacrolimus: } 11(100 \%) \\
\text { MMF: } 11(100 \%)\end{array}$ & $\mathrm{NA}$ & $\mathrm{NA}$ \\
\hline Lubetzky et al. [34] & United States & 39 & $31(79 \%) / 8(21 \%)$ & $57.5 \pm 15.5$ & $\begin{array}{l}\text { African American: } 10 \\
\text { (26\%) } \\
\text { Hispanics: } 14(36 \%) \\
\text { Cacuasaina: } 1(128 \%) \\
\text { Asian: } 4(10 \%)\end{array}$ & $\begin{array}{l}\text { Deceased: } 13(33 \%) \\
\text { Living: } 26(67 \%)\end{array}$ & $\begin{array}{l}\text { HTN : } 37(95 \%) \\
\text { DM: } 12(31 \%) \\
\text { CAD: } 16(141 \%) \\
\text { Lung disease } 7(18 \%) \\
\text { Smoking: } 8(21 \%)\end{array}$ & $\begin{array}{l}\text { Tacrolimus: } 37(95 \%) \\
\text { Prednisone: } 36(92 \%) \\
\text { MMF /MPA: } 38(97 \%) \\
\text { ACEI: } 12(32 \%)\end{array}$ & $4.7(0.3-14.4)$ years & $1.58 \pm 0.74$ \\
\hline Devresee et al. [35] & Belgium & 18 & $\begin{array}{l}8(44 \%) / \\
10(56 \%)\end{array}$ & $54.5 \pm 6.3$ & $\begin{array}{l}\text { African American: } 6(33 \%) \\
\text { Caucasian: 11(61\%) } \\
\text { Asian: } 1(6 \%)\end{array}$ & $\mathrm{NA}$ & $\begin{array}{l}\text { HTN: } 14(78 \%) \\
\text { DM: } 4(22 \%) \\
\text { CAD: } 4(22 \%) \\
\text { Cancer: } 1(6 \%) \\
\text { Obesity: } 4(22 \%)\end{array}$ & $\begin{array}{l}\text { Tacrolimus: } 10(55 \%) \\
\text { Cyclosporine: } 5(27 \%) \\
\text { Prednisone: } 18(100 \%) \\
\text { MMF/MPA: } 1(26 \%) \\
\text { mTTR: } 3(17 \%) \\
\text { Azathioprine: } 3(17 \%) \\
\text { ACEI/ARB: } 10(56 \%)\end{array}$ & $\begin{array}{l}89 \text { (1-402) months } \\
\text { (3 in } \leq 3 \text { months) }\end{array}$ & $\mathrm{NA}$ \\
\hline Crespo et al. [36] & Spain & 414 & $265(64 \%) / 149(36 \%)$ & $62 \pm 3.17$ & $\mathrm{NA}$ & $\mathrm{NA}$ & $\mathrm{NA}$ & $\begin{array}{l}\text { CNIs: } 338(82 \%) \\
\text { Predisone } 310(75 \%) \\
\text { MMF /MPA: } 297(50 \%) \\
\text { mTORi: } 94(23 \%)\end{array}$ & 72 (31-145) months & $\mathrm{NA}$ \\
\hline Fava et al. [37] & Spain & 89 & $56(63 \%) / 33(37 \%)$ & $59.7 \pm 13.4$ & $\begin{array}{l}\text { African American: } 4(5 \%) \\
\text { Hispanic: } 6(7 \%)) \\
\text { Cauccasian: } 78(88 \%) \text { Asian: } \\
1(1 \%)\end{array}$ & $\begin{array}{l}\text { Deceased: } 75(84 \%) \\
\text { Living: } 14(16 \%)\end{array}$ & $\begin{array}{l}\text { HTN: } 77(87 \%) \\
\text { DM: } 25(28 \%) \\
\text { CAD: } 25(28 \%) \\
\text { Lung disease: } 14(16 \%) \\
\text { Obesity: } 24(27 \%) \\
\text { Cancer: } 7(8 \%) \\
\text { Smoking: } 8(50 \%)\end{array}$ & $\begin{array}{l}\text { CNIs: } 78(88 \%) \\
\text { Prednisone: } 81(91 \%) \\
\text { MMF/MPA: } 73(82 \%) \\
\text { mTORi: } 18(20 \%) \\
\text { ACEI/ARB: } 33(37 \%)\end{array}$ & $\begin{array}{l}4.7 \text { (NA) years } \\
(11 \text { in } \leq 6 \text { months })\end{array}$ & $1.8 \pm 0.8$ \\
\hline Zhang et al. [38] & China & 5 & $4(80 \%) / 1(20 \%)$ & $45.0 \pm 11$ & $\mathrm{NA}$ & $\mathrm{NA}$ & $\begin{array}{l}\text { HTN: } 2(40 \%) \\
\text { DM: } 1(20 \%) \\
\text { Cancer: } 1(20 \%)\end{array}$ & $\begin{array}{l}\text { CNIs: } 5(100 \%) \\
\text { Prednisone: } 5(100 \%) \\
\text { MMF/MPA: } 5(100 \%)\end{array}$ & $1.53 \pm 1.2$ years & $\mathrm{NA}$ \\
\hline Cravedi et al. [39] & Multicentric & 144 & $94(65.3 \%) / 50(34.7 \%)$ & $61.25 \pm 4.90$ & 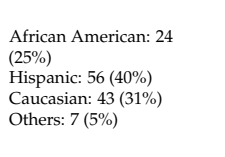 & $\begin{array}{l}\text { Deceased: } 112(78 \%) \\
\text { Living: } 32(22 \%)\end{array}$ & $\begin{array}{l}\text { HTN: } 137(95 \%) \\
\text { DM: } 75(52 \%) \\
\text { CAD: } 41(29 \%) \\
\text { Lung disease: } 27(19 \%) \\
\text { Obesity: } 71 \text { (50\%) } \\
\text { Cancer: } 22(15 \%) \\
\text { Smoking: 39 (29\%) }\end{array}$ & $\begin{array}{l}\text { Tacrolimus: } 131 \text { (91\%) } \\
\text { Prednisone: } 125(87 \%) \\
\text { mTORi: } 11(7.6 \%) \text { MMF /MPA: } 111 \\
\text { (77\%) } \\
\text { ARB: } 24(17 \%) \\
\text { ACEI: } 20(14 \%)\end{array}$ & $5.31 \pm 2.1$ years & $1.5 \pm 0.30$ \\
\hline
\end{tabular}


Table A1. Cont.

\begin{tabular}{|c|c|c|c|c|c|c|c|c|c|c|}
\hline Study & Country & $\begin{array}{l}\text { Number of } \\
\text { Patients in the } \\
\text { Study }\end{array}$ & $\operatorname{Sex}(M / F)$ & Age (Years) & Ethnicity & Donor Type & Comorbidities & $\begin{array}{l}\text { Medications (Immunosuppression } \\
\text { + Others) }\end{array}$ & $\begin{array}{l}\text { Time Since } \\
\text { Renal-Transplant, } \\
\text { Median (Range) or } \\
\text { Mean } \pm \text { SD }\end{array}$ & $\begin{array}{l}\text { Baseline Serum } \mathrm{Cr}_{r} \\
\text { Median (Range) mg/dL }\end{array}$ \\
\hline Maritati et al. [40] & Italy & 5 & $3(60 \%) / 2(40 \%)$ & $66.00 \pm 9.27$ & $\mathrm{NA}$ & $\begin{array}{l}\text { Deceased: } 4(80 \%) \\
\text { Living: } 1(20 \%)\end{array}$ & $\begin{array}{l}\text { HTN: } 5(100 \%) \\
\text { DM: } 1(20 \%) \\
\text { CAD: 2(40\%) } \\
\text { Obesity: } 2(40 \%)\end{array}$ & $\begin{array}{l}\text { Tacrolimus: } 5(100 \%) \\
\text { Prednisone: 5(100\%\%) } \\
\text { mTOR: } 120 \%) \\
\text { MMF/MPA: } 4(80 \%) \\
\end{array}$ & $4.20 \pm 3.1$ years & $1.85 \pm 0.80$ \\
\hline Elias et al. [41] & France & 66 & $37(56 \%) / 29(44 \%)$ & $56.40 \pm 12.5$ & $\begin{array}{l}\text { Caucasian: } 42(64 \%) \\
\text { Others: } 24(36 \%)\end{array}$ & $\begin{array}{l}\text { Deceased: } 64(97 \%) \\
\text { Living: } 2(3 \%)\end{array}$ & $\mathrm{NA}$ & $\begin{array}{l}\text { Tacrolimus: } 57(86 \%) \\
\text { Prednisone: } 55(83 \%) \\
\text { MMF/MPA: } 61(92 \%) \\
\text { Belatacept: } 6(9 \%)\end{array}$ & $4.81 \pm 3.9$ years & $\mathrm{NA}$ \\
\hline Chen et al. [42] & United States & 30 & $16(53 \%) / 14(37 \%)$ & $56 \pm 12$ & $\begin{array}{l}\text { African American: } 22 \\
\text { (73\%) } \\
\text { Hispanic: } 5(17 \%) \\
\text { Cacuasian: } 2(7 \%)) \\
\text { Asian: } 1(3 \%)\end{array}$ & $\begin{array}{l}\text { Deceased: } 18(60 \%) \\
\text { Living: } 12(40 \%)\end{array}$ & $\begin{array}{l}\text { HTN: } 29(97 \%) \\
\text { DM: } 14(74 \%) \\
\text { CAD: 11(37\%) } \\
\text { Lung disease: } 0(0 \%) \\
\text { Obesity: } 10(33 \%)\end{array}$ & $\begin{array}{l}\text { CNIs: } 29 \text { (97\%) } \\
\text { Prednisone: } 30(100 \%) \\
\text { MMF/MPA: } 12(40 \%)\end{array}$ & $8.4 \pm 2.9$ years & $1.9 \pm 0.42$ \\
\hline Mehta et al. [43] & United States & 34 & $22(65 \%) / 12(45 \%)$ & $58.6 \pm 3.25$ & $\begin{array}{l}\text { African American: } 15 \\
\text { (44\%) } \\
\text { Hispanic: } 8(23 \%) \\
\text { Cacucasian: } 7(20 \%) \\
\text { Asian: } 2(7 \%)\end{array}$ & $\begin{array}{l}\text { Deceased: } 27(79 \%) \\
\text { Living: } 7(21 \%)\end{array}$ & $\mathrm{NA}$ & 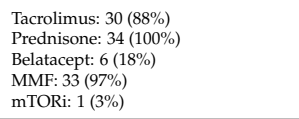 & $1.21 \pm 0.56$ years & $\mathrm{NA}$ \\
\hline Silva et al. [44] & Portugal & 5 & $3(75 \%) / 2(25 \%)$ & $50.4 \pm 14.4$ & $\mathrm{NA}$ & $\begin{array}{l}\text { Deceased: } 4(80 \%) \\
\text { Living: } 1(20 \%)\end{array}$ & $\begin{array}{l}\text { HTN: } 5(100 \%) \\
\text { DM: } 2(40 \%) \\
\text { CAD: } 1(20 \%) \\
\text { Lung disease: } 1(20 \%) \\
\text { Obesity: } 3(60 \%) \\
\text { Smoking: } 1(20 \%)\end{array}$ & $\begin{array}{l}\text { CNI: } 5(100 \%) \\
\text { Prednisone: } 5(100 \%) \\
\text { MMF: } 3(66 \%) \\
\text { Azathioprine: } 2(40 \%)\end{array}$ & $5.1 \pm 5.9$ years & $1.6 \pm 0.42$ \\
\hline Chavarot et al. [45] & France & 100 & $64(64 \%) / 36(36 \%)$ & $64.45 \pm 5.13$ & $\mathrm{NA}$ & $\mathrm{NA}$ & $\begin{array}{l}\text { HTN: } 85(85 \%) \\
\text { DM: } 84(88 \%) \\
\text { CAD: } 18(18 \%) \\
\text { Lung disease: } 89(89 \%)\end{array}$ & 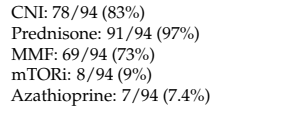 & $6.4 \pm 2.9$ years & $1.3 \pm 0.26$ \\
\hline Meester et al. [46] & $\begin{array}{l}\text { US and European } \\
\text { countries }\end{array}$ & 46 & $26(56 \%) / 20(44 \%)$ & $\mathrm{NA}$ & $\mathrm{NA}$ & $\mathrm{NA}$ & $\mathrm{NA}$ & $\mathrm{NA}$ & $\mathrm{NA}$ & $\mathrm{NA}$ \\
\hline Abolghasemi et al. [47] & Iran & 24 & $15(62.5 \%) / 9(37.5 \%)$ & $47.75 \pm 10.09$ & $\mathrm{NA}$ & $\begin{array}{l}\text { Deceased: } 18(75 \%) \\
\text { Living: }(25 \%)\end{array}$ & $\begin{array}{l}\text { HTN: } 15(62.5 \%) \\
\text { DM: } 5(20 \%)(2 \%) \\
\text { CAD: (417\%) } \\
\text { Lung disease: } 1(4 \%)\end{array}$ & $\begin{array}{l}\text { CNI: } 22 \text { (92\%) } \\
\text { Prednisone: } 24(10 \% \%) \\
\text { MMF: 20 (83\%) } \\
\text { mTORi: } 1(4 \%) \\
\text { Azathioprine: } 2(8 \%)\end{array}$ & $10.3(1-20)$ years & $\mathrm{NA}$ \\
\hline Cucchiariet al. [48] & Spain & 28 & $18(64 \%) / 10(36 \%)$ & $54.81 \pm 6.3$ & $\mathrm{NA}$ & $\mathrm{NA}$ & $\mathrm{NA}$ & $\begin{array}{l}\text { CNI: } 23(82 \%) \\
\text { MPA: } 14(50 \%) \\
\text { mTORi: } 9(32 \%)\end{array}$ & $5.4 \pm 2.5$ years & $1.60 \pm 0.36$ \\
\hline Kute et al. [49] & India & 250 & $215(86 \%) / 35(14 \%)$ & $43.00 \pm 4.6$ & $\mathrm{NA}$ & $\begin{array}{l}\text { Deceased: } 24(9.6 \%) \\
\text { Living: } 226(90 \%)\end{array}$ & $\begin{array}{l}\text { HTN: } 210(84 \%) \\
\text { DM: } 80(32 \%) \\
\text { CAD: } 30(12 \%) \\
\text { Obesity: } 53(24 \%)\end{array}$ & $\begin{array}{l}\text { CNI: } 236(94 \%) \\
\text { Prednisone: } 250(100 \%) \\
\text { MMF: } 250(100 \%) \\
\text { mTORi: } 14(5.6 \%)\end{array}$ & $3.75 \pm 1.29$ years & $1.88 \pm 0.42$ \\
\hline Caillard et al. [50] & France & 243 & $162(67 \%) / 81(33 \%)$ & $60.75 \pm 5.25$ & $\mathrm{NA}$ & $\mathrm{NA}$ & $\begin{array}{l}\text { HTN: } 201(83 \%) \\
\text { DMA : } 22(39 \%) \\
\text { CAD: } 81(3 \% \%) \\
\text { Cancer } 35 \text { (114\%) } \\
\text { Smoking: } 30(12 \%)\end{array}$ & $\begin{array}{l}\text { CNI: } 202(83 \%) \\
\text { Prednisone: } 177(73 \%) \\
\text { MMF: } 183(75 \%) \\
\text { mTORi: } 29(12 \%) \\
\text { Belatacept: } 15(6.1 \%)\end{array}$ & $6.08 \pm 2.26$ years & $2.05 \pm 0.42$ \\
\hline Kutzler et al. [51] & United States & 10 & $\mathrm{NA}$ & $62.60 \pm 7.00$ & $\begin{array}{l}\text { African American: } 2(20 \%) \\
\text { Hispanic: } 3(30 \%) \\
\text { Caucasian: } 5(50 \%)\end{array}$ & $\mathrm{NA}$ & $\begin{array}{l}\text { HTN: } 8(80 \%) \\
\text { DM: } 5(50 \%) \\
\text { CAD: 6(60\%) } \\
\text { Cancer: 2 2(20\%) } \\
\text { Obesity: 2(20\%) }\end{array}$ & $\begin{array}{l}\text { CNI: } 5(50 \%) \\
\text { Prednisone: } 7(70 \%) \\
\text { MMFF:5 (50\%) } \\
\text { mTTR: } 1 \text { (110\%) } \\
\text { Belatacept: 10(10\%) }\end{array}$ & $10.98 \pm 8.7$ years & $\mathrm{NA}$ \\
\hline Mairons et al. [52] & Spain & 30 & $\mathrm{NA}$ & $\mathrm{NA}$ & $\mathrm{NA}$ & $\mathrm{NA}$ & $\mathrm{NA}$ & $\mathrm{NA}$ & $\mathrm{NA}$ & $\mathrm{NA}$ \\
\hline Sharma et al. [53] & United States & 16 & $\mathrm{NA}$ & $\mathrm{NA}$ & $\mathrm{NA}$ & $\mathrm{NA}$ & $\mathrm{NA}$ & $\mathrm{NA}$ & $\mathrm{NA}$ & $\mathrm{NA}$ \\
\hline
\end{tabular}


Table A1. Cont.

\begin{tabular}{|c|c|c|c|c|c|c|c|c|c|c|}
\hline Study & Country & $\begin{array}{l}\text { Number of } \\
\text { Patients in the } \\
\text { Study }\end{array}$ & $\operatorname{Sex}(M / F)$ & Age (Years) & Ethnicity & Donor Type & Comorbidities & $\begin{array}{l}\text { Medications (Immunosuppression } \\
\text { + Others) }\end{array}$ & $\begin{array}{l}\text { Time Since } \\
\text { Renal-Transplant, } \\
\text { Median (Range) or } \\
\text { Mean } \pm \text { SD }\end{array}$ & $\begin{array}{l}\text { Baseline Serum } \mathrm{Cr} \\
\text { Median (Range) } \mathrm{mg} / \mathrm{dL}\end{array}$ \\
\hline Mohamed et al. [54] & United Kingdom & 35 & $16(57 \%) / 11(43 \%)$ & $52.75 \pm 13.55$ & $\mathrm{NA}$ & $\begin{array}{l}\text { Deceased: } 22(79 \%) \\
\text { Living: } 13(21 \%)\end{array}$ & $\begin{array}{l}\text { HTN: } 23(85 \%) \\
\text { DM: } 1(37 \%) \\
\text { CAD: } 5(18 \%) \\
\text { Obesity: } 181(75 \%) \\
\text { Lung disease: } 4(14 \%)\end{array}$ & $\begin{array}{l}\text { CNI: } 27 / 27(100 \%) \\
\text { Prednisone: } 27 / 27(100 \%) \\
\text { MMFF:21/27 (78\%) } \\
\text { Azathioprine: } 3 / 27 \text { (10\%) }\end{array}$ & $6.38 \pm 5.43$ years & $2.08 \pm 0.97$ \\
\hline Felldin et al. [55] & Sweden & 22 & $18(82 \%) / 4(18 \%)$ & $54.11 \pm 11.26$ & $\mathrm{NA}$ & $\mathrm{NA}$ & $\begin{array}{l}\text { HTN: } 16(73 \%) \\
\text { DM: 7 (32\%) } \\
\text { CAD: 2(2\%) } \\
\text { Lundisease: } 2(9 \%) \\
\text { Obesity: } 10(45 \%) \\
\end{array}$ & $\begin{array}{l}\text { CNI: } 22 \text { (100\%) } \\
\text { Prednisone: } 23(57.5 \%) \\
\text { MMF: } 12 \text { (55\%) } \\
\text { Azathioprine: } 2 \text { (9\%) }\end{array}$ & $10.99 \pm 1.74$ years & $\mathrm{NA}$ \\
\hline Kates et al. [56] & United States & 318 & $\mathrm{NA}$ & $\mathrm{NA}$ & $\mathrm{NA}$ & $\mathrm{NA}$ & $\mathrm{NA}$ & NA & $\mathrm{NA}$ & $\mathrm{NA}$ \\
\hline Benotmane et al. [57] & France & 40 & $31(77.5 \%) / 9(22.5 \%)$ & $63.8 \pm 3.4$ & $\mathrm{NA}$ & $\mathrm{NA}$ & $\begin{array}{l}\text { HTN: } 33(82.5 \%) \\
\text { DM: } 19(47.5 \%) \\
\text { CAD: } 16(40 \%) \\
\text { Lung disease: } 9 \text { (22.5\%) } \\
\text { Obesity: } 20(50 \%)\end{array}$ & $\begin{array}{l}\text { CNI: } 35(87.5 \%) \\
\text { Prednisone: } 23(57.5 \%) \\
\text { MMF: } 34 \text { (85\%) } \\
\text { Azathioprine: } 1(2.5 \%)\end{array}$ & $\mathrm{NA}$ & \\
\hline Chaudhry et al. [58] & United States & 38 & $26(68 \%) / 12(32 \%)$ & $62 \pm 5.75$ & $\begin{array}{l}\text { African American: } 31 \\
(81.5 \%)\end{array}$ & $\mathrm{NA}$ & $\begin{array}{l}\text { HTN: } 37(97 \%) \\
\text { DM: } 27(71 \%) \\
\text { CAD: } 11(29 \%) \\
\text { Lung disease: } 5(13 \%) \\
\text { Smoking: } 7(18 \%) \\
\end{array}$ & $\mathrm{NA}$ & $\mathrm{NA}$ & $\mathrm{NA}$ \\
\hline $\begin{array}{l}\text { Craig-Schapiro et al. } \\
{[599]}\end{array}$ & United States & 80 & $56(70 \%) / 24(30 \%)$ & $57 \pm 9.16$ & $\begin{array}{l}\text { African American: } 21 \\
\text { (26\%) } \\
\text { Hispanic: 24 } 2430 \%) \\
\text { Cacuacaian: } 25(3131 \%) \\
\text { Asian } 9(11 \%)\end{array}$ & $\mathrm{NA}$ & $\begin{array}{l}\text { HTN: } 20(25 \%) \\
\text { DM: } 22(27 \%) \\
\text { CAD: 22(62\%) } \\
\text { Lung disease: 11 114\%) } \\
\text { Smoking: 16 (20\%) }\end{array}$ & $\begin{array}{l}\text { Prednisone: } 22(51 \%) \\
\text { MMF: } 52(47 \%)\end{array}$ & $\mathrm{NA}$ & $1.3 \pm 0.22$ \\
\hline Hilbrands et al. [61] & $\begin{array}{l}\text { ERACODA } \\
\text { Collaborators, Europe }\end{array}$ & 271 & $118(43.5 \%) / 153(56.5 \%)$ & $60 \pm 12$ & $\begin{array}{l}\text { African American: } 15 \\
(0.06 \%) \\
\text { Caucasian: } 151(56 \%) \\
\text { Asian } 7(3 \%)\end{array}$ & $\mathrm{NA}$ & $\begin{array}{l}\text { HTN: } 172(63 \%) \\
\text { DM: } 64(24 \%) \\
\text { CAD: } 37(14 \%) \\
\text { Lung disease: } 19(7 \%) \\
\text { Cancer: } 16(6 \%) \\
\text { Obesity: } 55(20 \%)\end{array}$ & $\begin{array}{l}\text { CNI: } 175(65 \%) \\
\text { Prednisone: } 173(64 \%) \\
\text { MMF: } 145(54 \%) \\
\text { mTORi: } 33(12 \%)\end{array}$ & $\mathrm{NA}$ & $\mathrm{NA}$ \\
\hline Pascual et al. [62] & Spain & 24 & $9(37.5 \%) / 15(62.5 \%)$ & $62 \pm 8.75$ & $\mathrm{NA}$ & $\begin{array}{l}\text { Deceased: } 23(96 \%) \\
\text { Living: } 1(4 \%)\end{array}$ & $\begin{array}{l}\text { HTN: } 22(92 \%) \\
\text { DM: } 12(50 \%)\end{array}$ & $\begin{array}{l}\text { CNI: } 24(100 \%) \\
\text { Prednisone: } 24(100 \%) \\
\text { MMF: } 21(88 \%) \\
\text { mTORi: } 2(8 \%)\end{array}$ & $\mathrm{NA}$ & $\mathrm{NA}$ \\
\hline $\begin{array}{l}\text { Rodriguez-Cubillo } \\
\text { et al. [63] }\end{array}$ & Spain & 29 & $17(58.6 \%) / 12(41.4 \%)$ & $66 \pm 3.25$ & $\mathrm{NA}$ & $\mathrm{NA}$ & $\begin{array}{l}\text { HTN: } 28(97 \%) \\
\text { DM: } 2(40 \%) \\
\text { Obesity: } 15(52 \%)\end{array}$ & $\begin{array}{l}\text { CNI: } 24(83 \%) \\
\text { Prednisone: } 23 \text { (79\%) } \\
\text { MMF: 23 (79\%) }\end{array}$ & $\mathrm{NA}$ & $\mathrm{NA}$ \\
\hline $\begin{array}{l}\text { Sanchez-Alvarez et al. } \\
\text { [64] }\end{array}$ & Spain & 470 & $299(63.6 \%) / 171(36.4 \%)$ & $60 \pm 13$ & $\mathrm{NA}$ & $\mathrm{NA}$ & $\mathrm{NA}$ & $\mathrm{NA}$ & $\mathrm{NA}$ & $\mathrm{NA}$ \\
\hline
\end{tabular}


Table A1. Cont.

\begin{tabular}{|c|c|c|c|c|c|c|c|c|c|c|}
\hline Study & Country & $\begin{array}{l}\text { Number of } \\
\text { Patients in the } \\
\text { Study }\end{array}$ & $\operatorname{Sex}(M / F)$ & Age (Years) & Ethnicity & Donor Type & Comorbidities & $\begin{array}{l}\text { Medications (Immunosuppression } \\
\text { + Others) }\end{array}$ & $\begin{array}{l}\text { Time Since } \\
\text { Renal-Transplant, } \\
\text { Median (Range) or } \\
\text { Mean } \pm \text { SD }\end{array}$ & $\begin{array}{l}\text { Baseline Serum Cr, } \\
\text { Median (Range) mg/dL }\end{array}$ \\
\hline Oto et al. [65] & Turkey & 109 & $63(57.8 \%) / 46(42.2 \%)$ & $48.4 \pm 12.4$ & $\mathrm{NA}$ & $\begin{array}{l}\text { Deceased: } 17(16 \%) \\
\text { Living: } 92(84 \%)\end{array}$ & $\begin{array}{l}\text { HTN: } 81 / 106(76 \%) \\
\text { DM: } 25 / 107(23 \%) \\
\text { CAD: } 18 / 103(17 \%) \\
\text { Lung disease: } 5 / 105(5 \%) \\
\text { Cancer: } 6 / 105(6 \%) \\
\text { Smoking: } 23(21 \%)\end{array}$ & $\begin{array}{l}\text { CNI: } 95(87 \%) \\
\text { Prednisone: } 106(97 \%) \\
\text { MMFF: } 94 \text { (866) } \\
\text { mTORi: } 12(11 \%)\end{array}$ & $5.0(3.0-9.0)$ years & $1.5(1.10-2.19)$ \\
\hline Elhadedy et al. [66] & United Kingdom & 8 & $4(50 \%) / 4(50 \%)$ & $50.14 \pm 17.5$ & $\mathrm{NA}$ & $\mathrm{NA}$ & $\begin{array}{l}\text { HTN: } 4 / 8(50 \%) \\
\text { DM: } 1 / 8(13 \%) \\
\text { Lung disease: } 1 / 8(13 \%)\end{array}$ & $\begin{array}{l}\text { CNI: } 8 / 8(100 \%) \\
\text { Prednisone: 8/8 } \\
\text { MMF: } 7 / 8 \text { (88\%) }\end{array}$ & $4.6 \pm 4.8$ years & $3.23 \pm 163.60$ \\
\hline
\end{tabular}

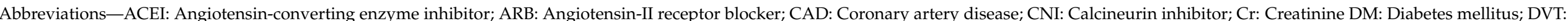

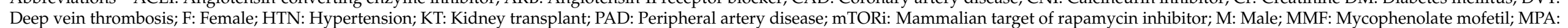
Mycophenolic acid; NA: Not available/Not applicable. 
Table A2. Clinical and laboratory data of post-kidney transplant patients with COVID-19.

\begin{tabular}{|c|c|c|c|c|c|c|c|}
\hline \multirow{2}{*}{ Study } & \multirow{2}{*}{ Clinical Presentation } & \multirow{2}{*}{ Respiratory Disease Severity } & \multirow{2}{*}{$\begin{array}{l}\text { Intensive Care Unit Admission \& } \\
\text { Organ Failure (Respiratory or Renal) }\end{array}$} & \multicolumn{2}{|l|}{ Hematological Data } & \multicolumn{2}{|l|}{ Serum Markers } \\
\hline & & & & Median (Range) & Significant Decline $n(\%)$ & Median (Range) & Significant Change $n(\%)$ \\
\hline Akalin et al. [19] & $\begin{array}{l}\text { Fever: } 21(58 \%) \\
\text { Dyspnea: } 16(44 \%) \\
\text { Cought } 19(53 \%) \\
\text { GIs: } 8(22 \%)\end{array}$ & $\begin{array}{l}\text { Major: } 28(78 \%) \\
\text { Minor } 8 \text { (22\%) Outpatient } \\
\text { Radiographicevidence of pneumonia: } \\
27 / 28(96 \%)\end{array}$ & $\begin{array}{l}\text { ICU: } 11 / 28(39 \%) \\
\text { MV: 11/28 (39\%) } \\
\text { AKII NA } \\
\text { RRT: NA }\end{array}$ & 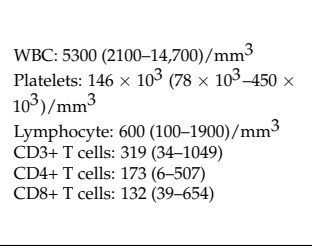 & 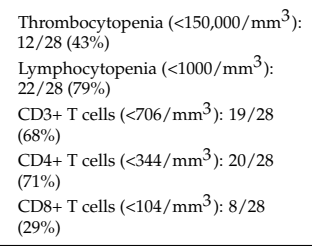 & $\begin{array}{l}\text { CRP: } 7.9(0.5-48.7) \mathrm{mg} / \mathrm{dL} \\
\text { Ferritin: } 1230(191-9259) \mathrm{ng} / \mathrm{mL} \\
\text { Procacitonini } 0.2(0.1-1.51) \mathrm{ng} / \mathrm{mL} \\
\text { D-dimer: } 1.02(0.32-5.19) \mu \mathrm{mg} / \mathrm{mL}\end{array}$ & 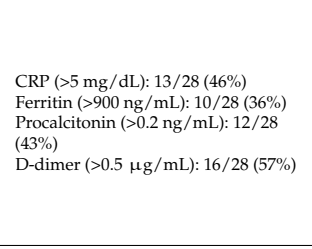 \\
\hline Bossini et al. [20] & $\begin{array}{l}\text { Fever: } 51(96 \%) \\
\text { Dyspea: } 15(28 \%) \\
\text { Cough: } 26(49 \%) \\
\text { Pharyngitis: } 7(13 \%) \\
\text { Diarrhea: } 9(17 \%)\end{array}$ & $\begin{array}{l}\text { Hospitalized: } 45(85 \%) \\
\text { Radiological evidence of pneumonia: } \\
38 / 39(97 \%)\end{array}$ & $\begin{array}{l}\text { ICU: } 10 / 45(22 \%) \\
\text { ARDS: } 27 / 45(60 \%) \\
\text { MV: } \\
9 / 45(20 \%) \\
\text { AKR: } 15 / 45(33 \%) \\
\text { RRT: } 3 / 15(20 \%)\end{array}$ & $\begin{array}{l}\text { WBC: } 5560(4140-7400) / \mathrm{mm}^{3} \\
\text { Platelets: } 162 \times 10^{3}\left(129 \times 10^{3}-219 \times\right. \\
\text { 10 } \\
\text { Lymphocyte: } 590(430-1092) / \mathrm{mm}^{3}\end{array}$ & NA & $\begin{array}{l}\text { CRP: } 3.9(1.6-10.3) \mathrm{mg} / \mathrm{dL} \\
\text { Ferritin: } 333(284-872) \mathrm{ng} / \mathrm{mL} \\
\text { D-dimer: } 0.41(0.10-0.68) \mu \mathrm{mg} / \mathrm{mL}\end{array}$ & NA \\
\hline Trujillo et al. [21] & $\begin{array}{l}\text { Fever: } 12(46 \%) \\
\text { Dyspnea: } 15(58 \%) \\
\text { Cought } 23(83 \%) \\
\text { GIs: } 10(38 \%)\end{array}$ & 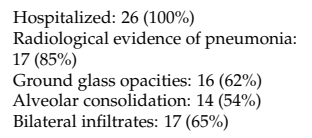 & $\begin{array}{l}\text { ICU: NA } \\
\text { ARDS: } 10 / 26(39 \%) \\
\text { MV NA } \\
\text { AKI: NA }\end{array}$ & Lymphocyte: 700 (400-1100) & NA & $\begin{array}{l}\text { CRP: } 13(6-23) \mathrm{mg} / \mathrm{dL} \\
\text { D-dimer: } 0.82(0.51-0.12) \mu \mathrm{gg} / \mathrm{mL}\end{array}$ & NA \\
\hline Columbia University KT program [22] & $\begin{array}{l}\text { Fever: } 13(87 \%) \\
\text { Dyspnea: } 4(27 \%) \\
\text { Cough: } 9(60 \%) \\
\text { Diarrhea: } 3(20 \%)\end{array}$ & $\begin{array}{l}\text { Hospitalized: } 15(100 \%) \\
\text { Radidological evidence of pneumonia: } \\
10 \text { (6l7\%) } \\
\text { Multifocal/bilateral patchy opacities: } \\
7(47 \% \%)\end{array}$ & $\begin{array}{l}\text { ICU: } 4 / 15(27 \%) \\
\text { MV: } 4 / 15(27 \%) \\
\text { AKI } 6 / 15(140 \%) \\
\text { RRT: } 2 / 6(33 \%)\end{array}$ & $\begin{array}{l}\text { WBC: } 4800(2100-12,700) / \mathrm{mm}^{3} \\
\text { Lymphocyte: } 800(110-1410) / \mathrm{mm}^{3}\end{array}$ & $\mathrm{NA}$ & 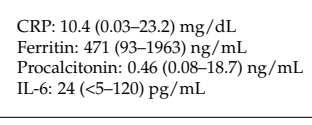 & $\mathrm{NA}$ \\
\hline Fernandez-Ruiz et al. [24] & $\begin{array}{l}\text { Fever: } 7(87 \%) \\
\text { Dyspnea: } 5(62 \%) \\
\text { Cough: } 4(50 \%) \\
\text { GIs: } 1(12 \%)\end{array}$ & $\begin{array}{l}\text { Hospitalized: } 8(100 \%) \\
\text { Radiological evidence of pneumonia: } \\
5 / 8(62 \%)\end{array}$ & $\begin{array}{l}\text { ICU: } 1 / 8(12 \%) \\
\text { ARDS: } 3 / 8(38 \%) \\
\text { CPAP } 38(37 \%) \\
\text { AKI: } 1 / 8(12 \%) \\
\text { RRT: } 1 / 1(100 \%) \\
\end{array}$ & NA & $\mathrm{NA}$ & $\mathrm{NA}$ & NA \\
\hline Zhu et al. [25] & $\begin{array}{l}\text { Fever: } 9(90 \%) \\
\text { Dyspnea: } 9(90 \%) \\
\text { Cough } 9(90 \%) \\
\text { GIs: } 3(30 \%)\end{array}$ & $\begin{array}{l}\text { Hospitalized: } 10(100 \%) \\
\text { Radiological evidence of pneumonia: } \\
10 \text { (1000\%) } \\
\text { B/L multiple consolidation: } 8\end{array}$ & $\begin{array}{l}\text { ICU: } 1 / 10(10 \%) \\
\text { OXygen requirenent: } \\
\text { NIVI:3/10 (30\%) } \\
\text { AKI: } 6110(60 \%) \\
\text { RRT: } 1 / 6(16 \%)\end{array}$ & Lymphocyte: 450 (190-990) / $\mathrm{mm}^{3}$ & $\begin{array}{l}\text { Lymphocytopenia }\left(<1000 / \mathrm{mm}^{3}\right) \text { : } \\
10 / 10(1000 \%) \%\end{array}$ & CRP: $4.6(3-11.8) \mathrm{mg} / \mathrm{dL}$ & $\operatorname{CRP}(>5 \mathrm{mg} / \mathrm{dL}): 4 / 10(40 \%)$ \\
\hline Cavagna et al. [26] & $\mathrm{NA}$ & $\begin{array}{l}\text { Hospitalized: } 5 \\
\text { Radiological evidence of pneumonia: } \\
5\end{array}$ & $\mathrm{NA}$ & NA & $\mathrm{NA}$ & NA & $\mathrm{NA}$ \\
\hline Fung et al. [27] & $\begin{array}{l}\text { Fever: } 7(100 \%) \\
\text { Cough. } 6(86 \%) \\
\text { Dyspnea: } 3(43 \%)\end{array}$ & $\begin{array}{l}\text { Hospitalized: } 5(72 \%) \\
\text { Radidological evidence of pneumonia: } \\
3(60 \%) \\
\text { Outpatient: } 2(28 \%)\end{array}$ & $\begin{array}{l}\text { ICU: } 2 / 5(40 \%) \\
\text { ARDS: } 2 / 5(40 \%) \\
\text { MV: } 2 / 5(40 \%) \\
\text { AKI: } 35 \text { (460\%) } \\
\text { RRT: } 1 / 3(33 \%)\end{array}$ & $\begin{array}{l}\text { WBC: } 4300(2800-5800) / \mathrm{mm}^{3} \\
\text { Lymphocyte: } 280(80-1480) / \mathrm{mm}^{3}\end{array}$ & $\begin{array}{l}\text { Lymphocytopenia }\left(<1000 / \mathrm{mm}^{3}\right): 6 / 7 \\
\left(86^{\circ} \%\right)\end{array}$ & $\begin{array}{l}\text { CRP: } 4.9(2.3-17.7) \mathrm{mg} / \mathrm{dL} \\
\text { Procalcitonin: } 0.09(0.03-0.31) \mathrm{ng} / \mathrm{mL}\end{array}$ & $\begin{array}{l}\mathrm{CRP}(>5 \mathrm{mg} / \mathrm{dL}): 2 / 5(40 \%) \\
\text { Procalcitonin }(>0.2 \mathrm{ng} / \mathrm{mL}): 1 / 7(14 \%)\end{array}$ \\
\hline Abrishami et al. [28] & $\begin{array}{l}\text { Fever: } 9(75 \%) \\
\text { Dyspnea: } 5(42 \%) \\
\text { Cought } 9(75 \%) \\
\text { GIs: } 3(25 \%)\end{array}$ & $\begin{array}{l}\text { Hospitalized: } 12(100 \%) \\
\text { Radiological evidence of pneumonia: } \\
12 \text { (100\% } \\
\text { Bilateral infiltrates: } 8(67 \%) \\
\text { Unilateral infiltrates } 4(33 \%)\end{array}$ & $\begin{array}{l}\text { ICU: } 10 / 12(83 \%) \\
\text { ARDS: } 912(17 \% \%) \\
\text { MV: } 9 / 12(75 \%) \\
\text { AKI: NA } \\
\text { RRT: NA }\end{array}$ & $\begin{array}{l}\text { WBC: } 5550(2000-12,200) / \mathrm{mm}^{3} \\
\text { Lymphocyte: } 819(9001480) / \mathrm{mm}^{3} \\
\text { Platalelts: } 154 \times 10^{3}\left(54 \times 10^{3}-485 \times\right. \\
\left.10^{3}\right)\end{array}$ & $\begin{array}{l}\text { Lymphocytopenia }\left(<1000 / \mathrm{mm}^{3}\right) \text { : } \\
\text { 7/12(58\%) }\end{array}$ & CRP: $3.0(0.3-6.6) \mathrm{mg} / \mathrm{dL}$ & $\mathrm{CRP}(>5 \mathrm{mg} / \mathrm{dL}): 2 / 12(17 \%)$ \\
\hline
\end{tabular}


Table A2. Cont.

\begin{tabular}{|c|c|c|c|c|c|c|c|}
\hline \multirow{2}{*}{ Study } & \multirow{2}{*}{ Clinical Presentation } & \multirow{2}{*}{ Respiratory Disease Severity } & \multirow{2}{*}{$\begin{array}{l}\text { Intensive Care Unit Admission \& } \\
\text { Organ Failure (Respiratory or Renal) }\end{array}$} & \multicolumn{2}{|l|}{ Hematological Data } & \multicolumn{2}{|l|}{ Serum Markers } \\
\hline & & & & Median (Range) & Significant Decline $n(\%)$ & Median (Range) & Significant Change $n(\%)$ \\
\hline Hoek et al. [29] & $\mathrm{NA}$ & $\begin{array}{l}\text { Hospitalized: } 16 \\
\text { Radiological evidence of pneumonia: } \\
16(100 \%)\end{array}$ & $\begin{array}{l}\text { ICU: } 1 / 16(6.2 \%) \\
\text { MV: } 1 / 16(6.2 \%) \\
\text { AKI: NA } \\
\text { RRT: NA }\end{array}$ & $\mathrm{NA}$ & $\mathrm{NA}$ & NA & NA \\
\hline Mella et al. [30] & $\begin{array}{l}\text { Fever: } 6(100 \%) \\
\text { Dyspea: } 2(33 \%) \\
\text { Cough: } 3(50 \%) \\
\text { Gls: } 2(33 \%)\end{array}$ & $\begin{array}{l}\text { Hospitalized: } \\
6(100 \%)\end{array}$ & $\begin{array}{l}\text { ICU: } 4 / 6(67 \%) \\
\text { MV: } 4 / 6(67 \%) \\
\text { AKI: } 2 / 6(33 \%) \\
\text { RRT: } 2 / 6(33 \%)\end{array}$ & $\begin{array}{l}\text { WBC. } 7565(4130-13,660) \\
\text { Lymphocyte: } 385(230-610)\end{array}$ & $\begin{array}{l}\text { Lymphocytopenia }\left(<1000 / \mathrm{mm}^{3}\right): 6 / 6 \\
(100 \%)\end{array}$ & $\begin{array}{l}\text { CRP: } 8.2(3.2-17.4) \mathrm{mg} / \mathrm{dL} \\
\text { Procalcitonin: } 0.29(0.12-24.1) \mathrm{ng} / \mathrm{mL}\end{array}$ & $\begin{array}{l}\mathrm{CRP}(>5 \mathrm{mg} / \mathrm{dL}): 4 / 6(67 \%) \\
\text { Procalcitonin }(>0.2 \mathrm{ng} / \mathrm{mL}): 3 / 6(50 \%)\end{array}$ \\
\hline Montagud-Marrahi et al. [31] & $\mathrm{NA}$ & $\begin{array}{l}\text { Majior: } 26(79 \%)-\text { Hospitalized Minor: } \\
7(21 \%) \text {-utpatientRadiological } \\
\text { evidence of preumonia: } 24(73 \%)\end{array}$ & $\begin{array}{l}\text { ICU: } 13 / 26(50 \%) \\
\text { MV: } 2 / 26(7.7 \%) \\
\text { RRT: NA }\end{array}$ & $\mathrm{NA}$ & $\mathrm{NA}$ & NA & $\mathrm{NA}$ \\
\hline Malberti et al. [33] & $\mathrm{NA}$ & $\begin{array}{l}\text { Hospitalized: } \\
11(100 \%)\end{array}$ & $\begin{array}{l}\text { ICU: } 3 / 11 \\
\text { NIV: } 4 / 11(36 \%) \\
(27 \%) \\
\text { MV: } 3 / 11(27 \%) \\
\text { AKII } 5 / 111(45 \%) \\
\text { RRT: } 2 / 5(40 \%)\end{array}$ & NA & NA & NA & $\mathrm{NA}$ \\
\hline Lubetzky et al. [34] & $\begin{array}{l}\text { Fever: } 30(77 \%) \\
\text { Dyspnea. } 21(54 \%) \text { Cough: } 22(56 \%) \\
\text { Gls: } 19(48 \%)\end{array}$ & $\begin{array}{l}\text { Hospitalized: } 39(100 \%) \\
\text { Radiological evidence of pneumonia: } \\
\left.32 / 37\left(866^{\circ}\right)\right)\end{array}$ & $\begin{array}{l}\text { ICU: } 12 / 39(31 \%) \\
\text { MV: } 9 / 39(32 \%) \\
\text { AKI: } 20 / 39(51 \%) \\
\text { RRT: } 5 / 20(25 \%)\end{array}$ & $\begin{array}{l}\text { WBC: NA (2500-9900) } \\
\text { Platelets: NA: } \\
\text { Lymphocyte: } 600 \text { (300-1000) }\end{array}$ & $\mathrm{NA}$ & 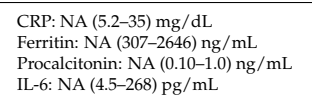 & NA \\
\hline Crespo et al. [36] & $\begin{array}{l}\text { Fever: } 340(82 \%) \\
\text { Dyspnea: } 181(43.7 \%) \\
\text { Cough: } 62(70 \%) \\
\text { GIs: } 152(36.7 \%)\end{array}$ & $\begin{array}{l}\text { Hospitalized: } 380(92 \%) \\
\text { Radiological evidence of pneumonia: } \\
337(89 \%)\end{array}$ & $\begin{array}{l}\text { ICU: } 50 / 380(13 \%) \\
\text { MV: } 73 / 380(19 \%)\end{array}$ & $\mathrm{NA}$ & $\mathrm{NA}$ & $\mathrm{NA}$ & $\mathrm{NA}$ \\
\hline Fava et al. [37] & $\begin{array}{l}\text { Fever: } 72(81 \%) \\
\text { Dyspnea: } 34(38 \%) \\
\text { Cough: } 62(70 \%) \\
\text { GIs: } 27(30 \%)\end{array}$ & $\begin{array}{l}\text { Hospitalized: } 89(100 \%) \text { Radiological } \\
\text { evidence of pnumounona: } 67 / 89(75 \%)\end{array}$ & $\begin{array}{l}\text { ICU: 17/89 (19\%) } \\
\text { ARDS: } 47 / 89(53 \%) \\
\text { MV: } 8 / 89(9 \%)\end{array}$ & $\mathrm{NA}$ & $\begin{array}{l}\text { Lymphocytopenia }\left(<1000 / \mathrm{mm}^{3}\right) \text { : } \\
37 / 89(17 \%)\end{array}$ & NA & $\mathrm{NA}$ \\
\hline Zhang et al. [38] & $\begin{array}{l}\text { Fever: } 5(100 \%) \\
\text { Dyspnea: } 0(0 \%) \\
\text { Cough: }(100 \%) \\
\text { Gls: } 0(0 \%)\end{array}$ & $\begin{array}{l}\text { Hospitalized: } 5(100 \%) \\
\text { Radiological evidence of pneumonia: } \\
5 / 5(100 \%)\end{array}$ & $\begin{array}{l}\text { ICU: } 0 / 5(0 \%) \\
\text { ARD: NA } \\
\text { MV: } 0 / 5(0 \%) \\
\text { AKI: } 1 / 5(20 \%) \\
\text { RRT: } 0 / 1(0 \%)\end{array}$ & $\begin{array}{l}\text { WBC } 6.42 \pm 1.09 / \mathrm{mm}^{3} \\
\text { Platelets: } 186.040 .0 / \mathrm{mm}^{3} \\
\text { Lymphocyte: } 0.58 \pm 0.22 / \mathrm{mm}^{3}\end{array}$ & $\begin{array}{l}\text { Lymphocytopenia }\left(<1000 / \mathrm{mm}^{3}\right): 5 / 5 \\
(100 \%)\end{array}$ & $\mathrm{NA}$ & $\mathrm{NA}$ \\
\hline Cravedi et al. [39] & $\begin{array}{l}\text { Fever: } 96(67 \%) \\
\text { Dyspea: } 97(68 \%) \\
\text { GIs: } 55(38 \%)\end{array}$ & $\begin{array}{l}\text { Hospitalized: } 144(100 \%) \\
\text { Radidiological evidence of pneumonia: } \\
\text { NA }\end{array}$ & $\begin{array}{l}\text { ICU: NA } \\
\text { ARSS: NA } \\
\text { MV: } 42 / 144(29 \%) \\
\text { AI: }: 44 / 144(51 \%) \\
\text { RRT: NA }\end{array}$ & $\begin{array}{l}\text { WBC : } 7.70 \pm 5.65 / \mathrm{mm}^{3} \\
\text { Platelets: } 181.5 \pm 29.0 / \mathrm{mm}^{3} \\
\text { Lymphocyte: } 1.36 \pm 0.78 / \mathrm{mm}^{3}\end{array}$ & $\mathrm{NA}$ & $\begin{array}{l}\text { CRP: } 4.1(1.1-12.5) \mathrm{mg} / \mathrm{dL} \\
\text { Ferritin: } 69919(\mathrm{NA}) \mathrm{ng} / \mathrm{mL} \\
\text { Procalitonin: NA } \\
\text { D-dimer: } 1.97(1.91-2.03) \mu \mathrm{gg} / \mathrm{mL}\end{array}$ & $\mathrm{NA}$ \\
\hline Maritati et al. [40] & $\begin{array}{l}\text { Fever: } 4(80 \%) \\
\text { Dyspnea: } 4(80 \%) \\
\text { Cough: } 4(80 \%)\end{array}$ & $\begin{array}{l}\text { Hospitalized: } 5(100 \%) \\
\text { Radiological evidence of pneumonia: } \\
5(100 \%)\end{array}$ & $\begin{array}{l}\text { ICU: } 3 / 5(60 \%) \\
\text { ARDS: } 45(58 \%) \\
\text { MV: } 5 / 35(60 \%) \\
\text { AKI: } 35 / 5(60 \%) \\
\text { RRT: } 1 / 3(33 \%)\end{array}$ & $\mathrm{NA}$ & $\mathrm{NA}$ & $\mathrm{NA}$ & $\mathrm{NA}$ \\
\hline Elias et al. [41] & $\begin{array}{l}\text { Fever: } 51(77 \%) \\
\text { Dysprea: } 26(39 \%) \\
\text { Cough } 38(58 \%) \\
\text { GIs: } 17(26 \%)\end{array}$ & $\begin{array}{l}\text { Hospitalized: } 60(91 \%) \\
\text { Radiological evidence of pneumonia: } \\
41 / 56(73.2 \%)\end{array}$ & 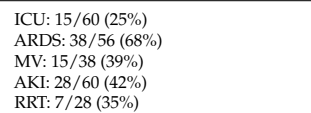 & $\begin{array}{l}\text { WBC: } 6.90 \pm 3.64 / \mathrm{mm}^{3} \\
\text { Lymphocyte: } 0.60 \pm 0.35 / \mathrm{mm}^{3}\end{array}$ & $\mathrm{NA}$ & CRP: $11.5(0.3-33.5) \mathrm{mg} / \mathrm{dL}$ & $\mathrm{NA}$ \\
\hline
\end{tabular}


Table A2. Cont.

\begin{tabular}{|c|c|c|c|c|c|c|c|}
\hline \multirow{2}{*}{ Study } & \multirow{2}{*}{ Clinical Presentation } & \multirow{2}{*}{ Respiratory Disease Severity } & \multirow{2}{*}{$\begin{array}{l}\text { Intensive Care Unit Admission \& } \\
\text { Organ Failure (Respiratory or Renal) }\end{array}$} & \multicolumn{2}{|l|}{ Hematological Data } & \multicolumn{2}{|l|}{ Serum Markers } \\
\hline & & & & Median (Range) & Significant Decline $n(\%)$ & Median (Range) & Significant Change $n(\%)$ \\
\hline Chen et al. [42] & $\begin{array}{l}\text { Fever: } 22(73 \%) \\
\text { Dyspnea: } 20(6 \% \%) \\
\text { Cough: } 22(72 \%) \\
\text { GIs: } 13(43 \%)\end{array}$ & $\begin{array}{l}\text { Hospitalized: } 30(100 \%) \\
\text { Radiological evidence of pneumonia: } \\
\text { NA }\end{array}$ & $\begin{array}{l}\text { ICU: NA } \\
\text { ARDS: NA } \\
\text { MV: } 73(30(23 \%) \\
\text { AKI: } 23 / 30(77 \%) \\
\text { RRT: } 4 / 23(17 \%)\end{array}$ & $\begin{array}{l}\text { WBC: } 6.75 \pm 1.28 / \mathrm{mm}^{3} \\
\text { Lymphocyte: } 0.73 \pm 0.25 / \mathrm{mm}^{3}\end{array}$ & $\begin{array}{l}\text { Lymphocytopenia }\left(<1000 / \mathrm{mm}^{3}\right): \\
30 / 30(100 \%)\end{array}$ & $\begin{array}{l}\text { CRP: } 7.6(4.4-14.7) \mathrm{mg} / \mathrm{dL} \\
\text { Ferritin: } 979(422-1977) \mathrm{ng} / \mathrm{mL} \\
\text { Procalcitonin: } 0.2(0.1-1.3) \mathrm{ng} / \mathrm{mL}\end{array}$ & $\mathrm{NA}$ \\
\hline Mehta et al. [43] & $\begin{array}{l}\text { Fever: } 19(56 \%) \\
\text { Dyspnea: } 18(53 \%) \\
\text { GIs: } 5(15 \%)\end{array}$ & $\begin{array}{l}\text { Hospitalized: } 34 \text { (100\%)Radiological } \\
\text { evidence of pneumonia: } 32 / 34 \text { (94.11) }\end{array}$ & $\begin{array}{l}\text { ICU: NAARDS: NAMV: 13/34(38\%) } \\
\text { AKI: } 18 / 34(53 \%) \\
\text { RRT: } 0 / 18(0 \%)\end{array}$ & Lymphocyte: $0.43 \pm 0.22 / \mathrm{mm}^{3}$ & $\begin{array}{l}\text { Lymphocytopenia }\left(<1000 / \mathrm{mm}^{3}\right) \text { : } \\
32 / 34(94 \%)\end{array}$ & $\mathrm{NA}$ & $\mathrm{NA}$ \\
\hline Silva et al. [44] & $\begin{array}{l}\text { Fever: } 5(100 \%) \\
\text { Dyspnea: } 1(20 \%) \\
\text { Cough: } 2(40 \%) \\
\text { GIs: } 3(60 \%)\end{array}$ & $\begin{array}{l}\text { Hospitalized: } 5(100 \%) \\
\text { Radiological evidence of pneumonia: } \\
4 / 5(80 \%)\end{array}$ & $\begin{array}{l}\text { ICU: } 1 / 5(20 \%) \\
\text { ARDS: } 35(50 \%) \\
\text { AKV: } 15(20 \%) \\
\text { AKI: } 3 / 5(60 \%) \\
\text { RRT: } 0 / 3(0 \%) \\
\end{array}$ & $\begin{array}{l}\text { WBC: } 5.57 \pm 1.77 / \mathrm{mm}^{3} \\
\text { Lymphocyte: } 1.87 \pm 1.10 / \mathrm{mm}^{3}\end{array}$ & $\begin{array}{l}\text { Lymphocytopenia }\left(<1000 / \mathrm{mm}^{3}\right): 1 / 5 \\
(20 \%)\end{array}$ & CRP: $5.6(0.6-12.4)$ mg/dL & $\operatorname{CRP}(>5 \mathrm{mg} / \mathrm{dL}): 2 / 5(40 \%)$ \\
\hline Chavarot et al. [45] & $\begin{array}{l}\text { Fever: } 67 / 93(72 \%) \\
\text { Dyspnea: } 41 / 89(46 \%) \text { Cough: } 55 / 87 \\
\text { (66\%) } \\
\text { GIs: } 32 / 89(36 \%)\end{array}$ & $\begin{array}{l}\text { Hospitalized: } 100(100 \%) \\
\text { Radiological evidence of pneumonia: } \\
40 / 88(48 \% \text { ) }\end{array}$ & $\begin{array}{l}\text { ICU: } 34(34 \%) \\
\text { MV: } 29(29 \%)\end{array}$ & $\mathrm{NA}$ & $\mathrm{NA}$ & $\mathrm{NA}$ & $\mathrm{NA}$ \\
\hline Meester et al. [46] & $\mathrm{NA}$ & $\mathrm{NA}$ & $\mathrm{NA}$ & $\mathrm{NA}$ & NA & $\mathrm{NA}$ & $\mathrm{NA}$ \\
\hline Abolghasemi et al. [47] & $\begin{array}{l}\text { Fever: } 15(62.5 \%) \\
\text { Dyspnea: } 17(71 \%) \\
\text { Cough: } 11(46 \%) \\
\text { GIs: } 6(25 \%)\end{array}$ & $\begin{array}{l}\text { Hospitalized: } 24(100 \%) \\
\text { Radiological evidence of pneumonia: } \\
23 / 24(96 \%)\end{array}$ & ICU: $12 / 24(50 \%)$ & $\mathrm{NA}$ & $\begin{array}{l}\text { Lymphocytopenia }\left(<1100 / \mathrm{mm}^{3}\right): \\
19 / 24(79 \%)\end{array}$ & $\mathrm{NA}$ & $\operatorname{CRP}(>10 \mathrm{mg} / \mathrm{dL}): 24 / 24(100 \%)$ \\
\hline Cucchiari et al. [48] & $\begin{array}{l}\text { Fever: } 26(93 \%) \\
\text { Dyspnea: } 9(32 \%) \\
\text { Cough: } 18 \text { (64\%) } \\
\text { GIs: } 7(25 \%)\end{array}$ & Hospitalized: $28(100 \%)$ & $\begin{array}{l}\text { ICU: } 8 / 28(29 \%) \\
\text { ARDS: } 25 / 28(89 \%) \\
\text { AKII: } 19 / 28(68 \%) \\
\text { RRT: } 3 / 19(0 \%)\end{array}$ & $\mathrm{NA}$ & $\mathrm{NA}$ & $\mathrm{NA}$ & $\mathrm{NA}$ \\
\hline Kute et al. [49] & $\begin{array}{l}\text { Fever: } 220(88 \%) \\
\text { Dyspnea: } 55(22 \%) \\
\text { Cough: } 180(72 \%) \\
\text { GIs: } 60(24 \%)\end{array}$ & $\begin{array}{l}\text { Hospitalized: } 200(80 \%) \\
\text { Radiological evidence of pneumonia: } \\
120 / 250(48 \%)\end{array}$ & $\begin{array}{l}\text { ICU: } 53 / 200(26 \%) \\
\text { MV: } 30 / 200(15 \% \%) \\
\text { AKII 1121/200(60\%) } \\
\text { RRT: } 24 / 121(20 \%)\end{array}$ & $\mathrm{NA}$ & $\mathrm{NA}$ & $\mathrm{NA}$ & $\mathrm{NA}$ \\
\hline Caillard et al. [50] & $\begin{array}{l}\text { Fever: } 180(74 \%) \\
\text { Dyspnea: } 98(40 \%) \\
\text { Cough: } 145(60 \%) \\
\text { GIs: } 97(25 \%)\end{array}$ & Hospitalized: $243(100 \%)$ & $\begin{array}{l}\text { ICU: } 88 / 243(36 \%) \\
\text { MV: } 72(30 \%) \\
\text { AKI: } 106 / 243(43.6 \%) \\
\text { RRT: } 27 / 106(25 \%) \\
\end{array}$ & Lymphocyte: $1.87 \pm 1.10 / \mathrm{mm}^{3}$ & $\mathrm{NA}$ & $\begin{array}{l}\text { CRP: } 6.2(2.7-11.4) \mathrm{mg} / \mathrm{dL} \\
\text { Procalcitonin: } 0.2(0.1-0.48) \mathrm{ng} / \mathrm{mL}\end{array}$ & $\mathrm{NA}$ \\
\hline Kutzler et al. [51] & $\begin{array}{l}\text { Fever: } 5(50 \%) \\
\text { Dyspnea: } 5(50 \%) \\
\text { Coughh } 6(60 \%) \\
\text { GIs: } 2(20 \%)\end{array}$ & Hospitalized: $10(100 \%)$ & $\mathrm{NA}$ & $\mathrm{NA}$ & $\mathrm{NA}$ & $\mathrm{NA}$ & $\mathrm{NA}$ \\
\hline Mairons et al. [52] & $\mathrm{NA}$ & $\mathrm{NA}$ & $\mathrm{NA}$ & $\mathrm{NA}$ & $\mathrm{NA}$ & $\mathrm{NA}$ & $\mathrm{NA}$ \\
\hline Sharma et al. [53] & $\mathrm{NA}$ & NA & $\mathrm{NA}$ & $\mathrm{NA}$ & $\mathrm{NA}$ & NA & $\mathrm{NA}$ \\
\hline Mohamed et al. [54] & $\begin{array}{l}\text { Fever: } 13 / 25(52 \%) \\
\text { Dyspnea: } 17 / 25(68 \%) \\
\text { Cough: } 13 / 24(45 \%) \\
\text { GIs: } 7 / 25(28 \%) \\
\end{array}$ & $\begin{array}{l}\text { Hospitalized: } 27 / 28(96 \%) \\
\text { Radiological evidence of pneumonia: } \\
19 / 28(76 \%)\end{array}$ & $\begin{array}{l}\text { ICU: } 5 / 28(18 \%) \\
\text { AKR: } 14 / 25(56 \%) \\
\text { RRT: } 2 / 14(14.3 \%)\end{array}$ & $\begin{array}{l}\text { WBC: } 8.65 \pm 4.34 / \mathrm{mm}^{3} \\
\text { Lymphocyte: } 0.77 \pm 0.47 / \mathrm{mm}^{3}\end{array}$ & $\mathrm{NA}$ & $\begin{array}{l}\text { CRP: } 9.4(0.7-44.6) \mathrm{mg} / \mathrm{dL} \\
\text { Ferritin: } 1101 \text { (49-5959) ng/mL }\end{array}$ & $\mathrm{NA}$ \\
\hline Felldin et al. [55] & $\mathrm{NA}$ & Hospitalized: $22 / 34(65 \%)$ & $\begin{array}{l}\text { ICU: } 7 / 22(36 \%) \\
\text { MV: } 6 / 22(27 \%) \\
\text { AKI: 14/22(64\%) } \\
\text { RRT: } 5 / 14(36 \%)\end{array}$ & $\mathrm{NA}$ & $\mathrm{NA}$ & $\mathrm{NA}$ & $\mathrm{NA}$ \\
\hline Kates et al. [56] & $\mathrm{NA}$ & $\mathrm{NA}$ & $\mathrm{NA}$ & $\mathrm{NA}$ & $\mathrm{NA}$ & NA & $\mathrm{NA}$ \\
\hline
\end{tabular}


Table A2. Cont.

\begin{tabular}{|c|c|c|c|c|c|c|c|}
\hline \multirow{2}{*}{ Study } & \multirow{2}{*}{ Clinical Presentation } & \multirow{2}{*}{ Respiratory Disease Severity } & \multirow{2}{*}{$\begin{array}{l}\text { Intensive Care Unit Admission \& } \\
\text { Organ Failure (Respiratory or Renal) }\end{array}$} & \multicolumn{2}{|l|}{ Hematological Data } & \multicolumn{2}{|l|}{ Serum Markers } \\
\hline & & & & Median (Range) & Significant Decline $n(\%)$ & Median (Range) & Significant Change $\boldsymbol{n}(\%)$ \\
\hline Benotmane et al. [57] & $\begin{array}{l}\text { Fever: } 38 / 40(95 \%) \\
\text { Dyspnea: } 28 / 40(70 \%) \\
\text { Cough: } 11 / 40(77.7 \%) \\
\text { GIs: } 31 / 40(77.5 \%)\end{array}$ & Hospitalized: $40 / 40(100 \%)$ & $\mathrm{NA}$ & $\mathrm{NA}$ & $\mathrm{NA}$ & $\mathrm{NA}$ & $\mathrm{NA}$ \\
\hline Chaudhry et al. [58] & $\mathrm{NA}$ & Hospitalized: $26 / 40(68 \%)$ & $\begin{array}{l}\text { ICU: } 12 / 26(46.1 \%) \\
\text { ARDS: } 11 / 26(42.3 \%) \\
\text { MV: } 11 / 26(42.23 \%) \\
\text { AKI: } 5 / 26(19 \%)\end{array}$ & $\mathrm{NA}$ & $\mathrm{NA}$ & $\mathrm{NA}$ & $\mathrm{NA}$ \\
\hline Craig-Schapiro et al. [59] & $\begin{array}{l}\text { Fever: 53/75 (71\%) } \\
\text { Dyspnea: } 36 / 74(49 \%) \\
\text { Cough. } 44 / 74(59 \%) \\
\text { GIs: } 25 / 73(34 \%)\end{array}$ & $\begin{array}{l}\text { Hospitalized: } 52 / 80(66 \%) \\
\text { Radiological evidence of pneumonia: } \\
39 / 45(87 \%)\end{array}$ & 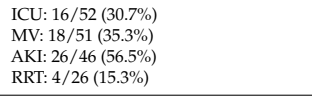 & $\begin{array}{l}\text { WBC: } 5.70 \pm 0.85 / \mathrm{mm}^{3} \\
\text { Lymphocyte: } 0.60 \pm 0.17 / \mathrm{mm}^{3}\end{array}$ & $\mathrm{NA}$ & $\begin{array}{l}\text { CRP: } 12.3(7.2-30.2) \mathrm{mg} / \mathrm{dL} \\
\text { Ferritin: } 1424(503-2428) \mathrm{ng} / \mathrm{mL} \\
\text { Procalcitonin: } 0.3(0.1-10.6) \mathrm{ng} / \mathrm{mL}\end{array}$ & $\mathrm{NA}$ \\
\hline Demir et al. [60] & $\begin{array}{l}\text { Fever: } 25 / 40(62.5 \%) \\
\text { Dyspnea: } 21 / 40(52.5 \%) \\
\text { Cough: } 30 / 40(75 \%) \\
\text { GIs: } 10 / 25(40 \%)\end{array}$ & Hospitalized: 40/40 (100\%) & $\begin{array}{l}\text { ICU: } 7 / 40(18 \%) \\
\text { MV: } 6 / 40(15 \%)\end{array}$ & $\begin{array}{l}\text { WBC: } 5.15 \pm 0.75 / \mathrm{mm}^{3} \\
\text { Lymphocyte: } 0.72 \pm 0.14 / \mathrm{mm}^{3}\end{array}$ & $\mathrm{NA}$ & $\begin{array}{l}\text { CRP: } 4.5(2.4-8.8) \mathrm{mg} / \mathrm{dL} \\
\text { Ferritin: } 358 \text { (173-1374) } \mathrm{ng} / \mathrm{mL} \\
\text { Procalcitonin } 0.2 .2(0.1-1.3) \text { ng } / \mathrm{mL} \\
\text { D-dimer: } 0.36(0.17-0.99) \mu \mathrm{g} / \mathrm{mL}\end{array}$ & $\mathrm{NA}$ \\
\hline Hilbrands et al. [61] & $\begin{array}{l}\text { Fever: } 155 / 271(57 \%) \\
\text { Dyspnea: } 107 / 271(39 \%) \\
\text { Cough: } 136 / 271(50 \%) \\
\text { GIs: } 65 / 271(24 \%) \\
\end{array}$ & Hospitalized: 271/305 (89\%) & ICU: $58 / 271(40 \%)$ & $\mathrm{NA}$ & $\mathrm{NA}$ & $\mathrm{NA}$ & $\mathrm{NA}$ \\
\hline Pascual et al. [62] & $\begin{array}{l}\text { Fever: } 15 / 24(63 \%) \\
\text { Dyspnea: } 14 / 24(58 \%) \\
\text { Cough: } 14 / 24(58 \%) \\
\text { GIs: } 3 / 24(13 \%)\end{array}$ & Hospitalized: $24 / 24(100 \%)$ & $\begin{array}{l}\text { ICU: } 4 / 24(17 \%) \\
\text { MVI } 9 / 24(28 \%) \\
\text { AKI: } 13 / 24(54 \%)\end{array}$ & $\mathrm{NA}$ & $\begin{array}{l}\text { Lymphocytopenia }\left(<1100 / \mathrm{mm}^{3}\right) \text { : } \\
24 / 24(100 \%)\end{array}$ & $\mathrm{NA}$ & $\mathrm{NA}$ \\
\hline Rodriguez-Cubillo et al. [63] & $\begin{array}{l}\text { Fever: } 23 / 29(79 \%) \\
\text { Dyspnea: } 12 / 29(41 \%) \\
\text { Cough: } 18 / 29(12 \%) \\
\text { GIs: } 14 / 29(48 \%)\end{array}$ & $\begin{array}{l}\text { Hospitalized: } 29 / 29(100 \%) \\
\text { Radiological evidence of pneumonia: } \\
18 / 29(62 \%)\end{array}$ & $\begin{array}{l}\text { ICU: } 5 / 29(17 \%) \\
\text { MV: } 4 / 29(14 \%) \\
\text { AKI: } 14 / 29(48 \%) \\
\text { RRT: } 3 / 14(21 \%)\end{array}$ & $\mathrm{NA}$ & $\mathrm{NA}$ & $\mathrm{NA}$ & NA \\
\hline Sanchez-Alvarez et al. [64] & $\mathrm{NA}$ & Hospitalized: $437 / 470(93 \%)$ & $\mathrm{NA}$ & $\mathrm{NA}$ & $\mathrm{NA}$ & $\mathrm{NA}$ & $\mathrm{NA}$ \\
\hline Oto et al. [65] & $\begin{array}{l}\text { Fever: 70/109 (64\%) } \\
\text { Dyspnea: } 53 / 109(41 \%) \\
\text { Cough: } 72 / 109(66 \%) \\
\text { GIs: } 12 / 109(11 \%) \\
\end{array}$ & Hospitalized: 109/109 (100\%) & $\begin{array}{l}\text { ICU: } 22 / 109(20 \%) \\
\text { MV: } 19 / 109(17 \%) \\
\text { AKI } 461109(42 \%) \\
\text { RRT: } 4 / 46(9 \%)\end{array}$ & $\mathrm{NA}$ & $\begin{array}{l}\text { Lymphocytopenia }\left(<1100 / \mathrm{mm}^{3}\right) \text { : } \\
77 / 108(71 \%)\end{array}$ & $\mathrm{NA}$ & $\operatorname{CRP}(>5 \mathrm{mg} / \mathrm{dL}): 47 / 109(43 \%)$ \\
\hline Ellhadedy et al. [66] & $\begin{array}{l}\text { Fever: } 5 / 8(63 \%)) \\
\text { Dyspnea: } 2 / 8(25 \%) \\
\text { Cough: } 5 / 8(633 \%)\end{array}$ & $\begin{array}{l}\text { Hospitalized: } 8 / 8(100 \%) \\
\text { Radiological evidence of pneumonia: } \\
5 / 8(63 \%)\end{array}$ & $\begin{array}{l}\text { ICU: } 1 / 8(13 \%) \\
\text { MV: } 1 / 8(13 \%) \\
\text { AKI: } 2 / 8(25 \%) \\
\text { RRT: } 1 / 2(50 \%)\end{array}$ & $\mathrm{NA}$ & $\begin{array}{l}\text { Lymphocytopenia }\left(<1100 / \mathrm{mm}^{3}\right): 8 / 8 \\
(100 \%)\end{array}$ & CRP: $12.74 \pm 10.0 \mathrm{mg} / \mathrm{dL}$ & $\operatorname{CRP}(>5 \mathrm{mg} / \mathrm{dL}): 6 / 8(75 \%)$ \\
\hline
\end{tabular}

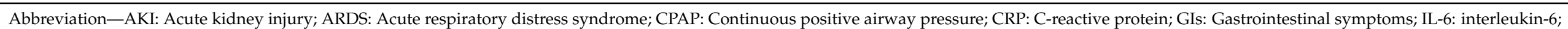
KT: kidney transplant; MV: Mechanical ventilation; NA: Not available/Not applicable; NIV: Non-invasive ventilation; RRT: Renal replacement therapy; WBC: White blood cells. 
Table A3. Reported interventions and outcomes for KT patients with COVID-19.

\begin{tabular}{|c|c|c|c|c|}
\hline Study & Modification of Immunosuppression & Treatments/Therapies & Outcomes & Remarks \\
\hline Akalin et al. [19] & $\begin{array}{ll}\mathbf{0} & \text { Tacrolimus withheld: } 6 / 28(21 \%) \\
\text { MMM withheld: } 24 / 28(88 \%) \\
\text { High-dose steroid: } 2 / 28(7 \%)\end{array}$ & 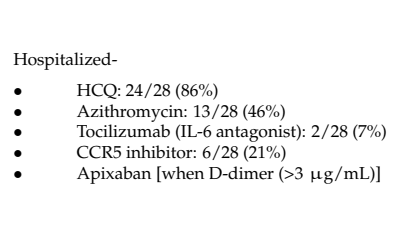 & $\begin{array}{ll}\text { Inpatient death: } 8 / 28(28 \%) \\
\text { Still hospitalized: } 12 / 28(43 \%)\end{array}$ & 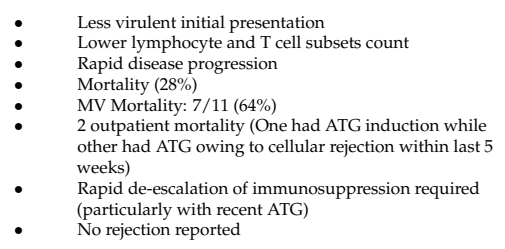 \\
\hline Bossini et al. [20] & $\begin{array}{ll}\text { CNI withheld: NA } \\
\text { MMF witheld: } \mathrm{NA} \\
\text { Increased methyl prednisolone } 16 \mathrm{mg} / \mathrm{d} \text { or equivalent } \\
\text { prednisone till disease resolution: } 18 / 45 \text { (40\%), whilst } \\
16 / 45 \text { were already maintained with originally } \\
\text { moderate high dose }\end{array}$ & 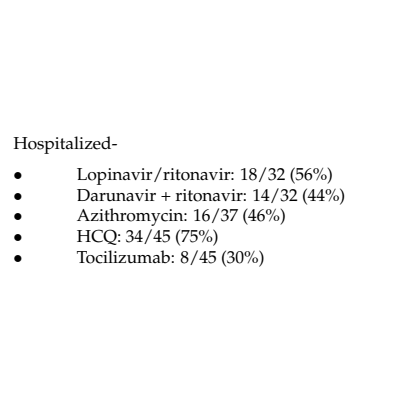 & $\begin{array}{ll}\text { : Inpatient death: } 15 / 45(33 \%) \\
\text { Discharged: } 27 / 45(66 \%)\end{array}$ & 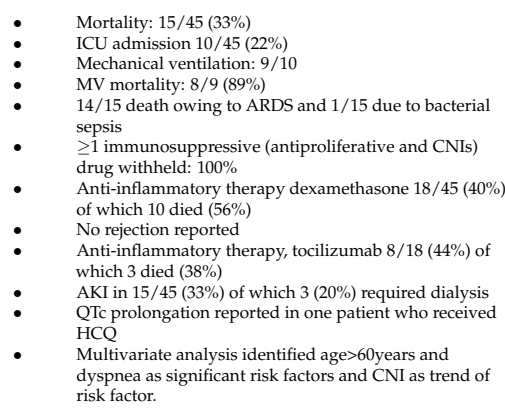 \\
\hline Trujillo et al. [21] & 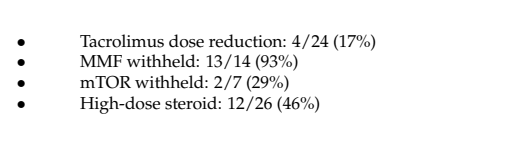 & 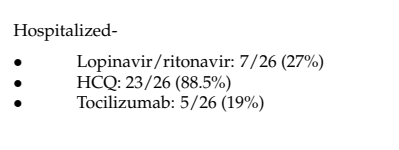 & $\begin{array}{ll}\text { Inpatient death: } 6 / 26(23 \%) \\
\vdots & \text { Still hospitalized: } 13 / 26(50 \%) \\
& \text { Discharged: } 7 / 26(27 \%)\end{array}$ & 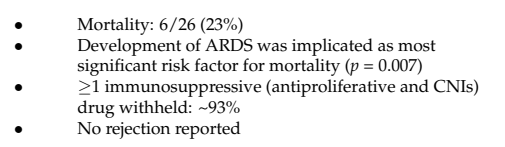 \\
\hline Columbia University KT program [22] & 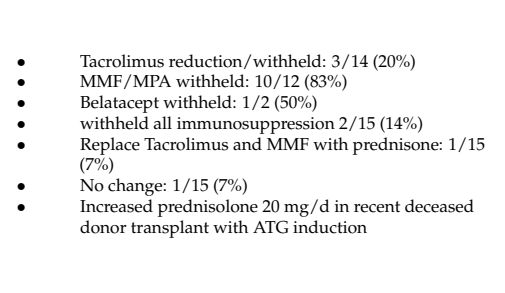 & $\begin{array}{l}\text { Hospitalized- } \\
\vdots \\
\quad \text { HCQ: } 13 / 15(87 \%) \\
\quad \text { Azithromycin: } 9 / 15(60 \%) \\
\quad \text { Tocilizumab: } 1 / 15(7 \%)\end{array}$ & $\begin{array}{ll}\text { Inpatient death: } 2 / 15(13 \%) \\
\vdots & \text { Still hospitatized: } 615(1440 \%) \\
& \text { iischarged: } 8 / 15(535 \%)\end{array}$ & 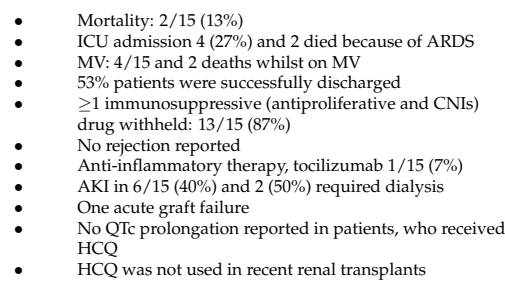 \\
\hline Nair et al. [23] & $\begin{array}{ll}\text { Tacrolimus withheld: } 2 / 9(22 \%) \\
\vdots & \text { MMF MPA witheld: } 8 / 9(89 \%) \\
\text { mTORi: } 1 / 2 \text { (50\%\%) } & \text { (50\%) }\end{array}$ & $\begin{array}{l}\text { Hospitalized- } \\
\vdots \quad \text { HCQ: } 9 / 10(90 \%) \\
\quad \text { Azithromycin: } 9 / 10(90 \%)\end{array}$ & $\begin{array}{ll}\text { : } & \text { Inpatient death: } 3 / 10(30 \%) \\
\text { Discharged: } 7 / 10(70 \%)\end{array}$ & 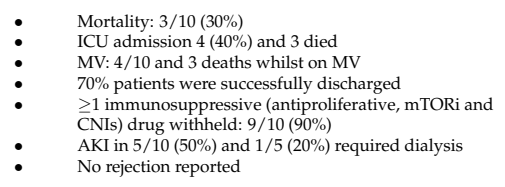 \\
\hline
\end{tabular}




\section{Table A3. Cont.}

\begin{tabular}{|c|c|c|c|c|}
\hline Study & Modification of Immunosuppression & Treatments/Therapies & Outcomes & Remarks \\
\hline Fernandez-Ruiz et al. [24] & 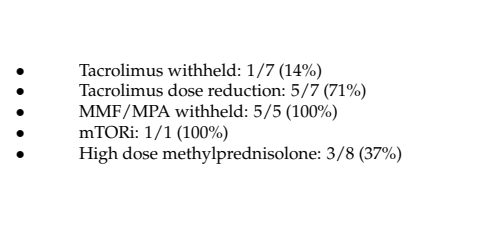 & $\begin{array}{l}\text { Hospitalized- } \\
\quad \begin{array}{l}\text { Lopinavir/ritonavir: } 5 / 8(63 \%) \\
\quad \text { HCI: } 7 / 8(88 \%) \\
\text { IVIg: } 1 / 8(12 \%)\end{array}\end{array}$ & $\begin{array}{ll}: & \text { Total death: } 2 / 8(25 \%) \\
& \text { Discharged: } 1 / 8(12 \%)\end{array}$ & 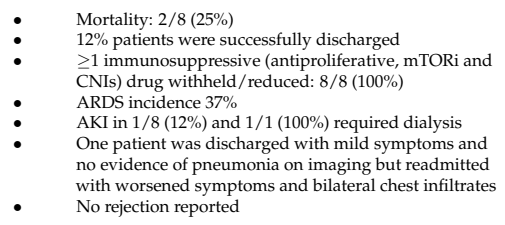 \\
\hline Zhu et al. [25] & 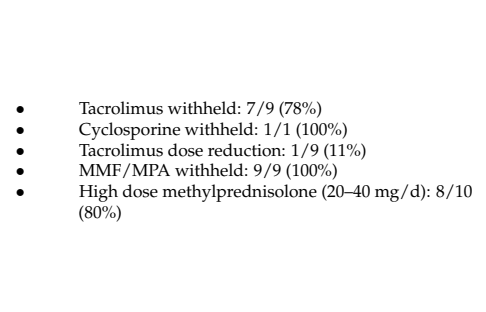 & $\begin{array}{ll}: & \text { Umifenoviri: } 7 / 10(70 \%) \\
& \text { Oseltamivir: } 2 / 10(20 \%) \\
& \text { Ribavarin: } 1 / 110(10 \%) \\
& \text { Ganciclovir: } 1 / 110 \\
\text { IVIg: } 7 / 10(70 \%)\end{array}$ & $\begin{array}{ll}\text { Inpatient death: } 1 / 10(10 \%) \\
\vdots & \text { IDU: } 3 / 10(103 \%)(10 \%) \\
\text { Discharged: } 8 / 10(80 \%)\end{array}$ & 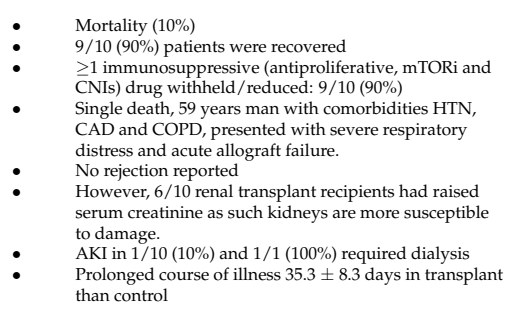 \\
\hline Cavagna et al. [26] & $\mathrm{NA}$ & NA & $\begin{array}{ll} & \text { Inpatient death: } 0 / 5(0 \%) \\
\vdots & \text { Improved: } 3 / 5(60 \%)(6 \%) \\
\text { Stable condition: } 2 / 5(40 \%)\end{array}$ & $\begin{array}{l}\text { Mortality: } 0 / 5(0 \%) \\
\text { Limited information available }\end{array}$ \\
\hline Abrishami et al. [28] & $\begin{array}{ll}\text { Immunosuppressive dosing reduced } \\
\text { Oral steroid changed to intravenous steroid }\end{array}$ & $\begin{array}{ll}\mathbf{0} & \text { Hospitalized- } \\
& \text { Lopinavir//ritonavir: } 12 / 12(100 \%) \\
\text { HCC: } 12212(100 \%) \\
\text { IVIg: if rising creatinine or hypoxemia }\end{array}$ & 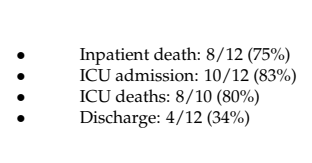 & 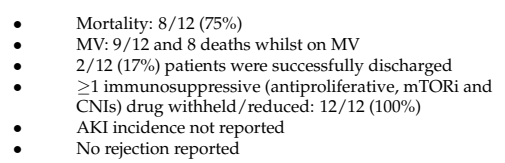 \\
\hline
\end{tabular}




\section{Table A3. Cont.}

\begin{tabular}{|c|c|c|c|c|}
\hline Hoek et al. [29] & $\begin{array}{ll}\text { : } & \text { Explicit details not available } \\
\text { Tacrolinus levels were not modified except in one } \\
\text { patient in whom trough concentration was high } \\
\text { MMF was stopped in severe cases only } \\
\text { M }\end{array}$ & $\begin{array}{l}\text { Hospitalized- } \\
\text { - } \quad \text { Azithromycin to most hospitalized pts }\end{array}$ & $\begin{array}{ll}\text { Inpatient death: } 4 / 1 / 16(25 \%) \\
\text { ICU admission: } 1 / 16(6 \%) \\
\text { ICU deaths: } 1 / 1(100 \%)\end{array}$ & 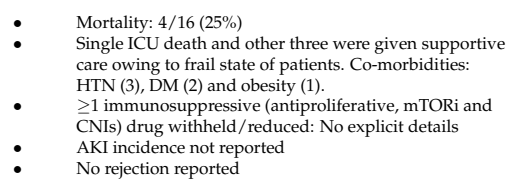 \\
\hline Mella et al. [30] & $\begin{array}{ll}\text { Tacrolimus withheld: } 4 / 6(67 \%) \\
\text { MMF withheld: } 2 / 3(33 \%) \% \\
\text { Increased methylprednisolone } 20-40 \mathrm{mg} / \mathrm{d} \text { ): } 6 / 6\end{array}$ & $\begin{array}{ll}\text { Darunavir + ritonavir: } 2 / 6(33 \%) \\
\text { HCQ: } 2 / 6(33 \%) \\
\text { Tocilizumab: } 6 / 6(100 \%)\end{array}$ & $\begin{array}{ll}\text { Inpatient death: } 4 / 6(67 \%) \\
\vdots & \text { IIU admisision } 4 / 6(66 \%) \\
& \text { ICU deaths: } 4 / 4(100 \%)\end{array}$ & $\begin{array}{ll}: & \text { Mortality } 4 / 6(67 \%) \\
\text { All } 4 \text { IIU death and one had recent KT with } \\
\text { thyogloulininduction } \\
\text { MV: 4/6and } 4 \text { deaths whilst on MV } \\
\quad \geq 1 \text { immunouppressive drug witheld: } 67 \% \\
\quad \text { No rejection observed } \\
\quad \text { No QTe prolongation reported in patients who received } \\
\text { HCQ }\end{array}$ \\
\hline Montagud-Marrahi et al. [31] & $\begin{array}{l}\text { Immunosuppressive dosing reduced } \\
\text { Increase steroid: } 13 / 26(50 \%)\end{array}$ & 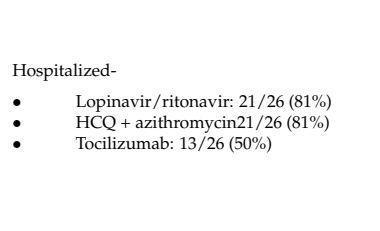 & $\begin{array}{ll}\text { Inpatient death: } 2 / 26(7 \%) \\
\vdots & \text { ICU addiassion: } 13 / 26(50 \%) \\
& \text { ICU deaths: } 2 / 13(14 \%)\end{array}$ & 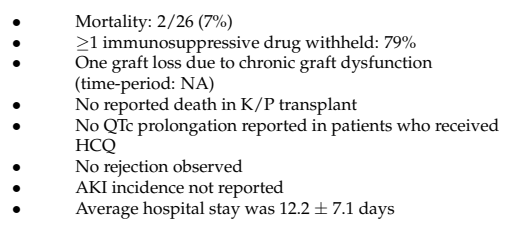 \\
\hline Banerjee et al. [32] & $\begin{array}{ll}: & \text { Tacrolimus withheld/reduced: } 2 / 6(32 \%) \\
\text { MMF withheld: } 5 / 5(100 \%) \\
\text { Azathioprine withheld: } 1 / 2 \\
\text { Increase steroid: } 1 / 5(20 \%)\end{array}$ & NA & $\begin{array}{ll}\text { Inpatient death: } 1 / 5(20 \%) \\
& \text { ICU admission: } 4 / 5(80 \%) \\
& \text { ICU deaths: } 1 / 4(25 \%) \\
& \text { Still admitted: } 3 / 5(60 \%)\end{array}$ & 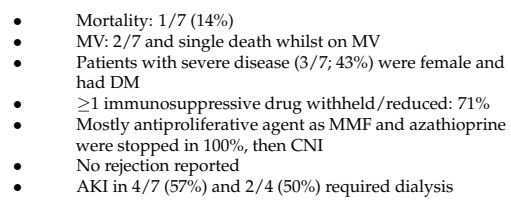 \\
\hline Malberti et al. [33] & $\begin{array}{ll}\text { Tacrolimus withheld: } 8 / 11(73 \%) \\
\text { MMF withheld: 11/11 (100\%) } \\
\text { Increased prednisolone or rrednisone to } 15 \mathrm{mg} / \mathrm{d} \text { or } 25 \\
\text { mg/d) till disease resolution: } 4 / 11 \text { (36\%) }\end{array}$ & 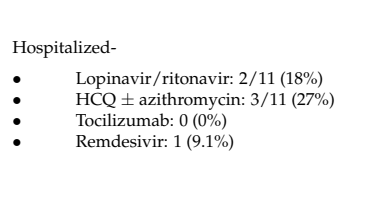 & $\begin{array}{ll}\text { Inpatient death: } 4 / 11(36 \%) \\
& \text { ICU admission: } 3 / 11(27 \%) \\
& \text { ICU deaths } 3 / 3(100 \%) \\
& \text { Discharged: } 7 / 11(64 \%)\end{array}$ & 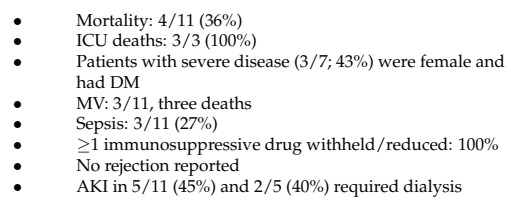 \\
\hline
\end{tabular}




\section{Table A3. Cont.}

\begin{tabular}{|c|c|c|c|c|}
\hline Lubetzky et al. [34] & $\begin{array}{ll}: & \text { Tacrolimus dose reduction: } 20 / 37(54 \%) \\
: & \text { MMF/MPA withheld: } 24 / 38(63 \%) \\
& \text { MMF dose reduction } 101 / 38(26 \%) \\
& \text { Increase steroid: } 5 / 39(13 \%)\end{array}$ & $\begin{array}{l}\text { Hospitalized- } \\
: \quad \text { HCQ: } 31 / 39(80 \%) \\
\quad \quad \text { Tocilizumab: } 2 / 39(5 \%) \\
\quad \text { Remdesivir: } 2 / 39(5 \%)\end{array}$ & 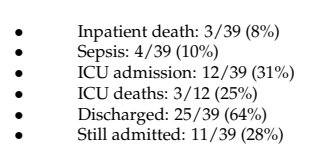 & 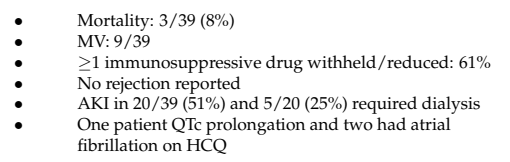 \\
\hline Devresee et al. [35] & 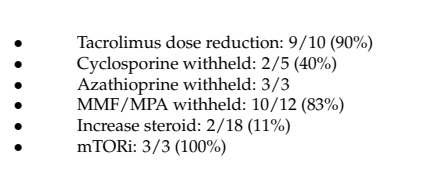 & $\begin{array}{l}\text { Hospitalized- } \\
\text { - } \quad \text { HCQ: } 15 / 18(83 \%)\end{array}$ & 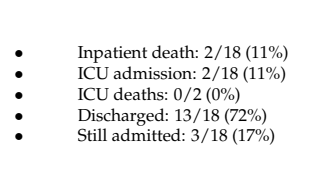 & 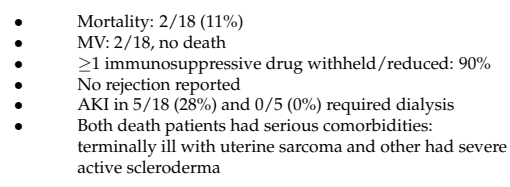 \\
\hline Crespo et al. [36] & Increase steroid: 203/380 (53\%) & 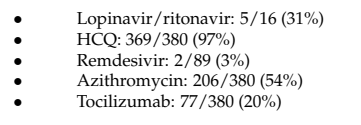 & $\begin{array}{ll}\text { Inpatient death: } 107 / 380(28 \%) \\
\quad & \text { ICU admission: } 50 / 380(13.1 \%) \\
& \text { ICU deaths: } 33 / 50(66 \% \%)\end{array}$ & $\begin{array}{ll}\text { Mortality: } 107 / 380(28 \%) \\
\text { ICU deaths: } 33 / 50(66 \%) \\
\text { MV: } 73 / 380 \text { and } 60 \text { deaths while on MV }\end{array}$ \\
\hline Fava et al. [37] & 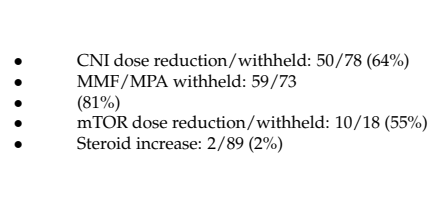 & 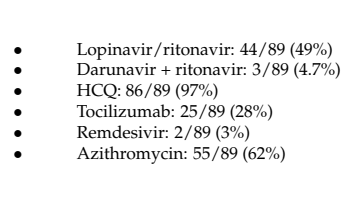 & $\begin{array}{ll}\text { Inpatient death: } 25 / 89(28 \%) \\
\text { ICU admission: } 17 / 89(19 \%) \\
\text { ICU deaths: } 1 / 17(47 \% \%)\end{array}$ & 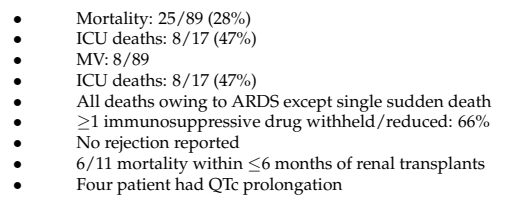 \\
\hline Zhang et al. [38] & $\begin{array}{ll}\text { CNI dose reduction/withheld: } 3 / 5(60 \%) \\
\text { MMF/MPA withheld: } 3 / 5(60 \%) \\
\text { Steroid increase: } 2 / 5(40 \%))\end{array}$ & - $\quad$ Oseltamivir: 5/5 $(100 \%)$ & $\begin{array}{ll}\text { Inpatient death: } 0 / 5(0 \%) \\
\quad & \text { Discharged: } 2 / 5(40 \%) \\
& \text { Still admitted: } 3 / 5(60 \%)\end{array}$ & $\begin{array}{ll}\text { Mortality: } 0 / 5(0 \%) \\
\geq 1 \text { immunosuppressive drug withheld/reduced: } 60 \% \\
\text { Single rejection reported }\end{array}$ \\
\hline Cravedi et al. [39] & $\begin{array}{ll}\text { CNI dose reduction/withheld: } 32 / 131(60 \%) \\
: & \text { MMF MPA withheldd } 91 / 1111(60 \%) \\
\text { Steroid increase: } 95 / 144(66 \%)\end{array}$ & 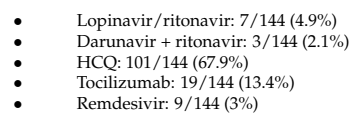 & $\begin{array}{l}\text { Inpatient death: } 46 / 144(32 \%) \\
\quad \text { Discharged: } 98 / 144(68 \%)\end{array}$ & 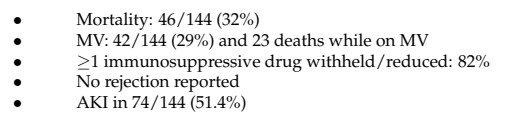 \\
\hline Maritati et al. [40] & $\begin{array}{ll}\text { CNI dose reduction/ withheld: } 5 / 5(100 \%) \\
\text { MMF MPA withheld: } 4 / 4(100 \%) \\
\text { mTORi: } 0 / 1(0 \%) \\
\text { Steroid increase: } 4 / 5(80 \%)\end{array}$ & $\begin{array}{ll}: & \text { Lopinavir/ritonavir: } 1 / 5(20 \%) \\
& \text { HCQ: } 3 / 5(60 \%) \\
\text { Tocilizumab: } 5 / 5(100 \%)\end{array}$ & $\begin{array}{ll}: & \text { Inpatient death: } 2 / 5(40 \%) \\
& \text { Discharged: } 2 / 5(40 \%)\end{array}$ & $\begin{array}{ll}\text { : } & \text { Mortality: } 2 / 5(40 \%) \\
\text { MV: } 3 / 5(60 \% \text { and } 2 \text { deaths while on MV } \\
\geq 1 \text { immunosuppressive drug withheld/reduced: } 100 \% \\
\quad \text { No rejection reported } \\
\text { One patient had long QT so did not receive } \\
\text { One patient was on ECMO and very sick but preserved } \\
\text { graft function } \\
\text { AKI in } 3(60 \%) \text { patients and } 1 \text { had renal replacement } \\
\text { therapy }\end{array}$ \\
\hline
\end{tabular}




\section{Table A3. Cont.}

\begin{tabular}{|c|c|c|c|c|}
\hline Elias et al. [41] & $\begin{array}{l}\text { CNI dose reduction/withheld: } 2 / 57 \text { (4\%) } \\
\text { MMFF/MPA withheld: } 38 / 61(62 \%) \\
\text { Belatacept: } 1 / 6(17 \%)\end{array}$ & $\begin{array}{l}\text { HCQ: } 6 / 60(10 \%) \\
\quad \text { Tocilizumab: } 1 / 60(2 \%)\end{array}$ & $\begin{array}{l}\text { Inpatient death: } 16 / 60(27 \%) \\
\quad \text { Discharged: } 50 / 60(83 \%)\end{array}$ & 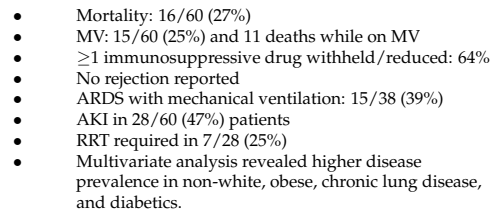 \\
\hline Chen et al. [42] & 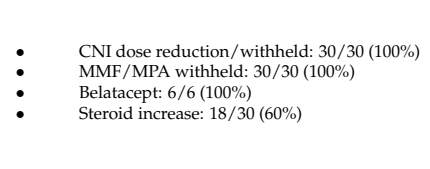 & $\begin{array}{ll}\text { HCQ: } 30 / 30(100 \%) \\
\text { AZi: } 30 / 30(100 \%)\end{array}$ & $\begin{array}{ll}\text { Inpatient death: } 6 / 30(20 \%) \\
\quad & \text { Dischargedd } 23,30(77 \%) \\
& \text { Still admitted: } 1 / 30(3 \%)\end{array}$ & 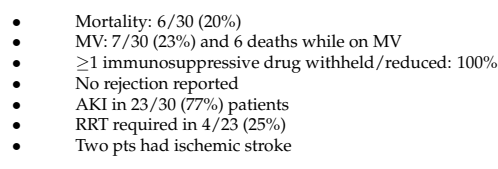 \\
\hline Mehta et al. [43] & $\begin{array}{ll}\text { CNI dose reduction/withheld: } 0 / 30(100 \%) \\
\text { MMF/MPA withheld: } 33 / 34(97 \%)\end{array}$ & $\begin{array}{ll}\mathbf{H} & \text { HCQ: } 33 / 34(97 \%) \\
& \text { Azi: } 27 / 34(79 \%) \\
& \text { Tocilizumab: } 9 / 34(27 \%)\end{array}$ & $\begin{array}{ll}\text { Inpatient death: } 6 / 34(18 \%) \\
\quad & \text { Dischargedd } 26 / 34(76 \%) \\
& \text { Still admitted: } 2 / 34(6 \%)\end{array}$ & 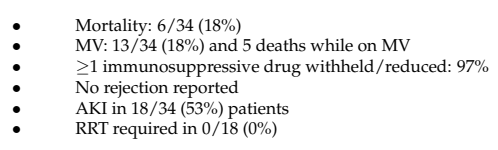 \\
\hline Silva et al. [44] & 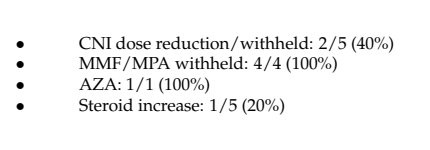 & - $\quad$ HCQ: $3 / 5(60 \%)$ & $\begin{array}{ll}\text { Inpatient death: } 1 / 5(20 \%) \\
\text { Discharged: } 4 / 5(80 \%)\end{array}$ & 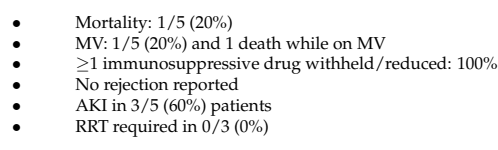 \\
\hline Chavarot et al. [45] & $\begin{array}{ll}\text { : } & \text { CNI dose reduction/withheld: } 36 / 90(40 \%) \\
\text { MMF /MPA withheld: } 56 / 71(79 \%)\end{array}$ & $\begin{array}{ll}\mathbf{0} & \text { HCQ: } 11 / 85(13 \%) \\
& \text { Azi: } 27 / 34(79 \%) \\
\text { Tocilizumab: } 9 / 34(27 \%)\end{array}$ & $\begin{array}{ll}\text { Inpatient death: } 26 / 100(26 \%) \\
\quad & \text { Discharged: } 62 / 100(62 \%) \\
& \text { Still admitted: } 12 / 100(12 \%)\end{array}$ & 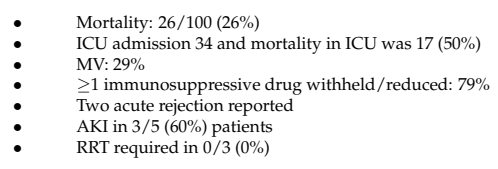 \\
\hline Meester et al. [46] & $\mathrm{NA}$ & $\mathrm{NA}$ & - Inpatient death: $6 / 43(14 \%)$ & Mortality: 6/43(14\%) \\
\hline Abolghasemi et al. [47] & $\mathrm{NA}$ & 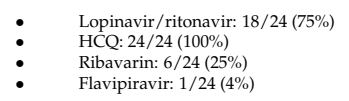 & $\begin{array}{l}\text { Inpatient death: } 10 / 24(42 \%) \\
\quad \text { Discharged: } 14 / 24(58 \%)\end{array}$ & $\begin{array}{l}\text { Mortality 10/24(42\%) } \\
\text { ICU admission 50\% }\end{array}$ \\
\hline
\end{tabular}


Table A3. Cont.

\begin{tabular}{|c|c|c|c|c|}
\hline Cucchiari et al. [48] & - $\quad$ Steroid increase: $8 / 28(29 \%)$ & 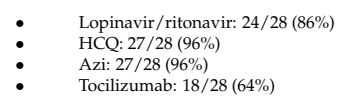 & - Inpatient death: $5 / 28(18 \%)$ & $\begin{array}{l}\text { : } \quad \text { Mortality: } 5 / 28(18 \%) \\
\text { ICU admission } 29 \%\end{array}$ \\
\hline Kute et al. [49] & $\begin{array}{ll}\text { CNI dose reduction/withheld: } 71 / 250(28 \%) \\
\text { MMF MPA witheldd: } 250 / 250(100 \%) \\
\text { Steroid increase: } 100 / 250(40 \%)\end{array}$ & 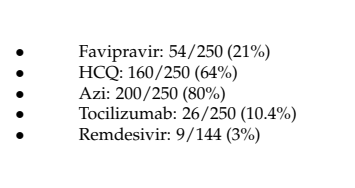 & $\begin{array}{l}\text { Inpatient death: } 29 / 200(18 \%) \\
\text { Discharged: } 156 / 200(78 \%)\end{array}$ & 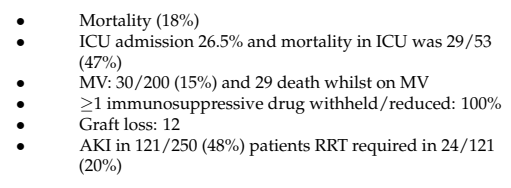 \\
\hline Caillard et al. [50] & 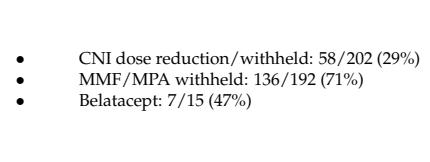 & 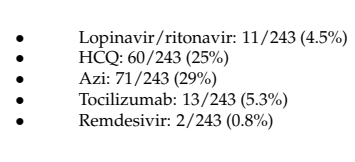 & - Inpatient death: $43 / 243(17.6 \%)$ & 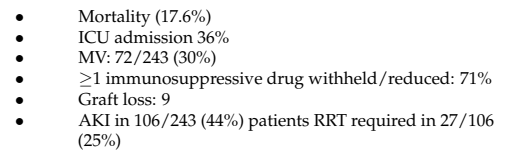 \\
\hline Kutzler et al. [51] & $\begin{array}{ll}: & \text { CNI dose reduction } / \text { withheld: } 2 / 5(40 \%) \\
\vdots & \text { MMF/MPA withheld: } 3 / 5(60 \%) \\
& \text { Belatacept } 1 / 1(100 \%) \\
& \text { Steroid increase: } 3 / 10(30 \%)\end{array}$ & $\begin{array}{ll}\text { HCQ: } 3 / 10(30 \%) \\
: & \text { ALi } 3 / 310(30 \%) \\
& \text { Tocilizumab: } 1 / 110(10 \%)\end{array}$ & - Inpatient death: 6/10 $(60 \%)$ & $\begin{array}{l}\text { Mortality }(60 \%) \\
\geq 1 \text { immunosuppressive drug withheld/reduced: } 60 \%\end{array}$ \\
\hline Mairons et al. [52] & NA & $\mathrm{NA}$ & Inpatient death: $8 / 30(26.6 \%)$ & Mortality (26.6\%) \\
\hline Sharma et al. [53] & $\mathrm{NA}$ & $\mathrm{NA}$ & Inpatient death: $2 / 16(12.5 \%)$ & Mortality $(12.5 \%)$ \\
\hline Mohamed et al. [54] & 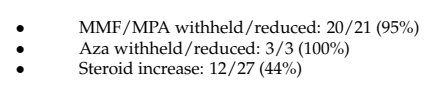 & $\mathrm{NA}$ & Inpatient death: $9 / 28(32 \%)$ & 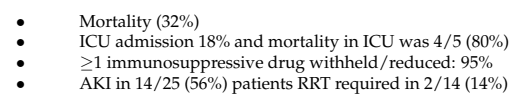 \\
\hline Felldin et al. [55] & $\begin{array}{ll}\text { CNI dose reduction/ withheld: } 9 / 22(41 \%) \\
: & \text { MMF/MP w ithheld: } 12 / 212(100 \%) \\
& \text { Steroid increase: } 3 / 22(14 \%)\end{array}$ & $\mathrm{NA}$ & $\begin{array}{ll}\text { Inpatient death: } 3 / 22(14 \%) \\
\vdots & \text { Discharged: } 16 / 22(72 \%) \\
\text { Still admitted: } 3 / 22(12 \% \%)\end{array}$ & 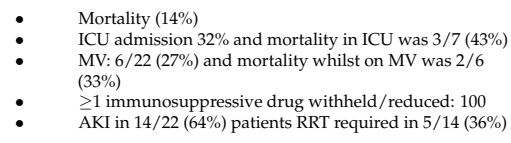 \\
\hline Kates et al. [56] & $\mathrm{NA}$ & $\mathrm{NA}$ & Inpatient death: $57 / 318(18 \%)$ & Mortality $(18 \%)$ \\
\hline Benotmane et al. [57] & $\begin{array}{ll}\text { CNI dose reduction/withheld: } 15 / 35(41 \%) \\
\text { MMF MPA witheldd: } 34 / 34 \text { (100\%) } \\
\text { Steroid increase: } 6 / 40(15 \%)\end{array}$ & $\begin{array}{ll}\text { HCQ: } 15 / 40(37.5 \%) \\
\vdots & \text { Azi: } 26 / 40(65 \%) \\
& \text { Tocilizumab: } 4 / 40(10 \%)\end{array}$ & - Inpatient death: $9 / 40(23 \%)$ & $\begin{array}{ll}\text { : } & \text { Mortality (23\%) } \\
\geq 1 \text { immunosuppressive drug withheld/reduced: } 100\end{array}$ \\
\hline
\end{tabular}


Table A3. Cont.

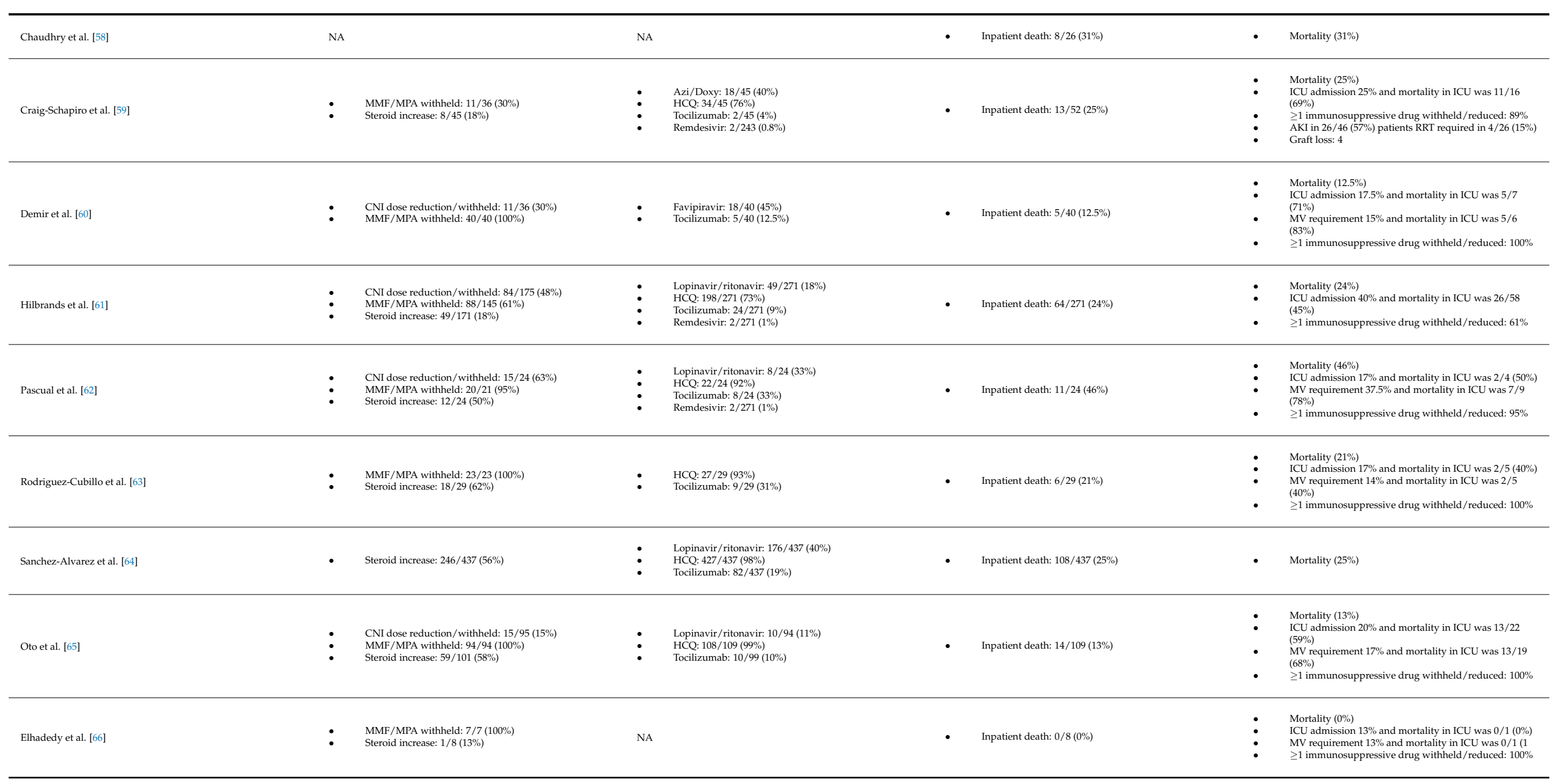

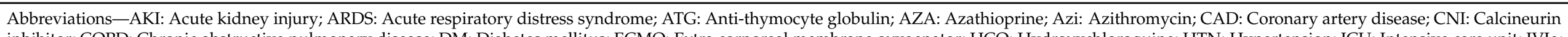

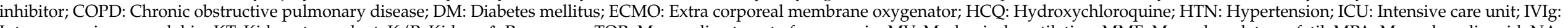

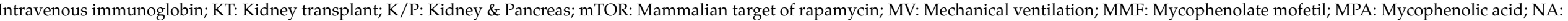

Not available/Not applicable; QTc: Corrected QT interval; RRT: Renal replacement therapy; IL-6 antagonist: Interleukin-6 antagonist (tocilizumab). 
Table A4. Summary statistics outlined as pooled estimates of outcomes of interest.

\begin{tabular}{|c|c|c|c|c|}
\hline \multirow[t]{2}{*}{ Attributes } & \multirow[t]{2}{*}{ Events } & \multirow[t]{2}{*}{ Total } & \multirow[t]{2}{*}{ Studies } & \multirow{2}{*}{$\begin{array}{l}\text { Single Group } \\
\text { Summary }(95 \% \mathrm{CI}) \\
\text { Random-Effects } \\
\text { Model }\end{array}$} \\
\hline & & & & \\
\hline \multicolumn{5}{|c|}{ Demographic variables } \\
\hline Age (years) & NA & 2971 & 44 & $57.08(54.55-59.03)$ \\
\hline Male & 1911 & 2985 & 44 & $0.66(0.61-0.70)$ \\
\hline Caucasians & 401 & 860 & 14 & $0.40(0.21-0.58)$ \\
\hline African-American & 170 & 898 & 15 & $0.27(0.19-0.36)$ \\
\hline Hispanics & 136 & 589 & 13 & $0.19(0.10-0.28)$ \\
\hline $\begin{array}{l}\text { Deceased donor } \\
\text { transplant }\end{array}$ & 492 & 996 & 20 & $0.58(0.40-0.77)$ \\
\hline $\begin{array}{l}\text { Living donor } \\
\text { transplant }\end{array}$ & 497 & 996 & 18 & $0.41(0.21-0.61)$ \\
\hline \multicolumn{5}{|l|}{ Comorbidities } \\
\hline $\mathrm{T} 2 \mathrm{DM}$ & 621 & 1785 & 33 & $0.34(0.29-0.40)$ \\
\hline Hypertension & 1404 & 1733 & 32 & $0.83(0.78-0.88)$ \\
\hline Heart disease & 398 & 1738 & 28 & $0.23(0.17-0.28)$ \\
\hline Chronic lung disease & 233 & 1368 & 19 & $0.15(0.05-0.24)$ \\
\hline Malignancy & 95 & 1010 & 12 & $0.08(0.06-0.11)$ \\
\hline Obesity & 239 & 713 & 14 & $0.36(0.26-0.46)$ \\
\hline Smoking & 155 & 976 & 11 & $0.15(0.10-0.20)$ \\
\hline \multicolumn{5}{|c|}{ Maintenance immunosuppression } \\
\hline $\mathrm{CNI}$ & 1942 & 2307 & 39 & $0.91(0.88-0.94)$ \\
\hline MMF & 1784 & 2325 & 38 & $0.79(0.73-0.85)$ \\
\hline Steroid & 1876 & 2313 & 37 & $0.86(0.82-0.90)$ \\
\hline mTOR inhibitors & 267 & 1934 & 20 & $0.12(0.09-0.16)$ \\
\hline \multicolumn{5}{|c|}{ Presenting parameters and symptoms } \\
\hline Fever & 1716 & 2308 & 37 & $0.77(0.72-0.81)$ \\
\hline Cough & 1362 & 2122 & 35 & $0.65(0.61-0.69)$ \\
\hline Dyspnea & 1003 & 2303 & 37 & $0.48(0.42-0.53)$ \\
\hline $\begin{array}{l}\text { Gastrointestinal } \\
\text { symptoms }\end{array}$ & 711 & 2282 & 34 & $0.29(0.25-0.34)$ \\
\hline \multicolumn{5}{|l|}{ Investigations } \\
\hline Lymphocytopenia & 607 & 802 & 15 & $0.79(0.70-0.89)$ \\
\hline High CRP & 109 & 205 & 9 & $0.60(0.35-0.84)$ \\
\hline $\begin{array}{l}\text { Radiological } \\
\text { evidence of } \\
\text { pneumonia }\end{array}$ & 982 & 1312 & 29 & $0.81(0.74-0.88)$ \\
\hline \multicolumn{5}{|c|}{ Clinical management \& outcome } \\
\hline ARDS & 197 & 344 & 13 & $0.58(0.48-0.69)$ \\
\hline $\begin{array}{l}\mathrm{CNI} \\
\text { withheld/reduced }\end{array}$ & 527 & 1498 & 33 & $0.43(0.30-0.56)$ \\
\hline $\begin{array}{l}\text { MMF } \\
\text { withheld/reduced }\end{array}$ & 1181 & 1393 & 35 & $0.91(0.88-0.94)$ \\
\hline $\begin{array}{l}\text { mTOR inhibitor } \\
\text { withheld/reduced }\end{array}$ & 31 & 47 & 9 & $0.72(0.51-0.94)$ \\
\hline $\begin{array}{l}\text { Increase/pulse } \\
\text { steroid }\end{array}$ & 940 & 2172 & 31 & $0.40(0.30-0.50)$ \\
\hline $\mathrm{HCQ}$ & 1945 & 2623 & 34 & $0.73(0.66-0.80)$ \\
\hline Azithromycin & 739 & 1302 & 17 & $0.62(0.47-0.76)$ \\
\hline Lopinavir/ritonavir & 554 & 1861 & 18 & $0.41(0.29-0.53)$ \\
\hline Tocilizumab & 386 & 2477 & 26 & $0.21(0.16-0.26)$ \\
\hline Remdesivir & 28 & 977 & 8 & $0.03(0.01-0.04)$ \\
\hline ICU admissions & 570 & 2439 & 37 & $0.26(0.22-0.30)$ \\
\hline $\begin{array}{l}\text { Mechanical } \\
\text { ventilation }\end{array}$ & 433 & 1848 & 33 & $0.24(0.20-0.28)$ \\
\hline AKI & 626 & 1260 & 30 & $0.48(0.43-0.53)$ \\
\hline Dialysis & 118 & 534 & 27 & $0.22(0.16-0.29)$ \\
\hline ICU death & 184 & 355 & 27 & $0.53(0.44-0.63)$ \\
\hline $\begin{array}{l}\text { Death on mechanical } \\
\text { ventilator }\end{array}$ & 206 & 285 & 24 & $0.68(0.58-0.79)$ \\
\hline Hospital death & 718 & 3137 & 48 & $0.21(0.19-0.24)$ \\
\hline
\end{tabular}

Abbreviations-AKI: Acute kidney injury: ARDS: Acute respiratory distress syndromes; CNI: Calcineurin inhibitor; CRP: C-reactive protein; T2DM: Diabetes mellitus Type 2; GIs: Gastrointestinal symptoms; HCQ: Hydroxychloroquine: ICU: Intensive care unit; K/P: Kidney and Pancreas; MV: Mechanical ventilation; MMF: Mycophenolate mofetil; MPA: Mycophenolic acid; mTOR: Mammalian target of Rapamycin. † Pooled prevalence was measured as effect size (ES). In the case of age, it is a measure of mean; for the remaining variables, it is expressed as the proportion of individuals (i.e., events) out of a total available sample size based upon inclusion of the index parameters. Single group summary is represented as a pooled effect size (ES) and is presented as a percentage in the Results section. 


\section{References}

1. Wang, L.; He, W.; Yu, X.; Hu, D.; Bao, M.; Liu, H.; Zhou, J.; Jiang, H. Coronavirus Disease 2019 in Elderly Patients: Characteristics and Prognostic Factors Based on 4-Week Follow-Up. J. Infect. 2020, 80, 639-645. [CrossRef]

2. Zheng, K.I.; Gao, F.; Wang, X.-B.; Sun, Q.-F.; Pan, K.-H.; Wang, T.-Y.; Ma, H.-L.; Chen, Y.-P.; Liu, W.-Y.; George, J.; et al. Letter to the Editor: Obesity as a Risk Factor for Greater Severity of COVID-19 in Patients with Metabolic Associated Fatty Liver Disease. Metabolism 2020, 108, 154244. [CrossRef]

3. Zhou, F.; Yu, T.; Du, R.; Fan, G.; Liu, Y.; Liu, Z.; Xiang, J.; Wang, Y.; Song, B.; Gu, X.; et al. Clinical Course and Risk Factors for Mortality of Adult Inpatients with COVID-19 in Wuhan, China: A Retrospective Cohort Study. Lancet 2020, 395, $1054-1062$. [CrossRef]

4. Laupacis, A.; Keown, P.; Pus, N.; Krueger, H.; Ferguson, B.; Wong, C.; Muirhead, N. A Study of the Quality of Life and Cost-Utility of Renal Transplantation. Kidney Int. 1996, 50, 235-242. [CrossRef]

5. Kostro, J.Z.; Hellmann, A.; Kobiela, J.; Skóra, I.; Lichodziejewska-Niemierko, M.; Dębska-Ślizień, A.; Śledziński, Z. Quality of Life After Kidney Transplantation: A Prospective Study. Transplant. Proc. 2016, 48, 50-54. [CrossRef]

6. Kumar, D.; Manuel, O.; Natori, Y.; Egawa, H.; Grossi, P.; Han, S.; Fernández-Ruiz, M.; Humar, A. COVID-19: A Global Transplant Perspective on Successfully Navigating a Pandemic. Am. J. Transplant. 2020, 20, 1773-1779. [CrossRef] [PubMed]

7. Thaunat, O.; Legeai, C.; Anglicheau, D.; Couzi, L.; Blancho, G.; Hazzan, M.; Pastural, M.; Savoye, E.; Bayer, F.; Morelon, E.; et al. IMPact of the COVID-19 Epidemic on the MoRTAlity of Kidney Transplant Recipients and Candidates in a French Nationwide Registry STudy (IMPORTANT). Kidney Int. 2020, 98, 1568-1577. [CrossRef]

8. de Jager, D.J. Cardiovascular and Noncardiovascular Mortality Among Patients Starting Dialysis. JAMA 2009, $302,1782$. [CrossRef]

9. Carrero, J.J.; de Jager, D.J.; Verduijn, M.; Ravani, P.; De Meester, J.; Heaf, J.G.; Finne, P.; Hoitsma, A.J.; Pascual, J.; Jarraya, F.; et al. Cardiovascular and Noncardiovascular Mortality among Men and Women Starting Dialysis. Clin. J. Am. Soc. Nephrol. 2011, 6, 1722-1730. [CrossRef] [PubMed]

10. Boyarsky, B.J.; Werbel, W.A.; Durand, C.M.; Avery, R.K.; Jackson, K.R.; Kernodle, A.B.; Snyder, J.; Hirose, R.; Massie, I.M.; Garonzik-Wang, J.M.; et al. Early National and Center-level Changes to Kidney Transplantation in the United States during the COVID-19 Epidemic. Am. J. Transplant. 2020, 20, 3131-3139. [CrossRef]

11. Stroup, D.F.; Berlin, J.A.; Morton, S.C.; Olkin, I.; Williamson, G.D. MOOSE Guidelines for Meta-Analyses and Systematic Reviews of Observational Studies. JAMA 2000, 283, 2008-2012. [CrossRef]

12. Bonanad, C.; García-Blas, S.; Tarazona-Santabalbina, F.; Sanchis, J.; Bertomeu-González, V.; Fácila, L.; Ariza, A.; Núñez, J.; Cordero, A. The Effect of Age on Mortality in Patients with COVID-19: A Meta-Analysis with 611,583 Subjects. J. Am. Med. Dir. Assoc. 2020, 21, 915-918. [CrossRef] [PubMed]

13. Parohan, M.; Yaghoubi, S.; Seraji, A.; Javanbakht, M.H.; Sarraf, P.; Djalali, M. Risk Factors for Mortality in Patients with Coronavirus Disease 2019 (COVID-19) Infection: A Systematic Review and Meta-Analysis of Observational Studies. Aging Male 2020, 23, 1416-1424. [CrossRef]

14. Wan, X.; Wang, W.; Liu, J.; Tong, T. Estimating the Sample Mean and Standard Deviation from the Sample Size, Median, Range and/or Interquartile Range. BMC Med Res. Methodol. 2014, 14, 135. [CrossRef] [PubMed]

15. Hozo, S.P.; Djulbegovic, B.; Hozo, I. Estimating the Mean and Variance from the Median, Range, and the Size of a Sample. BMC Med Res. Methodol. 2005, 5, 13. [CrossRef] [PubMed]

16. Higgins, J.P.T.; Thompson, S.G. Quantifying Heterogeneity in a Meta-Analysis. Stat. Med. 2002, 21, 1539-1558. [CrossRef]

17. National Heart Lung and Blood Institute Quality Assessment Tool for Observational Cohort and Cross-Sectional Studies; National Institutes of Health, Department of Health and Human Services: Bethesda, MD, USA, 2014.

18. Farrah, K.; Young, K.; Tunis, M.C.; Zhao, L. Risk of Bias Tools in Systematic Reviews of Health Interventions: An Analysis of PROSPERO-Registered Protocols. Syst. Rev. 2019, 8, 280. [CrossRef]

19. Akalin, E.; Azzi, Y.; Bartash, R.; Seethamraju, H.; Parides, M.; Hemmige, V.; Ross, M.; Forest, S.; Goldstein, Y.D.; Ajaimy, M.; et al. COVID -19 and Kidney Transplantation. N. Engl. J. Med. 2020, 382, 2475-2477. [CrossRef]

20. Bossini, N.; Alberici, F.; Delbarba, E.; Valerio, F.; Manenti, C.; Possenti, S.; Econimo, L.; Maffei, C.; Pola, A.; Terlizzi, V.; et al. Kidney Transplant Patients with SARS-CoV-2 Infection: The Brescia Renal COVID Task Force Experience. Am. J. Transplant. 2020, 20, 3019-3029. [CrossRef]

21. Trujillo, H.; Caravaca-Fontán, F.; Sevillano, Á.; Gutiérrez, E.; Caro, J.; Gutiérrez, E.; Yuste, C.; Andrés, A.; Praga, M. SARS-CoV-2 Infection in Hospitalized Patients with Kidney Disease. Kidney Int. Rep. 2020, 5, 905-909. [CrossRef]

22. Mohan, S. Early Description of Coronavirus 2019 Disease in Kidney Transplant Recipients in New York. J. Am. Soc. Nephrol. 2020, 31, 1150-1156. [CrossRef]

23. Nair, V.; Jandovitz, N.; Hirsch, J.S.; Nair, G.; Abate, M.; Bhaskaran, M.; Grodstein, E.; Berlinrut, I.; Hirschwerk, D.; Cohen, S.L.; et al. COVID-19 in Kidney Transplant Recipients. Am. J. Transplant. 2020, 20, 1819-1825. [CrossRef]

24. Fernández-Ruiz, M.; Andrés, A.; Loinaz, C.; Delgado, J.F.; López-Medrano, F.; San Juan, R.; González, E.; Polanco, N.; Folgueira, M.D.; Lalueza, A.; et al. COVID-19 in Solid Organ Transplant Recipients: A Single-center Case Series from Spain. Am. J. Transplant. 2020, 20, 1849-1858. [CrossRef] 
25. Zhu, L.; Gong, N.; Liu, B.; Lu, X.; Chen, D.; Chen, S.; Shu, H.; Ma, K.; Xu, X.; Guo, Z.; et al. Coronavirus Disease 2019 Pneumonia in Immunosuppressed Renal Transplant Recipients: A Summary of 10 Confirmed Cases in Wuhan, China. Eur. Urol. 2020, 77, 748-754. [CrossRef] [PubMed]

26. Cavagna, L.; Bruno, R.; Zanframundo, G.; Gregorini, M.; Seminari, E.; Di Matteo, A.; Rampino, T.; Montecucco, C.; Pelenghi, S.; Cattadori, B.; et al. Clinical Presentation and Evolution of COVID-19 in Immunosuppressed Patients. Preliminary Evaluation in a North Italian Cohort on Calcineurin-Inhibitors Based Therapy. medRxiv 2020. [CrossRef]

27. Fung, M.; Chiu, C.Y.; DeVoe, C.; Doernberg, S.B.; Schwartz, B.S.; Langelier, C.; Henrich, T.J.; Yokoe, D.; Davis, J.; Hays, S.R.; et al. Clinical Outcomes and Serologic Response in Solid Organ Transplant Recipients with COVID-19: A Case Series from the United States. Am. J. Transplant. 2020, 20, 3225-3233. [CrossRef]

28. Abrishami, A.; Samavat, S.; Behnam, B.; Arab-Ahmadi, M.; Nafar, M.; Sanei Taheri, M. Clinical Course, Imaging Features, and Outcomes of COVID-19 in Kidney Transplant Recipients. Eur. Urol. 2020, 78, 281-286. [CrossRef]

29. Hoek, R.A.S.; Manintveld, O.C.; Betjes, M.G.H.; Hellemons, M.E.; Seghers, L.; Van Kampen, J.A.A.; Caliskan, K.; Wetering, J.; Hoogen, M.; Metselaar, H.J.; et al. COVID-19 in Solid Organ Transplant Recipients: A Single-center Experience. Transpl. Int. 2020, 33, 1099-1105. [CrossRef] [PubMed]

30. Mella, A.; Mingozzi, S.; Gallo, E.; Lavacca, A.; Rossetti, M.; Clari, R.; Randone, O.; Maffei, S.; Salomone, M.; Imperiale, D.; et al. Case Series of Six Kidney Transplanted Patients with COVID-19 Pneumonia Treated with Tocilizumab. Transpl. Infect. Dis. 2020, 22, e13348. [CrossRef] [PubMed]

31. Montagud-Marrahi, E.; Cofan, F.; Torregrosa, J.; Cucchiari, D.; Ventura-Aguiar, P.; Revuelta, I.; Bodro, M.; Piñeiro, G.J.; Esforzado, N.; Ugalde, J.; et al. Preliminary Data on Outcomes of SARS-CoV-2 Infection in a Spanish Single Center Cohort of Kidney Recipients. Am. J. Transplant. 2020, 20, 2958-2959. [CrossRef]

32. Banerjee, D.; Popoola, J.; Shah, S.; Ster, I.C.; Quan, V.; Phanish, M. COVID-19 Infection in Kidney Transplant Recipients. Kidney Int. 2020, 97, 1076-1082. [CrossRef]

33. Malberti, F.; Pecchini, P.; Marchi, G.; Foramitti, M. When a Nephrology Ward Becomes a COVID-19 Ward: The Cremona Experience. J. Nephrol. 2020, 33, 625-628. [CrossRef] [PubMed]

34. Lubetzky, M.; Aull, M.; Craig-Shapiro, R.; Lee, J.; Lee, J.; Sultan, S.; Marku-Podvorica, J.; Gingras, L.; Kodiyanplakkal, R.P.; Hartono, C.; et al. Kidney Allograft Recipients Diagnosed with Coronavirus Disease-2019: A Single Center Report. medRxiv 2020. [CrossRef]

35. Devresse, A.; Belkhir, L.; Vo, B.; Ghaye, B.; Scohy, A.; Kabamba, B.; Goffin, E.; De Greef, J.; Mourad, M.; De Meyer, M.; et al. COVID-19 Infection in Kidney Transplant Recipients: A Single-Center Case Series of 22 Cases From Belgium. Kidney Med. 2020, 2, 459-466. [CrossRef] [PubMed]

36. Crespo, M.; Pérez-Sáez, M.J.; Redondo-Pachón, D.; Llinàs-Mallol, L.; Montero, M.M.; Villar-García, J.; Arias-Cabrales, C.; Buxeda, A.; Burballa, C.; Vázquez, S.; et al. COVID-19 in Elderly Kidney Transplant Recipients. Am. J. Transplant. 2020, 20, 2883-2889. [CrossRef] [PubMed]

37. Favà, A.; Montero, N.; Cucchiari, D.; Toapanta, N.; Centellas, J.; Vila-Santandreu, A.; Coloma, A.; Meneghini, M.; Manonelles, A.; Sellarès, J.; et al. SARS-CoV-2 in Kidney Transplant Recipients: A Multicentric Prospective Cohort Study. SSRN Electron. J. 2020. Available online: https:/ / ssrn.com/abstract=3594582 (accessed on 10 August 2021). [CrossRef]

38. Zhang, H.; Chen, Y.; Yuan, Q.; Xia, Q.-X.; Zeng, X.-P.; Peng, J.-T.; Liu, J.; Xiao, X.-Y.; Jiang, G.-S.; Xiao, H.-Y.; et al. Identification of Kidney Transplant Recipients with Coronavirus Disease 2019. Eur. Urol. 2020, 77, 742-747. [CrossRef]

39. Cravedi, P.; Mothi, S.S.; Azzi, Y.; Haverly, M.; Farouk, S.S.; Pérez-Sáez, M.J.; Redondo-Pachón, M.D.; Murphy, B.; Florman, S.; Cyrino, L.G.; et al. COVID-19 and Kidney Transplantation: Results from the TANGO International Transplant Consortium. Am. J. Transplant. 2020, 20, 3140-3148. [CrossRef]

40. Maritati, F.; Cerutti, E.; Zuccatosta, L.; Fiorentini, A.; Finale, C.; Ficosecco, M.; Cristiano, F.; Capestro, A.; Balestra, E.; Taruscia, D.; et al. SARS-CoV-2 Infection in Kidney Transplant Recipients: Experience of the Italian Marche Region. Transpl. Infect. Dis. 2020, 22, e13377. [CrossRef]

41. Elias, M.; Pievani, D.; Randoux, C.; Louis, K.; Denis, B.; Delion, A.; Le Goff, O.; Antoine, C.; Greze, C.; Pillebout, E.; et al. COVID-19 Infection in Kidney Transplant Recipients: Disease Incidence and Clinical Outcomes. J. Am. Soc. Nephrol. 2020, 31, 2413-2423. [CrossRef]

42. Chen, T.Y.; Farghaly, S.; Cham, S.; Tatem, L.L.; Sin, J.H.; Rauda, R.; Ribisi, M.; Sumrani, N. COVID-19 Pneumonia in Kidney Transplant Recipients: Focus on Immunosuppression Management. Transpl. Infect. Dis. 2020, 22, e13378. [CrossRef]

43. Mehta, S.A.; Leonard, J.; Labella, P.; Cartiera, K.; Soomro, I.; Neumann, H.; Montgomery, R.A.; Ali, N.M. Outpatient Management of Kidney Transplant Recipients with Suspected COVID-19-Single-center Experience during the New York City Surge. Transpl. Infect. Dis. 2020, 22, e13383. [CrossRef]

44. Silva, F.; Cipriano, A.; Cruz, H.; Tavares, J.; Fragoso, J.; Malheiro, J.; Almeida, M.; Martins, L.S.; Abreu, M.; Pedroso, S.; et al. SARS-CoV-2 Infection in Kidney Transplant Recipients: Early Report of Five Cases. Transpl. Infect. Dis. 2021,23, e13394. [CrossRef]

45. Chavarot, N.; Gueguen, J.; Bonnet, G.; Jdidou, M.; Trimaille, A.; Burger, C.; Amrouche, L.; Weizman, O.; Pommier, T.; Aubert, O.; et al. COVID-19 Severity in Kidney Transplant Recipients Is Similar to Nontransplant Patients with Similar Comorbidities. Am. J. Transplant. 2021, 21, 1285-1294. [CrossRef] 
46. De Meester, J.; De Bacquer, D.; Naesens, M.; Meijers, B.; Couttenye, M.M.; De Vriese, A.S. Incidence, Characteristics, and Outcome of COVID-19 in Adults on Kidney Replacement Therapy: A Regionwide Registry Study. J. Am. Soc. Nephrol. 2021, 32, 385-396. [CrossRef]

47. Abolghasemi, S.; Mardani, M.; Sali, S.; Honarvar, N.; Baziboroun, M. COVID-19 and Kidney Transplant Recipients. Transpl. Infect. Dis. 2020, 22, e13413. [CrossRef]

48. Cucchiari, D.; Guillén, E.; Cofan, F.; Torregrosa, J.; Esforzado, N.; Revuelta, I.; Ventura-Aguiar, P.; Oppenheimer, F.; Bayés, B.; Marcos, M.Á.; et al. Taking Care of Kidney Transplant Recipients during the COVID-19 Pandemic: Experience from a Medicalized Hotel. Clin. Transplant. 2021, 35, e14132. [CrossRef] [PubMed]

49. Kute, V.B.; Bhalla, A.K.; Guleria, S.; Ray, D.S.; Bahadur, M.M.; Shingare, A.; Hegde, U.; Gang, S.; Raju, S.; Patel, H.V.; et al. Clinical Profile and Outcome of COVID-19 in 250 Kidney Transplant Recipients: A Multicenter Cohort Study From India. Transplantation 2021, 105, 851-860. [CrossRef] [PubMed]

50. Caillard, S.; Anglicheau, D.; Matignon, M.; Durrbach, A.; Greze, C.; Frimat, L.; Thaunat, O.; Legris, T.; Moal, V.; Westeel, P.F.; et al. An Initial Report from the French SOT COVID Registry Suggests High Mortality Due to COVID-19 in Recipients of Kidney Transplants. Kidney Int. 2020, 98, 1549-1558. [CrossRef] [PubMed]

51. Kutzler, H.L.; Poulos, C.M.; Cheema, F.; O'Sullivan, D.M.; Ali, A.; Ebcioglu, Z.; Einstein, M.; Feingold, A.D.; Gluck, J.; Hammond, J.A.; et al. COVID-19 in Solid Organ Transplant Recipients: Observations From Connecticut. Transplantation 2021, 105, e6-e8. [CrossRef] [PubMed]

52. Miarons, M.; Larrosa-García, M.; García-García, S.; Los-Arcos, I.; Moreso, F.; Berastegui, C.; Castells, L.; Pérez-Hoyos, S.; Varela, J.; Pau-Parra, A.; et al. COVID-19 in Solid Organ Transplantation: A Matched Retrospective Cohort Study and Evaluation of Immunosuppression Management. Transplantation 2021, 105, 138-150. [CrossRef]

53. Sharma, P.; Chen, V.; Fung, C.M.; Troost, J.P.; Patel, V.N.; Combs, M.; Norman, S.; Garg, P.; Colvin, M.; Aaronson, K.; et al. COVID-19 Outcomes Among Solid Organ Transplant Recipients: A Case-Control Study. Transplantation 2021, 105, 128-137. [CrossRef]

54. Mohamed, I.H.; Chowdary, P.B.; Shetty, S.; Sammartino, C.; Sivaprakasam, R.; Lindsey, B.; Thuraisingham, R.; Yaqoob, M.M.; Khurram, M.A. Outcomes of Renal Transplant Recipients with SARS-CoV-2 Infection in the Eye of the Storm: A Comparative Study with Waitlisted Patients. Transplantation 2021, 105, 115-120. [CrossRef]

55. Felldin, M.; Søfteland, J.M.; Magnusson, J.; Ekberg, J.; Karason, K.; Schult, A.; Larsson, H.; Oltean, M.; Friman, V. Initial Report From a Swedish High-Volume Transplant Center After the First Wave of the COVID-19 Pandemic. Transplantation 2021, 105, 108-114. [CrossRef]

56. Kates, O.S.; Haydel, B.M.; Florman, S.S.; Rana, M.M.; Chaudhry, Z.S.; Ramesh, M.S.; Safa, K.; Kotton, C.N.; Blumberg, E.A.; Besharatian, B.D.; et al. Coronavirus Disease 2019 in Solid Organ Transplant: A Multicenter Cohort Study. Clin. Infect. Dis. 2020, ciaa1097. [CrossRef] [PubMed]

57. Benotmane, I.; Gautier-Vargas, G.; Wendling, M.; Perrin, P.; Velay, A.; Bassand, X.; Bedo, D.; Baldacini, C.; Sagnard, M.; Bozman, D.; et al. In-depth Virological Assessment of Kidney Transplant Recipients with COVID-19. Am. J. Transplant. 2020, 20, 3162-3172. [CrossRef] [PubMed]

58. Chaudhry, Z.S.; Williams, J.D.; Vahia, A.; Fadel, R.; Parraga Acosta, T.; Prashar, R.; Shrivastava, P.; Khoury, N.; Pinto Corrales, J.; Williams, C.; et al. Clinical Characteristics and Outcomes of COVID-19 in Solid Organ Transplant Recipients: A Cohort Study. Am. J. Transplant. 2020, 20, 3051-3060. [CrossRef] [PubMed]

59. Craig-Schapiro, R.; Salinas, T.; Lubetzky, M.; Abel, B.T.; Sultan, S.; Lee, J.R.; Kapur, S.; Aull, M.J.; Dadhania, D.M. COVID-19 Outcomes in Patients Waitlisted for Kidney Transplantation and Kidney Transplant Recipients. Am. J. Transplant. 2021, 21, 1576-1585. [CrossRef]

60. Demir, E.; Uyar, M.; Parmaksiz, E.; Sinangil, A.; Yelken, B.; Dirim, A.B.; Merhametsiz, O.; Yadigar, S.; Atan Ucar, Z.; Ucar, A.R.; et al. COVID-19 in Kidney Transplant Recipients: A Multicenter Experience in Istanbul. Transpl. Infect. Dis. 2020, 22, e13371. [CrossRef] [PubMed]

61. Hilbrands, L.B.; Duivenvoorden, R.; Vart, P.; Franssen, C.F.M.; Hemmelder, M.H.; Jager, K.J.; Kieneker, L.M.; Noordzij, M.; Pena, M.J.; de Vries, H.; et al. COVID-19-Related Mortality in Kidney Transplant and Dialysis Patients: Results of the ERACODA Collaboration. Nephrol. Dial. Transplant. 2020, 35, 1973-1983. [CrossRef] [PubMed]

62. Pascual, J.; Melilli, E.; Jiménez-Martín, C.; González-Monte, E.; Zárraga, S.; Gutiérrez-Dalmau, A.; López-Jiménez, V.; Juega, J.; Muñoz-Cepeda, M.; Lorenzo, I.; et al. COVID-19-Related Mortality During the First 60 Days After Kidney Transplantation. Eur. Urol. 2020, 78, 641-643. [CrossRef] [PubMed]

63. Rodriguez-Cubillo, B.; Higuera, M.A.M.; Lucena, R.; Franci, E.V.; Hurtado, M.; Romero, N.C.; Moreno, A.R.; Valencia, D.; Velo, M.; Fornie, I.S.; et al. Should Cyclosporine Be Useful in Renal Transplant Recipients Affected by SARS-CoV-2? Am. J. Transplant. 2020, 20, 3173-3181. [CrossRef] [PubMed]

64. Sánchez-Alvarez, E.; Macía, M.; de Sequera Ortiz, P. Management of Hemodialysis Patients with Suspected or Confirmed COVID-19 Infection: Perspective from the Spanish Nephrology. Kidney360 2020, 1, 1254-1258. [CrossRef]

65. Oto, O.A.; Ozturk, S.; Turgutalp, K.; Arici, M.; Alpay, N.; Merhametsiz, O.; Sipahi, S.; Ogutmen, M.B.; Yelken, B.; Altiparmak, M.R.; et al. Predicting the Outcome of COVID-19 Infection in Kidney Transplant Recipients. BMC Nephrol. 2021, 22, 100. [CrossRef] [PubMed] 
66. Elhadedy, M.A.; Marie, Y.; Halawa, A. COVID-19 in Renal Transplant Recipients: Case Series and a Brief Review of Current Evidence. Nephron 2020, 145, 192-198. [CrossRef]

67. Zeng, F.; Huang, Y.; Guo, Y.; Yin, M.; Chen, X.; Xiao, L.; Deng, G. Association of Inflammatory Markers with the Severity of COVID-19: A Meta-Analysis. Int. J. Infect. Dis. 2020, 96, 467-474. [CrossRef]

68. Schnuelle, P.; Lorenz, D.; Trede, M.; Van Der Woude, F.J. Impact of Renal Cadaveric Transplantation on Survival in End-Stage Renal Failure: Evidence for Reduced Mortality Risk Compared with Hemodialysis during Long-Term Follow-Up. J. Am. Soc. Nephrol. 1998, 9, 2135-2141. [CrossRef]

69. Port, F.K.; Wolfe, R.A.; Mauger, E.A.; Berling, D.P.; Jiang, K. Comparison of Survival Probabilities for Dialysis Patients vs Cadaveric Renal Transplant Recipients. JAMA: J. Am. Med Assoc. 1993, 270, 1339-1343. [CrossRef]

70. Ojo, A.O.; Port, F.K.; Wolfe, R.A.; Mauger, E.A.; Williams, L.; Berling, D.P. Comparative Mortality Risks of Chronic Dialysis and Cadaveric Transplantation in Black End-Stage Renal Disease Patients. Am. J. Kidney Dis. 1994, 24, 59-64. [CrossRef]

71. Su, H.; Yang, M.; Wan, C.; Yi, L.-X.; Tang, F.; Zhu, H.-Y.; Yi, F.; Yang, H.-C.; Fogo, A.B.; Nie, X.; et al. Renal Histopathological Analysis of 26 Postmortem Findings of Patients with COVID-19 in China. Kidney Int. 2020, 98, 219-227. [CrossRef]

72. Larsen, C.P.; Bourne, T.D.; Wilson, J.D.; Saqqa, O.; Sharshir, M.A. Collapsing Glomerulopathy in a Patient with Coronavirus Disease 2019 (COVID-19). Kidney Int. Rep. 2020, 5, 935. [CrossRef] [PubMed]

73. Varga, Z.; Flammer, A.J.; Steiger, P.; Haberecker, M.; Andermatt, R.; Zinkernagel, A.S.; Mehra, M.R.; Schuepbach, R.A.; Ruschitzka, F.; Moch, H. Endothelial Cell Infection and Endotheliitis in COVID-19. Lancet 2020, 395, 1417-1418. [CrossRef]

74. Ronco, C.; Reis, T. Kidney Involvement in COVID-19 and Rationale for Extracorporeal Therapies. Nat. Rev. Nephrol. 2020, 16, 308-310. [CrossRef]

75. Zhang, Y.; Xiao, M.; Zhang, S.; Xia, P.; Cao, W.; Jiang, W.; Chen, H.; Ding, X.; Zhao, H.; Zhang, H.; et al. Coagulopathy and Antiphospholipid Antibodies in Patients with COVID -19. N. Engl. J. Med. 2020, 382, e38. [CrossRef] [PubMed]

76. Report of the WHO-China Joint Mission on Coronavirus Disease 2019 (COVID-19). 16-24 February 2020. Available online: https:/ / www.who.int/docs / default-source/coronaviruse/who-china-joint-mission-on-covid-19-final-report.pdf (accessed on 10 August 2021).

77. Yang, J.; Zheng, Y.; Gou, X.; Pu, K.; Chen, Z.; Guo, Q.; Ji, R.; Wang, H.; Wang, Y.; Zhou, Y. Prevalence of Comorbidities and Its Effects in Patients Infected with SARS-CoV-2: A Systematic Review and Meta-Analysis. Int. J. Infect. Dis. 2020, 94, 91-95. [CrossRef]

78. Chen, T.; Wu, D.; Chen, H.; Yan, W.; Yang, D.; Chen, G.; Ma, K.; Xu, D.; Yu, H.; Wang, H.; et al. Clinical Characteristics of 113 Deceased Patients with Coronavirus Disease 2019: Retrospective Study. BMJ 2020, 368, m1091. [CrossRef]

79. Li, X.; Xu, S.; Yu, M.; Wang, K.; Tao, Y.; Zhou, Y.; Shi, J.; Zhou, M.; Wu, B.; Yang, Z.; et al. Risk Factors for Severity and Mortality in Adult COVID-19 Inpatients in Wuhan. J. Allergy Clin. Immunol. 2020, 146, 110-118. [CrossRef]

80. Guan, W.J.; Liang, W.H.; Zhao, Y.; Liang, H.R.; Chen, Z.S.; Li, Y.M.; Liu, X.Q.; Chen, R.C.; Tang, C.L.; Wang, T.; et al. Comorbidity and Its Impact on 1,590 Patients with COVID -19 in China: A Nationwide Analysis. Eur. Respir. J. 2020, 55, 200054. [CrossRef]

81. Robbins-Juarez, S.Y.; Qian, L.; King, K.L.; Stevens, J.S.; Husain, S.A.; Radhakrishnan, J.; Mohan, S. Outcomes for Patients with COVID-19 and Acute Kidney Injury: A Systematic Review and Meta-Analysis. Kidney Int. Rep. 2020, 5, 1149-1160. [CrossRef]

82. Pereira, M.R.; Mohan, S.; Cohen, D.J.; Husain, S.A.; Dube, G.K.; Ratner, L.E.; Arcasoy, S.; Aversa, M.M.; Benvenuto, L.J.; Dadhania, D.M.; et al. COVID-19 in Solid Organ Transplant Recipients: Initial Report from the US Epicenter. Am. J. Transplant. 2020, 20, 1800-1808. [CrossRef]

83. Azzi, Y.; Parides, M.; Alani, O.; Loarte-Campos, P.; Bartash, R.; Forest, S.; Colovai, A.; Ajaimy, M.; Liriano-Ward, L.; Pynadath, C.; et al. COVID-19 Infection in Kidney Transplant Recipients at the Epicenter of Pandemics. Kidney Int. 2020, 98, 1559-1567. [CrossRef] [PubMed]

84. Yang, X.; Yu, Y.; Xu, J.; Shu, H.; Xia, J.; Liu, H.; Wu, Y.; Zhang, L.; Yu, Z.; Fang, M.; et al. Clinical Course and Outcomes of Critically Ill Patients with SARS-CoV-2 Pneumonia in Wuhan, China: A Single-Centered, Retrospective, Observational Study. Lancet Respir. Med. 2020, 8, 475-481. [CrossRef]

85. Wu, C.; Chen, X.; Cai, Y.; Xia, J.; Zhou, X.; Xu, S.; Huang, H.; Zhang, L.; Zhou, X.; Du, C.; et al. Risk Factors Associated with Acute Respiratory Distress Syndrome and Death in Patients with Coronavirus Disease 2019 Pneumonia in Wuhan, China. JAMA Intern. Med. 2020, 180, 934. [CrossRef] [PubMed]

86. Arentz, M.; Yim, E.; Klaff, L.; Lokhandwala, S.; Riedo, F.X.; Chong, M.; Lee, M. Characteristics and Outcomes of 21 Critically Ill Patients with COVID-19 in Washington State. JAMA 2020, 323, 1612. [CrossRef]

87. Grasselli, G.; Greco, M.; Zanella, A.; Albano, G.; Antonelli, M.; Bellani, G.; Bonanomi, E.; Cabrini, L.; Carlesso, E.; Castelli, G.; et al. Risk Factors Associated with Mortality Among Patients with COVID-19 in Intensive Care Units in Lombardy, Italy. JAMA Intern. Med. 2020, 180, 1345. [CrossRef]

88. Williamson, E.J.; Walker, A.J.; Bhaskaran, K.; Bacon, S.; Bates, C.; Morton, C.E.; Curtis, H.J.; Mehrkar, A.; Evans, D.; Inglesby, P.; et al. Factors Associated with COVID-19-Related Death Using OpenSAFELY. Nature 2020, 584, 430-436. [CrossRef] [PubMed]

89. Armstrong, R.A.; Kane, A.D.; Cook, T.M. Outcomes from Intensive Care in Patients with COVID-19: A Systematic Review and Meta-Analysis of Observational Studies. Anaesthesia 2020, 75, 1340-1349. [CrossRef] 
90. Sánchez-Álvarez, J.E.; Fontán, M.P.; Martín, C.J.; Pelícano, M.B.; Reina, C.J.C.; Prieto, Á.M.S.; Melilli, E.; Barrios, M.C.; Heras, M.M.; y Pino, M.D. Status of SARS-CoV-2 Infection in Patients on Renal Replacement Therapy. Report of the COVID-19 Registry of the Spanish Society of Nephrology (SEN). Nefrología 2020, 40, 272-278. [CrossRef] [PubMed]

91. Felsenstein, S.; Herbert, J.A.; McNamara, P.S.; Hedrich, C.M. COVID-19: Immunology and Treatment Options. Clin. Immunol. 2020, 215, 108448. [CrossRef]

92. Carsetti, R.; Zaffina, S.; Piano Mortari, E.; Terreri, S.; Corrente, F.; Capponi, C.; Palomba, P.; Mirabella, M.; Cascioli, S.; Palange, P.; et al. Different Innate and Adaptive Immune Responses to SARS-CoV-2 Infection of Asymptomatic, Mild, and Severe Cases. Front. Immunol. 2020, 11, 3365. [CrossRef] [PubMed]

93. Mathew, D.; Giles, J.R.; Baxter, A.E.; Oldridge, D.A.; Greenplate, A.R.; Wu, J.E.; Alanio, C.; Kuri-Cervantes, L.; Pampena, M.B.; D'Andrea, K.; et al. Deep Immune Profiling of COVID-19 Patients Reveals Distinct Immunotypes with Therapeutic Implications. Science 2020, 369, eabc8511. [CrossRef] [PubMed]

94. Wang, F.; Nie, J.; Wang, H.; Zhao, Q.; Xiong, Y.; Deng, L.; Song, S.; Ma, Z.; Mo, P.; Zhang, Y. Characteristics of Peripheral Lymphocyte Subset Alteration in COVID-19 Pneumonia. J. Infect. Dis. 2020, 221, 1762-1769. [CrossRef]

95. Thevarajan, I.; Nguyen, T.H.O.; Koutsakos, M.; Druce, J.; Caly, L.; van de Sandt, C.E.; Jia, X.; Nicholson, S.; Catton, M.; Cowie, B.; et al. Breadth of Concomitant Immune Responses Prior to Patient Recovery: A Case Report of Non-Severe COVID-19. Nat. Med. 2020, 26, 453-455. [CrossRef]

96. Johnston, O.; Jaswal, D.; Gill, J.S.; Doucette, S.; Fergusson, D.A.; Knoll, G.A. Treatment of Polyomavirus Infection in Kidney Transplant Recipients: A Systematic Review. Transplantation 2010, 89, 1057-1070. [CrossRef] [PubMed]

97. Saad, E.R.; Bresnahan, B.A.; Cohen, E.P.; Lu, N.; Orentas, R.J.; Vasudev, B.; Hariharan, S. Successful Treatment of BK Viremia Using Reduction in Immunosuppression without Antiviral Therapy. Transplantation 2008, 85, 850-854. [CrossRef] [PubMed]

98. de Mezerville, M.H.; Tellier, R.; Richardson, S.; Hébert, D.; Doyle, J.; Allen, U. Adenoviral Infections in Pediatric Transplant Recipients. Pediatr. Infect. Dis. J. 2006, 25, 815-818. [CrossRef]

99. Watcharananan, S.P.; Avery, R.; Ingsathit, A.; Malathum, K.; Chantratita, W.; Mavichak, V.; Chalermsanyakorn, P.; Jirasiritham, S.; Sumethkul, V. Adenovirus Disease after Kidney Transplantation: Course of Infection and Outcome in Relation to Blood Viral Load and Immune Recovery. Am. J. Transplant. 2011, 11, 1308-1314. [CrossRef]

100. Mehta, P.; McAuley, D.F.; Brown, M.; Sanchez, E.; Tattersall, R.S.; Manson, J.J. COVID-19: Consider Cytokine Storm Syndromes and Immunosuppression. Lancet 2020, 395, 1033-1034. [CrossRef]

101. Ritchie, A.I.; Singanayagam, A. Immunosuppression for Hyperinflammation in COVID-19: A Double-Edged Sword? Lancet 2020, 395, 1111. [CrossRef]

102. Ye, Z.; Wang, Y.; Colunga-Lozano, L.E.; Prasad, M.; Tangamornsuksan, W.; Rochwerg, B.; Yao, L.; Motaghi, S.; Couban, R.J.; Ghadimi, M.; et al. Efficacy and Safety of Corticosteroids in COVID-19 Based on Evidence for COVID-19, Other Coronavirus Infections, Influenza, Community-Acquired Pneumonia and Acute Respiratory Distress Syndrome: A Systematic Review and Meta-Analysis. Can. Med. Assoc. J. 2020, 192, E756-E767. [CrossRef]

103. Horby, P.; Lim, W.S.; Emberson, J.R.; Mafham, M.; Bell, J.L.; Linsell, L.; Staplin, N.; Brightling, C.; Ustianowski, A.; Elmahi, E.; et al. Dexamethasone in Hospitalized Patients with COVID -19_Preliminary Report. N. Engl. J. Med. 2021, 384, 693-704. [CrossRef] [PubMed] 